BULLETIN (New Series) OF THE

AMERICAN MATHEMATICAL SOCIETY

Volume 39, Number 2, Pages 207-265

S 0273-0979(02)00929-1

Article electronically published on January 4, 2002

\title{
SELECTED NEW ASPECTS OF THE CALCULUS OF VARIATIONS IN THE LARGE
}

\author{
IVAR EKELAND AND NASSIF GHOUSSOUB
}

\begin{abstract}
We discuss some of the recent developments in variational methods while emphasizing new applications to nonlinear problems. We touch on several issues: (i) the formulation of variational set-ups which provide more information on the location of critical points and therefore on the qualitative properties of the solutions of corresponding Euler-Lagrange equations; (ii) the relationships between the energy of variationally generated solutions, their Morse indices, and the Hausdorff measure of their nodal sets; (iii) the gluing of several topological obstructions; (iv) the preservation of critical levels after deformation of functionals; (v) and the various ways to recover compactness in certain borderline variational problems.
\end{abstract}

\section{Contents}

1. Introduction

2. Critical points and non-linear variational problems

2.1. Variational proofs of non-existence: Pohozaev phenomenon 210

2.2. The min-max identification of critical levels

2.3. Analysis of Palais-Smale sequences in borderline variational problems

3. Locating critical points via dual sets

3.1. Improved compactness around dual sets 223

3.2. Relaxing boundary conditions in the presence of dual sets 225

4. Duality and the multiplicity of critical points 227

4.1. Group actions and topological indices $\quad 227$

4.2. Multiplicity results à la Ljusternik-Schnirelmann 228

4.3. Comparable functions and other types of multiplicity $\quad 230$

5. Morse indices of variationally generated critical points 231

5.1. Distinguishing solutions via their Morse indices 233

5.2. Morse indices and energy estimates 234

5.3. Morse indices, nodal sets and $L^{\infty}$-estimates 235

6. Creating and preserving topological obstructions 237

6.1. Preserving min-max levels after deformation of symmetry 237

6.2. Multiplicity results for non-homogeneous equations 239

6.3. Tensor products of min-max classes 241

Received by the editors January 2001 and, in revised form, June 13, 2001.

2000 Mathematics Subject Classification. Primary 35J60, 47J30, 58E05; Secondary 57R17.

The second author was partially supported by a grant from the Natural Science and Engineering Research Council of Canada (NSERC). 
6.4. Multi-peak solutions for singularly perturbed problems 243

7. Parameter dependent functionals and compactness 245

7.1. Constructing bounded Palais-Smale sequences 245

7.2. The superquadraticity condition 247

8. Recovering compactness from second order information 249

8.1. Variational principles with second order information 250

8.2. Compactness via upper estimates on approximate Morse indices 250

9. Towards a $C^{0}$-theory of critical points 253

9.1. Structure of the critical set in the mountain pass theorem 255

\begin{tabular}{lll}
9.2 . Classification of higher dimensional saddle points & 258 \\
\hline
\end{tabular}

10. Elements of symplectic topology 259

11. By way of conclusion $\quad 261$

\begin{tabular}{ll} 
References & 262 \\
\hline
\end{tabular}

\section{INTRODUCTION}

The calculus of variations has over and over again proved itself to be one of the most powerful and far-reaching tools available for advancing our understanding of mathematics and its applications. This is no doubt due to the fact that variational methods are not merely techniques for solving individual, albeit very important, problems but are often "variational principles"; i.e. they are manifestations of very general laws of nature which are valid in diverse branches of physics, biology, economics and engineering.

Finding critical levels of a functional by minimization is as ancient as the least action principle of Fermat and Maupertuis, and the calculus of variations has been an active field of mathematics for almost three centuries. This will not be our focus here as we try to deal with indefinite functionals and with issues of multiplicity of solutions, hence with the existence of unstable extrema.

The modern variational approach to non-linear problems was initiated by G. Birkhoff in 1917. His min-max methods were quickly subsumed by the striking inequalities established by his student, M. Morse, who revealed the deep relationship between the number and types of critical points of functionals and the topology of their domains of definition. Soon after, Birkhoff established that Morse's inequalities actually follow from his min-max methods. Around the same time, this approach was also being developed and used by Ljusternik and Schnirelmann to establish the existence of 3 distinct closed geodesics on any compact surface of genus zero. These methods and results - which also mark the beginning of global analysis - were finite dimensional in nature. The development of infinite dimensional tools accelerated in the 1960's. They are currently being actively refined and extended so as to overcome the limitations to their applicability to the theory of partial differential equations: limitations induced by the prohibitive compactness, non-degeneracy and regularity conditions that are often not satisfied by present-day variational problems.

The subject has come of age in the last thirty years, and a number of surveys and monographs have described much of the progress [E2], [N], R1], [M-W], St], Ch], G2]. In the present paper, we discuss some of the more recent developments of 
these variational methods while emphasizing new applications to non-linear problems. We touch on several issues: (i) the formulation of variational set-ups which provide more information on the location and therefore on the qualitative properties of critical points (Ghoussoub-Preiss duality methods); (ii) the relationships between the energy of variationally generated solutions, their Morse indices, and the Hausdorff measure of their nodal sets (Bahri-Lions and Yang's estimates); (iii) the identification of new topological obstructions (Séré's tensor products); (vi) the preservation of critical levels after deformation of functionals (Bolle's principle); (v) the recovery of compactness in borderline variational problems (Struwe's monotonicity trick, restricted compactness around dual sets, Taubes' and P.L. Lions' use of a-priori estimates on Morse indices).

Each "general principle" is illustrated by one or more examples. These were chosen to illustrate the variety of applications: semilinear elliptic PDEs, Hamiltonian systems and symplectic topology, second order systems, closed geodesic problems and Hartree-Fock equations. We have also tried to make the paper accessible to a general audience while still offering a few novelties to the specialists.

The rapid development of this area and the variety of applications forced us to be quite selective. We mostly concentrate on certain advances not covered in Struwe's monograph - mentioned above- which at present seems to be the authoritative reference on the subject. Our choices reflect our taste and what we know, of course, but also our perceptions of the novel methods and ideas, those that are minimally ad-hoc and not particular to any specific problem, as well as those we consider potentially applicable in a wide range of problems. However, this exposition is still far from being a complete picture of this fast developing field. Indeed, we do not address the approaches of Conley and Floer to Morse theory [Co1], [F1, the machinery involving critical points at infinity developed by A. Bahri and his collaborators [Ba] or the fundamental contributions of C. Taubes Tau1 to infinite

dimensional Morse theory in the context of the Yang-Mills equations. The reason for these omissions is simply that these theories, which are substantialy more involved than the elementary approach adopted here, deserve nothing less but their own comprehensive surveys. We have greatly benefited, during the preparation of this report, from the input, criticism and high standards of our good friends and colleagues Changfeng Gui and Eric Séré. To them we are grateful.

\section{CRitical POINTS AND NON-LINEAR VARIATIONAL PROBLEMS}

Non-linear problems such as those that naturally arise in the study of geodesics, minimal surfaces, harmonic maps, conformal metrics with prescribed curvature, subharmonics of Hamiltonian systems, solutions of boundary value problems and Yang-Mills fields can all be characterized as critical points $u$ of some functional $I$ on an appropriate manifold $X$, i.e., $I^{\prime}(u)=0$. We will be concerned with problems of existence, location, multiplicity and qualitative properties of critical points in such contexts and how they relate to the (weak) solutions they represent for the corresponding Euler-Lagrange equations.

Our first illustration of the variational methods deals with the following type of boundary value problems:

$$
\left\{\begin{aligned}
-\Delta_{p} u & =\lambda|u|^{r-2} u+\mu \frac{|u|^{q-2}}{|x|^{s}} u & & \text { in } \Omega \\
u & =0 & & \text { on } \partial \Omega,
\end{aligned}\right.
$$


where $\lambda$ and $\mu$ are two positive parameters and $\Omega$ is a smooth bounded domain in $\mathbf{R}^{n}$ containing 0 . Recall that $\Delta_{p} u=\nabla \cdot\left(|\nabla u|^{p-2} \nabla u\right)$. Assume that $0 \leq s \leq p<n$.

The starting point of the variational approach to these problems is the following inequality which can be obtained by essentially "interpolating" between Sobolev's and Hardy's inequalities [C-K-N]. Assume that $1<p<n$ and $p \leq q \leq p^{*}(s) \equiv$ $\frac{n-s}{n-p} p$; then there is a constant $C>0$ such that:

$$
C\left(\int_{\Omega} \frac{|u|^{q}}{|x|^{s}} d x\right)^{\frac{p}{q}} \leq \int_{\Omega}|\nabla u|^{p} d x \text { for all } u \in H_{0}^{1, p}(\Omega)
$$

By assuming that $p \leq r \leq p^{*} \equiv p^{*}(0)=\frac{n p}{n-p}$ for the non-singular term, we can guarantee that the functional

$$
I_{\lambda, \mu}(u):=\frac{1}{p} \int_{\Omega}|\nabla u|^{p} d x-\frac{\lambda}{r} \int_{\Omega}|u|^{r} d x-\frac{\mu}{q} \int_{\Omega} \frac{|u|^{q}}{|x|^{s}} d x
$$

is well defined on the Sobolev space $H_{0}^{1, p}(\Omega)$. The (weak) solutions of problem (2.1) are then the critical points of the functional $I_{\lambda, \mu}$. Here are a few facts:

The map $u \rightarrow \frac{u}{x^{s / q}}$ from $H_{0}^{p}(\Omega)$ into $L^{q}(\Omega)$ is compact provided $q<p^{*}(s)$. The best Sobolev-Hardy constant is defined as:

$$
\mu_{s, q}(\Omega):=\inf \left\{\int_{\Omega}|\nabla u|^{p} d x ; u \in H_{0}^{1, p}(\Omega), \int_{\Omega} \frac{|u|^{q}}{|x|^{s}} d x=1\right\} .
$$

In the important case where $q=p^{*}(s)$, we simply denote $\mu_{s, p^{*}(s)}(\Omega)$ by $\mu_{s}(\Omega)$. Note that $\mu_{0}(\Omega)$ is nothing but the best constant in the Sobolev inequality while $\mu_{p}(\Omega)$ is the best constant in the Hardy inequality, i.e.,

$$
\mu_{p}(\Omega):=\inf \left\{\int_{\Omega}|\nabla u|^{p} d x ; u \in H_{0}^{1, p}(\Omega), \int_{\Omega} \frac{|u|^{p}}{|x|^{p}} d x=1\right\} .
$$

Another relevant parameter is the first "eigenvalue" of the p-Laplacian $-\Delta_{p}$ defined as

$$
\lambda_{1}(\Omega):=\mu_{0, p}(\Omega)=\inf \left\{\int_{\Omega}|\nabla w|^{p} d x ; w \in H_{0}^{1, p}(\Omega), \int_{\Omega}|w|^{p} d x=1\right\} .
$$

2.1. Variational proofs of non-existence: Pohozaev phenomenon. Our first illustration of modern variational methods deals with non-existence results. In the following example, we describe a simple but far-reaching idea of Pohozaev $[\mathrm{P}]$.

Proposition 2.1. If $\Omega$ is a smooth star-shaped domain in $\mathbf{R}^{n}$ containing 0 , then problem (2.1) has no non-trivial solution in the doubly critical case: i.e., when $r=p^{*}$ and $q=p^{*}(s)=\frac{n-s}{n-p} p$ where $0 \leq s \leq p$.

Indeed, if $v$ is the outward normal to $\partial \Omega$, then $\langle x, v\rangle>0$ on $\partial \Omega$. Multiply equation $(2.1)$ by $\langle x, \nabla u\rangle$ on both sides and integrate by parts to get:

$$
\frac{p-1}{p} \int_{\partial \Omega}|\nabla u|^{p}\langle x, v\rangle d x+\frac{n-p}{p} \int_{\Omega}|\nabla u|^{p} d x=\mu \frac{n-s}{q} \int_{\Omega} \frac{|u|^{q}}{|x|^{s}} d x+\lambda \frac{n}{r} \int_{\Omega}|u|^{r} d x .
$$

On the other hand, multiplying equation (2.1) by $u$ and integrating, we get

$$
\int_{\Omega}|\nabla u|^{p} d x=\mu \int_{\Omega} \frac{|u|^{q}}{|x|^{s}} d x+\lambda \int_{\Omega}|u|^{r} d x
$$


Putting the two identities together, we obtain

$$
\frac{p-1}{p} \int_{\partial \Omega}|\nabla u|^{p}\langle x, v\rangle d \sigma=\mu\left(\frac{n-s}{q}-\frac{n-p}{p}\right) \int_{\Omega} \frac{|u|^{q}}{|x|^{s}} d x+\lambda\left(\frac{n}{r}-\frac{n-p}{p}\right) \int_{\Omega}|u|^{r} d x .
$$

So if $r=\frac{n p}{n-p}=p^{*}$ and $q=\frac{n-s}{n-p} p$, problem (2.1) has no non-trivial solution.

Remark 2.1. What is hidden behind this computation is that Pohozaev-type identities -for a general functional $I$-come out from comparing two different variations of $I$ at a critical point $u$ :

$$
\left\{\begin{array}{c}
\frac{d}{d \epsilon}[I(u(x+\epsilon \nabla \alpha(x)))]_{\epsilon=0}=0 \\
\frac{d}{d \epsilon}[I(u(x)(1+\epsilon \beta(x)))]_{\epsilon=0}=0
\end{array}\right.
$$

where $\alpha$ and $\beta$ are two sufficiently smooth functions on $\mathbf{R}^{n}$. The most obvious candidates (which correspond to the example above) are $\alpha(x)=\frac{1}{2}|x|^{2}$, which can be seen as a derivative with respect to scaling, and $\beta(x)=1$. Other choices for $\nabla \alpha$ (or a more general vector field) and $\beta$ can also lead to other useful identities. It is an instructive exercise to generate new identities -using other functions (or even vector fields) $\alpha$ and $\beta$ - for a functional of the form $I(u)=\int_{\mathbf{R}^{n}}|\nabla u|^{2} d x-\int_{\mathbf{R}^{n}} F(x, u) d x$ whose critical points are (weak) solutions of the equation $-\Delta u=F^{\prime}(x, u)$ in $\mathbf{R}^{n}$.

2.2. The min-max identification of critical levels. Locating critical levels for a smooth functional $I$ on a manifold $X$ essentially reduces to capturing the changes in the topology of the sublevel sets $I^{a}=\{x \in X ; I(x)<a\}$ as $a$ varies in $\mathbf{R}$. Under the right conditions on $I$, classical Morse theory states that a non-trivial relative homology group $H_{*}\left(I^{b}, I^{a}\right)$ should detect a critical level between $a$ and $b$, since otherwise $I^{a}$ will be a deformation retract of $I^{b}$ via the negative gradient flow of $I$. The simplest example -short of considering minimization- consists of taking two points ("villages") $u_{0}$ and $u_{1}$ both lying below level $a$ which are not connected in $I^{a}$, but become so if one can climb above that level. This means that the first homotopy group $\pi_{1}\left(I^{b}, I^{a}\right)$ is non-trivial for some $b>a$ and the first Morse inequality yields a critical point of Morse index 1 between levels $a$ and $b$. This setting is often called the mountain-pass principle since in practice one insures that the two villages are disconnected below level $a$ by showing that they are separated by a "mountain range" $M$ with minimal altitude exceeding $a$. In other words, $\gamma \cap M \neq \emptyset$ for every $\gamma$ in the class $\mathcal{F}_{u_{0}}^{u_{1}}$ of all continuous paths joining $u_{0}$ and $u_{1}$ in $X$, which readily implies that

$$
\max \left\{I\left(u_{0}\right), I\left(u_{1}\right)\right\}<\inf _{M} I \leq c:=\inf _{\gamma \in \mathcal{F}_{u_{0}}^{u_{1}}} \max _{x \in \gamma} I(x) .
$$

Hence the required change in topology occurs between levels $c-\epsilon$ and $c+\epsilon$.

Analysts often use this intuitive and constructive method (and its higher dimensional min-max generalizations) to compute topological obstructions and identify critical levels. We shall adopt this point of view throughout, for many reasons: First, the setting - as in minimization problems - is purely topological, requires hardly any regularity and is amenable to deal with possibly degenerate and nonsmooth functionals in non-compact situations. Secondly, the intersection property mentioned above leads to the important concept of duality between - families of sets and its many applications: one of them being the extraction of purely topological qualitative information on the variationally generated critical points. Thirdly, 
it allows for a global view at the dependence of the critical values on the functionals: for example, variationally generated critical values of $I$ depend monotonically and continuously (in the $C^{0}$ topology) on $I$. In other words, the min-max classes contain all the information and seem to be as important and useful as the critical points they would generate in the presence of compactness.

Now some notation and terminology: A critical point of $I$ is a point where $I^{\prime}(x)=0$. A critical value of $I$ is a number $c$ such that $I(x)=c$ for some critical point $x$. An approximate critical (or Palais-Smale) sequence at level $c$ is a sequence $\left(x_{n}\right)_{n}$ such that $\left\|I^{\prime}\left(x_{n}\right)\right\| \rightarrow 0$ and $I\left(x_{n}\right) \rightarrow c$.

Given a family $\mathcal{F}$ of subsets of $X$, and a function $I$ over $X$, the inf-max of $I$ over $\mathcal{F}$ is the (possibly infinite) number:

$$
c(I, \mathcal{F})=\inf _{A \in \mathcal{F}} \max _{x \in A} I(x) .
$$

Under certain circumstances, $c(I, \mathcal{F})$ will be a critical value. We first describe a fairly general setting where this is the case.

Let $\left\{T_{g} ; g \in G\right\}$ be a representation of a topological group $G$ over a manifold $X$. Say that a function $I: X \rightarrow \mathbf{R}$ is $G$-invariant if $I\left(T_{g} x\right)=I(x)$ for all $x \in X$ and $g \in G$. A subset $A$ of $X$ is $G$-invariant if its characteristic function is. A map $F: X \rightarrow X$ is said to be $G$-equivariant if $F\left(T_{g} x\right)=T_{g} F(x)$ for $x \in X$ and $g \in G$. A homotopy $\eta:[0,1] \times X \rightarrow X$ will be called $G$-equivariant if for each $t \in[0,1]$, the map $\eta(t, \cdot)$ is equivariant from $X$ into itself. Denote by $C(D, X)$ the class of continuous maps from $D$ into $X$ and by $C_{G}(D, X)$ the subset of those that are $G$-equivariant.

Definition 2.1. Let $B$ be a closed subset of $X$. Say that a class $\mathcal{F}$ of compact subsets of $X$ is a $G$-homotopy stable family with boundary $B$ provided:

(a) every set in $\mathcal{F}$ is $G$-invariant and contains $B$;

(b) for any set $A$ in $\mathcal{F}$ and any $G$-equivariant deformation $\eta$ of the identity that leaves $B$ invariant 1$]$ we have $\eta(\{1\} \times A) \in \mathcal{F}$.

In the absence of a group action (i.e., when $G$ consists only of the identity), we simply say that $\mathcal{F}$ is homotopy stable with boundary $B$. The most elementary example (besides the class of all subsets which corresponds to minimization) is the mountain pass family, since $\mathcal{F}_{u_{0}}^{u_{1}}$ is clearly homotopy stable with boundary $B=\left\{u_{0}, u_{1}\right\}$. Many other examples of homotopy stable families will appear below. The following min-max principle for locating critical levels essentially goes back to G. Birkhoff.

Proposition 2.2. Let $G$ be a discrete (or a compact Lie) group acting (differentiably) on a complete connected $C^{1}$-Finsler manifold $X$ without boundary. Let I be a $G$-invariant $C^{1}$-functional on $X$ and consider a $G$-homotopy stable family $\mathcal{F}$ with a closed boundary B. Assume

$$
\sup I(B)<c:=c(I, \mathcal{F}) .
$$

Then, for any min-maxing sequence $\left(A_{n}\right)_{n}$ in $\mathcal{F}$ (i.e., $\max _{A_{n}} I \rightarrow c$ ), there exists a sequence $\left(x_{n}\right)_{n}$ in $X$ such that $\lim _{n} I\left(x_{n}\right)=c, \lim _{n}\left\|I^{\prime}\left(x_{n}\right)\right\|=0$ and $\lim _{n} \operatorname{dist}\left(x_{n}, A_{n}\right)=0$.

\footnotetext{
${ }^{1} \eta \in C_{G}([0,1] \times X ; X) ; \eta(t, x)=x$ for $(t, x) \in(\{0\} \times X) \cup([0,1] \times B)$.
} 
Remark 2.2. (i) The above definition and proposition are still valid if the boundary $B$ is empty, provided we follow the usual convention of defining $\sup (\emptyset)=-\infty$. In this case, we will simply say that $\mathcal{F}$ is a $G$-homotopy stable family.

(ii) One needs the family $\mathcal{F}$ to be stable only under those homotopies along which the functional $I$ is decreasing. Actually, stability under the flow generated by the negative gradient field of $I$ is sufficient.

(iii) One can sometimes do without the compactness of the sets in $\mathcal{F}$, and the deformations in Definition 2.1 may be replaced by a less general class. For example, if the gradient of $I$ is of the form $U+K$ with $U$ an invertible linear operator and $K$ compact, then the setup can be much more infinite dimensional as long as the class $\mathcal{F}$ is suitably defined. We shall see an example in Section 10. (See also Benci-Rabinowitz [B-R].)

The above proposition identifies a potential critical level. The problem of existence of a critical point then reduces to proving that a sequence $\left(x_{n}\right)_{n}$ satisfying $\lim _{n} I\left(x_{n}\right)=c$ and $\lim _{n}\left\|I^{\prime}\left(x_{n}\right)\right\|=0$ is relatively compact in $X$. This is usually where the hard analysis is needed. Any function possessing such a property is said to satisfy the Palais-Smale condition at level $c$ : in short $(\mathrm{PS})_{c}$. It is of course sufficient to have compactness of those approximate critical points $\left(x_{n}\right)_{n}$ that are arbitrarily close to one particular min-maxing sequence $\left(A_{n}\right)_{n}$ in $\mathcal{F}$. See Example (3) below.

Interestingly, various relevant functionals originating in physics and in differential geometry do not satisfy this (PS) condition, or they may only satisfy it for certain energy levels (Example 2) or under additional second order information (Example 15) or both (Example 4) or along gradient lines like in the work of Bahri [Ba] and others Ba-Co1. These problems usually occur in situations involving the critical exponent in the Sobolev embedding theorem, or in cases where scale or gauge invariance requirements give rise to non-compact group actions. Many of the variational methods discussed below are geared towards dealing with such situations.

Example (1): An elliptic problem with a Hardy-type singularity. Problem (2.1) in its full generality has been studied in [G-Y]. We concentrate here and in section 3 on two simple cases that are interesting enough to illustrate the usefulness of the methods. We deal with the regular Laplacian $(p=2)$ and a superlinear perturbation $(r>2)$ of a Hardy-type singularity $q=s=2$.

Proposition 2.3. Consider the following equation in dimension $n \geq 3$ :

$$
\left\{\begin{aligned}
-\Delta u & =\lambda|u|^{r-2} u+\mu \frac{u}{|x|^{2}} & & \text { in } \Omega \subset \mathbf{R}^{n} \\
u & =0 & & \text { on } \partial \Omega .
\end{aligned}\right.
$$

(i) If $2<r<2^{*}$ and $0<\mu<\mu_{2}(\Omega)$ (the best constant in Hardy's inequality), then (2.10) has a positive solution for any $\lambda>0$.

(ii) If $r=2^{*}$ (Sobolev-critical non-singular term) and $\Omega$ is star-shaped, then (2.10) has no non-trivial solution for any $\lambda>0, \mu>0$.

The second statement has been established above. For the existence result, one easily notices a mountain-pass configuration. Actually, 0 is a local minimum for 
$I_{\lambda, \mu}$ since:

$$
I_{\lambda, \mu}(u) \geq\left(1-\frac{\mu}{\mu_{2}}\right) \frac{1}{2} \int_{\Omega}|\nabla u|^{2} d x-\frac{\lambda}{r} \int_{\Omega}|u|^{r} d x \geq C_{1}\|u\|_{H_{0}^{1}}^{2}-C_{2}\|u\|_{H_{0}^{1}}^{r} .
$$

On the other hand, $\inf _{H_{0}^{1}} I_{\lambda, \mu}=-\infty$, since for $u \in H_{0}^{1}(\Omega)$ and $R>0$,

$$
I_{\lambda, \mu}(R u) \leq \frac{R^{2}}{2}\|u\|_{H_{0}^{1}(\Omega)}^{2}-\frac{R^{r}}{r}\|u\|_{r}^{r} .
$$

So, it remains to show that since $r<2^{*}$ and $0<\mu<\mu_{2}$, the functional

$$
I_{\lambda, \mu}(u)=\frac{1}{2} \int_{\Omega}|\nabla u|^{2} d x-\frac{\mu}{2} \int_{\Omega} \frac{|u|^{2}}{|x|^{2}}-\frac{\lambda}{r} \int_{\Omega}|u|^{r} d x
$$

satisfies the Palais-Smale condition at any energy level. Indeed, if $\left\{u_{n}\right\}$ in $H_{0}^{1}(\Omega)$ satisfies $I_{\lambda, \mu}\left(u_{n}\right) \rightarrow c$ and $I_{\lambda, \mu}^{\prime}\left(u_{n}\right) \rightarrow 0$, then for any $v \in C_{0}^{\infty}(\Omega)$,

$$
\left\langle I_{\lambda, \mu}^{\prime}\left(u_{n}\right), v\right\rangle=\int_{\Omega}\left(\left\langle\nabla u_{n}, \nabla v\right\rangle-\lambda\left|u_{n}\right|^{r-2} u_{n} v-\mu \frac{u_{n}}{|x|^{2}} v\right) d x
$$

so that:

$$
o(1)\left(1+\left\|u_{n}\right\|_{H_{0}^{1}}\right)+2 c \geq 2 I_{\lambda, \mu}\left(u_{n}\right)-\left\langle u_{n}, I_{\lambda, \mu}^{\prime}\left(u_{n}\right)\right\rangle=\lambda\left(1-\frac{2}{r}\right)\left\|u_{n}\right\|_{r}^{r} .
$$

Combining the latter equation with (2.11), we get $\left\|u_{n}\right\|_{H_{0}^{1}}^{2} \leq C+o(1)\left\|u_{n}\right\|_{H_{0}^{1}}$. Hence $\left(u_{n}\right)$ is bounded, and we may assume that $u_{n} \rightarrow u$ weakly in $H_{0}^{1}(\Omega)$. From (2.12) we get for any $v \in C_{0}^{\infty}(\Omega)$,

$$
0=\int_{\Omega}\left(\langle\nabla u, \nabla v\rangle-\lambda|u|^{r-2} u v-\mu \frac{u}{|x|^{2}} v\right) d x=\left\langle I_{\lambda, \mu}^{\prime}(u), v\right\rangle
$$

Hence $u \in H_{0}^{1}(\Omega)$ is a weak solution of (2.10). Since $\left\|u_{n}-u\right\|_{r} \rightarrow 0$, we have $I_{\lambda, \mu}\left(u_{n}\right)=I_{\lambda, \mu}(u)+I_{0, \mu}\left(u_{n}-u\right)+o(1)$; and combining this with (2.13) after choosing $v=u$, we get

$$
\begin{array}{r}
o(1)=\left\langle I_{\lambda, \mu}^{\prime}\left(u_{n}\right), u_{n}-u\right\rangle=\left\langle I_{\lambda, \mu}^{\prime}\left(u_{n}\right)-I_{\lambda, \mu}^{\prime}(u), u_{n}-u\right\rangle \\
=\int_{\Omega}\left(\left|\nabla u_{n}-\nabla u\right|^{2}-\mu \frac{\left|u_{n}-u\right|^{2}}{|x|^{2}}\right)+o(1) .
\end{array}
$$

We obtain strong convergence since $\mu<\mu_{2}$, because then

$o(1)=\int_{\Omega}\left(\left|\nabla u_{n}-\nabla u\right|^{2}-\mu \frac{\left|u_{n}-u\right|^{2}}{|x|^{2}}\right)+o(1) \leq\left(1-\frac{\mu}{\mu_{2}}\right) \int_{\Omega}\left|\nabla u_{n}-\nabla u\right|^{2}+o(1)$.

2.3. Analysis of Palais-Smale sequences in borderline variational problems. One of the most influential ideas in the modern era of variational calculus is probably the new belief that the failure of the Palais-Smale condition is not always the final word and that a finer analysis of the behavior of non-convergent (PS) sequences may require new variational methods that would prevent such an eventuality. The first to make such an analysis was T. Aubin [Au], who noted that in the context of the Yamabe problem, the Palais-Smale condition can hold up to certain energy levels related to the best constant in the Sobolev inequality. Another important technique for analyzing non-compactness appeared in the celebrated work of Sacks-Uhlenbeck [S-U] where they introduce, in their study of harmonic maps, the method of blowing up the singularities near points of concentration. Since then, this phenomenon has been detected in prescribed curvature problems of Rellich type [B-C], Yang-Mills equations [Tau1], closed geodesics problems [Tan2] and 
many other situations. The main idea is that often a non-convergent Palais-Smale sequence - or a blown up version of it - splits up into a piece that converges weakly to a solution of the original problem and another one that converges to solutions of a closely related "limiting problem". The appearance of the second part - which usually prevents compactness - can sometimes be avoided through energy restrictions and/or spectral confinement. For example, blowing-up non-convergent Palais-Smale sequences of problems of the type:

$$
\left\{\begin{aligned}
-\Delta u & =f(u) & & \text { in } \Omega \\
u & =0 & & \text { on } \partial \Omega
\end{aligned}\right.
$$

where the non-linearity satisfies $\lim _{|u| \rightarrow \infty} \frac{f(u)}{|u|^{p}}=1$, leads to entire solutions of the "limiting" equation $-\Delta u=u^{p}$ on $\mathbf{R}^{n}$. Proving compactness then reduces to establishing Liouville-type results of the following type: If $p<\frac{2 n}{n-2}$ (subcritical case), then all entire solutions in $H^{1}\left(\mathbf{R}^{n}\right)$ of the limiting equation are trivial. This again follows from the Pohozaev identity.

We now describe two examples where a more careful analysis of Palais-Smale sequences is needed. One was chosen for its analytic relevance and another for its geometric appeal.

Example (2): Elliptic problems with a critical Hardy-Sobolev exponent. We deal with a linear perturbation $(r=2)$ of a Hardy-Sobolev critical non-linearity, i.e., $q=2^{*}(s)=\frac{2(n-s)}{n-2}$ and $0 \leq s<2<n$. The equation is then

$$
\left\{\begin{aligned}
-\Delta u & =\lambda u+\frac{|u|^{q-2}}{|x|^{s}} u & & \text { in } \Omega \\
u & =0 & & \text { on } \partial \Omega,
\end{aligned}\right.
$$

where $\lambda>0$ and $\Omega$ is a domain in $\mathbf{R}^{n}$. The corresponding energy functional

$$
I_{\lambda}(u)=\frac{1}{2} \int_{\Omega}|\nabla u|^{2} d x-\frac{\lambda}{2} \int_{\Omega}|u|^{2} d x-\frac{1}{2^{*}(s)} \int_{\Omega} \frac{|u|^{2^{*}(s)}}{|x|^{s}} d x
$$

is then well defined on the Sobolev space $H_{0}^{1}(\Omega)$.

If 0 belongs to the interior of $\Omega$, then - as in the case when $s=0$ - the best constant $\mu_{s}(\Omega)$ in the Hardy-Sobolev inequality is independent of the domain $\Omega$ and is attained when $\Omega=\mathbf{R}^{n}$ by the functions

$$
U_{a}(x)=(a(n-s)(n-2))^{\frac{n-2}{2(2-s)}}\left(a+|x|^{2-s}\right)^{\frac{2-n}{2-s}}
$$

for some $a>0$. Moreover the functions $U_{a}$ are the only positive radial solutions of the "limiting problem"

$$
\left\{\begin{aligned}
-\Delta u & =\frac{|u|^{2^{*}(s)-2} u}{|x|^{s}} & & \text { on } \mathbf{R}^{n} \\
u(x) & \rightarrow 0 \text { when } & & \|x\| \rightarrow \infty
\end{aligned}\right.
$$

whose solutions are the critical points of the functional $I_{0}$ on $D^{1,2}\left(\mathbf{R}^{n}\right)[\mathrm{G}-\mathrm{Y}]$.

Hence,

$$
\mu_{s}\left(\mathbf{R}^{n}\right)\left(\int_{\mathbf{R}^{n}} \frac{\left|U_{a}\right|^{q}}{|x|^{s}}\right)^{\frac{2}{q}}=\left\|\nabla U_{a}\right\|_{2}^{2}=\int_{\mathbf{R}^{n}} \frac{\left|U_{a}\right|^{q}}{|x|^{s}}=\mu_{s}\left(\mathbf{R}^{n}\right)^{\frac{n-s}{2-s}} .
$$

If 0 belongs to the boundary of $\Omega$, the situation is more interesting as Egnell $\mathrm{Eg}$. has shown that $\mu_{s}(\mathcal{C})$ is attained for any proper infinite cone $\mathcal{C}$ with vertex 0 and therefore $\mu_{s}\left(\mathbf{R}^{n}\right)<\mu_{s}(\mathcal{C})$. On the other hand, one can show $\mathrm{G}-\mathrm{K}$ that if the boundary of $\Omega$ is smooth at 0 , then $\mu_{s}(\Omega) \leq \mu_{s}\left(\mathbf{R}_{+}^{n}\right)$. 
The following proposition describing how to blow up singularities is by now standard. It can be found - in the case $s=0$ - in the book of Struwe ( $\mathrm{St}$, p. 169).

Proposition 2.4. Suppose $\left(u_{m}\right)_{m}$ is a bounded sequence in $H_{0}^{1}(\Omega)$ that satisfies $I_{\lambda}\left(u_{m}\right) \rightarrow c$ and $I_{\lambda}^{\prime}\left(u_{m}\right) \rightarrow 0$ strongly in $H^{-1}(\Omega)$ as $m \rightarrow \infty$. Then, there is an integer $k \geq 0$, a solution $U^{0}$ of $(2.14)$ in $H_{0}^{1}(\Omega)$, solutions $U^{1}, \ldots, U^{k}$ of $(2.16)$ in $D^{1,2}\left(\mathbf{R}^{n}\right)$, sequences of radii $r_{m}^{1}, \ldots, r_{m}^{k}>0$ such that for some subsequence $m \rightarrow \infty, r_{m}^{k} \rightarrow 0$ and:

(i) $u_{m} \rightarrow U^{0}$ weakly in $H_{0}^{1}(\Omega)$;

(ii) $\left\|u_{m}-U^{0}-\Sigma_{j=1}^{k}\left(r_{m}^{j}\right)^{\frac{2-n}{2}} U^{j}\left(r_{m}^{j} \bullet\right)\right\| \rightarrow 0$;

(iii) $\left\|u_{m}\right\|_{1}^{2} \rightarrow \sum_{j=0}^{k}\left\|U^{j}\right\|_{1}^{2}$ where $\|u\|_{1}$ is the norm in $L^{1}\left(|x|^{-s} d s\right)$;

(iv) $I_{\lambda}\left(u_{m}\right) \rightarrow I_{\lambda}\left(U^{0}\right)+\sum_{j=1}^{k} I_{0}\left(U^{j}\right)$.

In the case where $s=0, I_{\lambda}$ and $I_{0}$ are both invariant under translation and dilations and therefore one can be more precise in capturing the "blow up" near the points of concentration. In addition to the above, one then obtains sequences of points $x_{m}^{1}, \ldots, x_{m}^{k} \in \mathbf{R}^{n}$ such that $u_{m}^{0} \equiv u_{m}$ and $u_{m}^{j} \equiv\left(u_{m}^{j-1}-U^{j-1}\right)_{r_{m}^{j}, x_{m}^{j}} \rightarrow U^{j}$ in $H^{1}$ for $j=1, \ldots, k$, where for any function $v$, we have denoted by $v_{r, x_{0}}$ the function $r^{\frac{2-n}{2}} u\left(r\left(\bullet-x_{0}\right)\right)$.

We are now interested in the existence of a first solution for (2.14). We shall come back to this equation in the next section in order to deal with the question of multiplicity and compactness at higher energy levels. Define a mountain pass class

$$
\mathcal{F}_{1}=\left\{h \in C^{1}\left([0,1] ; H_{0}^{1}(\Omega)\right) ; h(0)=0, I_{\lambda}(h(1))<0\right\} .
$$

Proposition 2.5. Suppose $\Omega$ is a bounded smooth domain containing 0 in its interior. If $n \geq 4$ and $0<\lambda<\lambda_{1}(\Omega)$, then the following hold:

(i) $I_{\lambda}$ satisfies $(P S)_{c}$ for any $c<\frac{2-s}{2(n-s)} \mu_{s}\left(\mathbf{R}^{n}\right)^{\frac{n-s}{2-s}}$.

(ii) $0<c_{1}:=\inf _{h \in \mathcal{F}_{1}} \sup _{t \in[0,1]} I_{\lambda}(h(t))<\frac{2-s}{2(n-s)} \mu_{s}\left(\mathbf{R}^{n}\right)^{\frac{n-s}{2-s}}$ and problem (2.14) has a solution $u_{1}$ at energy level $c_{1}$ that is strictly positive on $\Omega$.

Indeed, the Palais-Smale condition is due to the fact that below that energy level, estimate (2.17) and Proposition 2.4.(iv) leave no room for any "bubble" $U^{j}, j \geq 1$ (since $I_{0}\left(U^{j}\right) \geq \frac{2-s}{2(n-s)} \mu_{s}^{\frac{n-s}{2-s}}$ ) and therefore convergence holds in view of Proposition 2.4.(iii).

In order to estimate the energy level $c_{1}$, consider the functions $U_{\varepsilon}(x)=$ $k(\epsilon)\left(\varepsilon+|x|^{2-s}\right)^{\frac{2-n}{2-s}}$ where the best constant in the Sobolev-Hardy inequality is attained. Let $0 \leq \varphi(x) \leq 1$ be a cut-off function in $C_{0}^{\infty}(\Omega)$ defined as $\varphi(x)=1$ if $|x| \leq R$ and 0 if $|x| \geq 2 R$ where $B_{2 R}(0)$ is a ball in $\Omega$. Set $v_{\varepsilon}(x)=\varphi(x) U_{\varepsilon}(x)$, and consider the function

$$
g(t)=I_{\lambda}\left(t v_{\varepsilon}\right)=\frac{t^{2}}{2} \int_{\Omega}\left|\nabla v_{\varepsilon}\right|^{2}-\frac{t^{2^{*}(s)}}{2^{*}(s)} \int_{\Omega} \frac{\left|v_{\varepsilon}\right|^{2^{*}(s)}}{|x|^{s}} d x-\frac{\lambda t^{2}}{2} \int_{\Omega}\left|v_{\varepsilon}\right|^{2} .
$$

Since $\lambda<\lambda_{1}$, we have $g(t)>0$ when $t$ is close to 0 and $\lim _{t \rightarrow \infty} g(t)=-\infty$. So $g(t)$ attains its maximum at the point $t_{\varepsilon}=\left(\int_{\Omega}\left|\nabla \bar{v}_{\varepsilon}\right|^{2}-\lambda \int_{\Omega}\left|\bar{v}_{\varepsilon}\right|^{2}\right)^{\frac{1}{2^{*}(s)-2}}$ at level

$$
g\left(t_{\varepsilon}\right)=\left(\frac{1}{2}-\frac{1}{2^{*}(s)}\right)\left(\int_{\Omega}\left|\nabla \bar{v}_{\varepsilon}\right|^{2}-\lambda \int_{\Omega}\left|\bar{v}_{\varepsilon}\right|^{2}\right)^{\frac{2^{*}(s)}{2^{*}(s)-2}},
$$


where $\bar{v}_{\epsilon}(x):=\frac{v_{\epsilon}(x)}{\int_{\Omega} \frac{\left|v_{\varepsilon}\right|^{2 *}(s)}{|x|^{s}} d x}$. For $\varepsilon \rightarrow 0$, the behavior of $v_{\varepsilon}$ is essentially the same as $U_{\varepsilon}$, but precise estimates for the error terms are needed. We refer to [G-Y] for the following estimates (to $[\mathrm{B}-\mathrm{N}]$ for the case $s=0$ ):

$$
g\left(t_{\varepsilon}\right)= \begin{cases}\frac{2-s}{2(n-s)} \mu_{s}^{\frac{n-s}{2-s}}+O\left(\varepsilon^{\frac{n-2}{2-s}}\right)-O\left(\varepsilon^{\frac{2}{2-s}}\right) & n>4 \\ \frac{2-s}{2(n-s)} \mu_{s}^{\frac{n-s}{2-s}}+O\left(\varepsilon^{\frac{n-2}{2-s}}\right)-O\left(\varepsilon^{\frac{n-2}{2-s}}|\log \varepsilon|\right) & n=4 .\end{cases}
$$

Remark 2.3. In the same spirit one can establish the following G-K]: Suppose $\Omega$ is a bounded smooth domain in $\mathbf{R}^{n}$ containing 0 on its boundary.

(i) If $n \geq 4,0<\lambda<\lambda_{1}(\Omega)$ and if the boundary of $\Omega$ is flat at 0 , then equation (2.14) with Dirichlet boundary conditions has a positive solution on $\Omega$.

(ii) If $n \geq 3, \lambda<0$ and if the boundary has positive mean curvature at 0 , then equation (2.14) with Neuman boundary conditions has a positive solution on $\Omega$.

Example (3): Closed geodesics on $\left(\mathbf{R} \times S^{N-1}, g\right)$ and periodic solutions for singular Hamiltonian systems. We chose this problem - recently studied by Tanaka Tan2 - first for its geometric appeal $\left(\mathbf{R} \times S^{N-1}\right.$ being the simplest nontrivial non-compact Riemannian manifold) but also as a test case for many variations of the Palais-Smale condition, even though the lack of compactness is not severe since it is due to the non-boundedness of the solutions' target space. The setting is also relevant to the problem of existence of periodic solutions with prescribed energy for certain singular Hamiltonian systems (Corollary 2.1). This topic, pioneered by Poincaré, is currently witnessing progress by way of modern variational methods (see $[\mathrm{A}-\mathrm{CZ}$ and its references).

Closed geodesics on $\left(\mathbf{R} \times S^{N-1}, g\right)$ where $g$ is a complete Riemannian metric can be characterized as critical points of the functional $I: \Lambda \rightarrow \mathbf{R}$ defined by

$$
I(u)=\frac{1}{2} \int_{0}^{1} g_{u}(\dot{u}, \dot{u}) d t
$$

on $\Lambda:=\left\{u \in H^{1}\left(0,1 ; \mathbf{R} \times S^{N-1}\right) ; u(0)=u(1)\right\}$, the loop space of 1 -periodic curves on $\mathbf{R} \times S^{N-1}$. $\Lambda$ is a $C^{\infty}$ Hilbert manifold with tangent space at a point $u(t)=$ $(s(t), x(t)) \in \Lambda$ given by

$$
\begin{array}{r}
T_{u} \Lambda=\left\{(\xi, \eta) \in H^{1}\left([0,1] ; \mathbf{R} \times \mathbf{R}^{N}\right) ;(\xi, \eta)(0)=(\xi, \eta)(1)\right. \\
\text { and } \left.(\xi(t), \eta(t)) \in \mathbf{R} \times T_{u(t)} S^{N-1}\right\} .
\end{array}
$$

Equip $\Lambda$ with the Riemannian structure

$$
\left\langle\left(\xi_{1}, \eta_{1}\right),\left(\xi_{2}, \eta_{2}\right)\right\rangle_{T_{(s, x)} \Lambda}=\int_{0}^{1}\left[\dot{\xi}_{1} \dot{\xi}_{2}+D_{t} \eta_{1} \cdot D_{t} \eta_{2}\right] d t+\xi_{1}(0) \xi_{2}(0)+\eta_{1}(0) \cdot \eta_{2}(0)
$$

for $\left(\xi_{1}, \eta_{1}\right),\left(\xi_{2}, \eta_{2}\right) \in T_{(s, x)} \Lambda$ and $(s, x) \in \Lambda$. Here $D_{t} \eta$ is the covariant derivative of $\eta(t)$. Write $\operatorname{dist}_{\Lambda}(\cdot, \cdot)$ for the distance on $\Lambda$ induced by the Riemannian structure $\langle\cdot, \cdot\rangle_{T \Lambda}$. On $\Lambda$, the metric dist $_{\Lambda}$ is equivalent to the norm on $H^{1}\left([0,1] ; \mathbf{R} \times \mathbf{R}^{N}\right)$ and $\left(\Lambda, \operatorname{dist}_{\Lambda}(\cdot, \cdot)\right)$ is a complete metric space.

The following proposition describes the breakdown of the Palais-Smale condition for $I$ under the condition that $g$ is a Riemannian metric on $\mathbf{R} \times S^{N-1}$ satisfying $g \sim$ $g^{0}$ as $s \sim \pm \infty$, where $g^{0}$ is the standard metric. More precisely, if $\left(\xi^{1}, \cdots, \xi^{N-1}\right)$ 
are local coordinates of $S^{N-1}$ in an open set $U \subset S^{N-1}$ and setting $\xi^{0}=s$, we write

$g=\sum_{i, j=0}^{N-1} g_{i j}\left(\xi^{0}, \xi^{1}, \cdots, \xi^{N-1}\right) d \xi^{i} \otimes d \xi^{j}$ and $g^{0}=\sum_{i, j=0}^{N-1} g_{i j}^{0}\left(\xi^{0}, \xi^{1}, \cdot, \xi^{N-1}\right) d \xi^{i} \otimes d \xi^{j}$.

Note that $g_{i j}^{0}$ is independent of $\xi^{0}=s$ and the hypothesis $g \sim g^{0}$ as $s \sim \pm \infty$ means that:

$$
\begin{cases}g_{i j}\left(s, \xi^{1}, \cdots, \xi^{N-1}\right) \rightarrow g_{i j}^{0}\left(\xi^{1}, \cdots, \xi^{N-1}\right) & \text { in } C^{2}(U, \mathbf{R}) \quad \text { and } \\ \frac{\partial g_{i j}}{\partial s}\left(s, \xi^{1}, \cdots, \xi^{N-1}\right) \rightarrow 0 \quad \text { in } C^{1}(U, \mathbf{R}) & \text { as }|s| \rightarrow \infty .\end{cases}
$$

Proposition 2.6. Let $I$ be the functional associated to a Riemannian metric $g$ on $\mathbf{R} \times S^{N-1}$ that satisfies $g \sim g^{0}$ as $s \sim \pm \infty$. Suppose that $\left(u_{j}\right)_{j=1}^{\infty} \subset \Lambda$ satisfies $\left\|I^{\prime}\left(u_{j}\right)\right\|_{\left(T_{u_{j}} \Lambda\right)^{*}} \rightarrow 0$ and $I\left(u_{j}\right) \rightarrow c$ for some $c>0$. Then, there is a subsequence - still denoted by $u_{j}$ - such that one of the following 2 statements holds:

(i) There is a non-constant closed geodesic $u_{0} \in \Lambda$ on $\left(\mathbf{R} \times S^{N-1}, g\right)$ such that $u_{j} \rightarrow u_{0}$ in $\Lambda$ or equivalently $\left\|u_{j}-u_{0}\right\|_{H^{1}\left(0,1 ; \mathbf{R} \times \mathbf{R}^{N}\right)} \rightarrow 0$.

(ii) There is a closed geodesic $x_{0}(t) \in \Lambda_{S^{N-1}}$ on the standard sphere $S^{N-1}$ such that if we write $u_{j}(t)=\left(s_{j}(t), x_{j}(t)\right)$, then

(1) $s_{j}(0) \rightarrow \infty$ or $s_{j}(0) \rightarrow-\infty$.

(2) $\tilde{u}_{j}(t) \equiv\left(s_{j}(t)-s_{j}(0), x_{j}(t)\right) \rightarrow\left(0, x_{0}(t)\right)$ in $\Lambda$ (equivalently, $\left\|\dot{s}_{j}\right\|_{L^{2}(0,1)} \rightarrow 0$ and $\left.\left\|x_{j}-x_{0}\right\|_{H^{1}\left(0,1 ; \mathbf{R}^{N}\right)} \rightarrow 0\right)$.

Case (ii) occurs if and only if $c=2 \pi^{2} k^{2}$ for some $k \in \mathbf{N}$; $x_{0}(t)$ is then a great circle of the form $y_{k}(t)=e_{1} \cos 2 \pi k t+e_{2} \sin 2 \pi k t$ where $k \in \mathbf{Z}$ and $e_{1}, e_{2} \in \mathbf{R}^{N}$ are orthonormal vectors.

Here again, we have a limiting problem at infinity. The great circles $y_{k}$ are the closed geodesics on the standard sphere $S^{N-1}=\left\{x \in \mathbf{R}^{N} ;|x|=1\right\}$ which can be characterized as the critical points of $I_{S^{N-1}}(x)=\frac{1}{2} \int_{0}^{1}|\dot{x}|^{2} d t$ on the space

$$
\Lambda_{S^{N-1}}=\left\{x(t) \in H^{1}\left([0,1] ; \mathbf{R}^{N}\right) ; x(0)=x(1),|x(t)|=1 \text { for all } t \in[0,1]\right\} .
$$

Their critical values are $I_{S^{N-1}}\left(y_{k}\right)=2 \pi^{2} k^{2}$ where $k \in \mathbf{Z}$, and the min-max characterisation of these closed geodesics goes as follows:

If $N=2$, set $\Sigma_{S^{1}}=\left\{u \in \Lambda_{S^{N-1}}\right.$; Winding number of $\left.u=1\right\}$. Then, $\inf _{u \in \Sigma_{S^{1}}} I_{S^{1}}(u)$ $=2 \pi^{2}$ which corresponds to the prime closed geodesic $y_{1}(t)$.

If $N \geq 3$, set $\Sigma_{S^{N-1}}=\left\{\sigma \in C\left(S^{N-2}, \Lambda_{S^{N-1}}\right) ; \operatorname{deg} \tilde{\sigma}=1\right\}$ where $\tilde{\sigma}: S^{N-2} \times$ $([0,1] /\{0,1\}) \simeq S^{N-2} \times S^{1} \rightarrow S^{N-1}$ is defined by $\tilde{\sigma}(z, t)=\sigma(z)(t)$ for $\sigma \in$ $C\left(S^{N-2}, \Lambda_{S^{N-1}}\right)$ and $\operatorname{deg} \tilde{\sigma}$ is its Brouwer degree. The min-max value

$$
c=\inf _{\sigma \in \Sigma_{S^{N-1}}} \max _{z \in S^{N-2}} I_{S^{N-1}}(\sigma(z))
$$

gives a non-zero critical value for $I_{S^{N-1}}$; hence $c=2 \pi^{2} k^{2}$ for some $k \in \mathbf{N}$. On the other hand, we find for a suitable $\sigma_{0}(z) \in \Sigma_{S^{N-1}}$,

$$
\max _{z \in S^{N-2}} I_{S^{N-1}}\left(\sigma_{0}(z)\right)=2 \pi^{2},
$$


so that $c=2 \pi^{2}$, corresponding to a prime closed geodesic $y_{1}(t)$. An example of $\sigma_{0}$ that will be used below is

$$
\begin{aligned}
& \sigma_{0}(z)(t)= \\
& \begin{cases}\left(2 z_{1}, \cdots, 2 z_{N-2}, \sqrt{4 z_{N-1}^{2}-3} \cos 2 \pi t, \sqrt{4 z_{N-1}^{2}-3} \sin 2 \pi t\right) & \text { if }\left|z_{N-1}\right| \geq \sqrt{3} / 2, \\
\left(\frac{2\left|z_{N-1}\right|}{\sqrt{3}} \frac{z_{1}}{\sqrt{1-z_{N-1}^{2}}}, \cdots, \frac{2\left|z_{N-1}\right|}{\sqrt{3}} \frac{z_{N-2}}{\sqrt{1-z_{N-1}^{2}}}, \sqrt{\frac{3-4 z_{N-1}^{2}}{3}}, 0\right) & \text { if }\left|z_{N-1}\right|<\sqrt{3} / 2,\end{cases}
\end{aligned}
$$

where $z=\left(z_{1}, \cdots, z_{N-1}\right) \in S^{N-2}=\left\{z \in \mathbf{R}^{N-1} ;|z|=1\right\}$.

We now start sketching the proof of the following proposition. It will be completed in Section 8, where improved variational principles will deal with cases where there is lack of compactness.

Proposition 2.7. Let $g$ be a Riemannian metric on $\mathbf{R} \times S^{N-1}$ and suppose that $g \sim g^{0}$ as $s \sim \pm \infty$. Then $\left(\mathbf{R} \times S^{N-1}, g\right)$ has at least one non-constant closed geodesic.

This example of a non-compact Riemannian manifold is of interest because of the one-to-one correspondence between the periodic solutions of certain singular Hamiltonian systems with potential $V$, say, and the non-constant closed geodesics on $\left(\mathbf{R} \times S^{N-1}, g^{V}\right)$ where $g^{V}$ is an appropriate metric on $\mathbf{R} \times S^{N-1}$ associated to the potential $V$. One obtains the following consequence. See Tanaka for details Tan2.

Corollary 2.1. There exists a periodic solution with a prescribed energy to the following Hamiltonian system:

$$
\left\{\begin{array}{l}
\ddot{q}+\nabla V(q)=0 \\
\frac{1}{2}|\dot{q}|^{2}+V(q)=0
\end{array}\right.
$$

provided $V(q)<0$ for all $q \in \mathbf{R}^{N} \backslash\{0\}$ and $V(q) \sim-\frac{1}{|q|^{2}}$ as $|q| \sim 0$ and $|q| \sim \infty$.

To establish Proposition 2.7, one defines two min-max values that have the potential to be critical levels for $I$. We deal with the case $N \geq 3$ as the 2-dimensional case can be handled by similar methods. For an $(N-2)$-dimensional compact manifold $M$, set

$$
\Gamma(M)=\{\gamma \in C(M, \Lambda) ; \gamma(M) \text { is not contractible in } \Lambda\},
$$

and consider the following class of $(N-2)$-dimensional compact manifolds:

$$
\mathcal{M}_{N-2}=\{M ; M \text { connected and } \Gamma(M) \neq \emptyset\} .
$$

Note that $S^{N-2} \in \mathcal{M}_{N-2}$ because of the existence of $\sigma_{0}(z)$ given in (2.22) so that $\mathcal{M}_{N-2} \neq \emptyset$. For $M \in \mathcal{M}_{N-2}$, set

$$
b(M)=\inf _{\gamma \in \Gamma(M)} \max _{u \in \gamma(M)} I(u)
$$

and

$$
\underline{b}=\inf _{M \in \mathcal{M}_{N-2}} b(M) .
$$


To define the second min-max value, consider the following class of mappings:

$$
\bar{\Gamma}=\left\{\gamma \in C\left(\mathbf{R} \times S^{N-2}, \Lambda\right) ; \gamma(r, z)(t)=\left(r, \sigma_{0}(z)(t)\right) \text { for sufficiently large }|r|\right\} .
$$

It is clear that $\gamma_{0}(r, z)(t)=\left(r, \sigma_{0}(z)(t)\right) \in \bar{\Gamma}$; hence $\bar{\Gamma} \neq \emptyset$. Define

$$
\bar{b}=\inf _{\gamma \in \bar{\Gamma}} \sup _{u \in \gamma\left(\mathbf{R} \times S^{N-2}\right)} I(u) .
$$

Proposition 2.8. With the above notation, we have:

(i) $0<\underline{b} \leq 2 \pi^{2} \leq \bar{b}$.

(ii) Either $\underline{b}$ or $\bar{b}$ corresponds to a critical value of $I$.

To show that $0<\underline{b}$, first note that for $u=(s, x) \in \Lambda$,

$$
I(u)=\frac{1}{2} \int_{0}^{1} g_{u}(\dot{u}, \dot{u}) d t \geq \frac{m}{2} \int_{0}^{1}\left(|\dot{s}|^{2}+|\dot{x}|^{2}\right) d t \geq \frac{m}{2} \int_{0}^{1}|\dot{x}|^{2} d t=m I_{S^{N-1}}(x),
$$

for some constant $m>0$. Moreover, for any compact $(N-2)$-dimensional manifold $M$ such that

$$
\Gamma_{S^{N-1}}(M)=\left\{\sigma \in C\left(M, \Lambda_{S^{N-1}}\right) ; \sigma(M) \text { is not contractible in } \Lambda_{S^{N-1}}\right\} \neq \emptyset,
$$

we have $c\left(I_{S^{N-1}}, \Gamma_{S^{N-1}}(M)\right) \geq 2 \pi^{2}$, which yields that

$$
\underline{b}=\inf _{M \in \mathcal{M}_{N-2}} c(I, \Gamma(M)) \geq 2 \pi^{2} m>0 .
$$

To show that $\underline{b} \leq 2 \pi^{2}$, use that $S^{N-2} \in \mathcal{M}_{N-2}$ and set $\gamma_{\ell}(z)(t)=\left(\ell, \sigma_{0}(z)(t)\right)$ for $\ell \in \mathbf{R}$ and $z \in S^{N-2}$. Then $\gamma_{\ell}\left(S^{N-2}\right)$ is not contractible in $\Lambda$ and

$$
b\left(S^{N-2}\right) \leq \max _{z \in S^{N-2}} I\left(\gamma_{\ell}(z)\right)=\max _{z \in S^{N-2}} I\left(\ell, \sigma_{0}(z)\right) .
$$

Letting $\ell \rightarrow \infty$, we get from the condition on $g$ and estimate (2.21) that

$$
\underline{b} \leq b\left(S^{N-2}\right) \leq 2 \pi^{2} .
$$

To prove that $2 \pi^{2} \leq \bar{b}$, remark that for any $\gamma(r, z) \in \bar{\Gamma}$,

$$
\sup _{(r, z) \in \mathbf{R} \times S^{N-2}} I(\gamma(r, z)) \geq \limsup _{r \rightarrow \pm \infty} \max _{z \in S^{N-2}} I\left(r, \sigma_{0}(z)\right)=2 \pi^{2} .
$$

Now to prove (ii), we distinguish between the following 4 cases:

(A) $0<\underline{b}<2 \pi^{2}$,

(B) $\bar{b} \notin\left\{2 \pi^{2} k^{2} ; k \in \mathbf{N}\right\}$,

(C) $\bar{b} \in\left\{2 \pi^{2} k^{2} ; k \in \mathbf{N} \backslash\{1\}\right\}$,

(D) $\underline{b}=\bar{b}=2 \pi^{2}$.

Case (A) (or (B)) is immediate since, by Proposition 2.6, the functional $I$ satisfies the (PS) condition at such levels and $\underline{b}$ (or $\bar{b}$ ) is then a critical value of $I$.

Case (C) will have to wait till Section 8, where we show that $I$ satisfies a weaker form of the Palais-Smale condition which will eventually suffice.

For Case (D), we construct a particular min-maxing sequence $\left(A_{n}\right)_{n}$ in $\mathcal{F}=$ $\cup\left\{\Gamma(M) ; M \in \mathcal{M}_{N-2}\right\}$ verifying for each $n \in \mathbf{N}$ :

$$
s(0) \in[0,1] \text { for all } u(t)=(s(t), x(t)) \in A_{n} .
$$

By Proposition 2.2, one can then find an approximate critical sequence $\left(s_{n}, x_{n}\right)_{n}$ such that $\operatorname{dist}\left(\left(s_{n}, x_{n}\right), A_{n}\right) \rightarrow 0$. Such a sequence is then relatively compact because $\left(s_{n}\right)_{n}$ is bounded (Proposition 2.6). 
To construct $\left(A_{n}\right)_{n}$, one starts by using that $\bar{b}=2 \pi^{2}$ to find for any $\epsilon>0$, a $\gamma \in \bar{\Gamma}$ such that

$$
\sup _{(r, z) \in \mathbf{R} \times S^{N-2}} I(\gamma(r, z)) \leq 2 \pi^{2}+\epsilon .
$$

We may assume that $\gamma \in C^{\infty}\left(\mathbf{R} \times S^{N-2}, \Lambda\right) \cap \bar{\Gamma}$ and therefore write $\gamma(r, z)(t)=$ $(s(r, z)(t), x(r, z)(t))$. Consider $f(r, z)=s(r, z)(0)$ which is a $C^{\infty}$-mapping from $\mathbf{R} \times S^{N-2}$ to $\mathbf{R}$. Use Sard's theorem to find $\beta \in[0,1]$ such that $f(r, z)=\beta$ implies $\left(f_{r}(r, z), f_{z}(r, z)\right) \neq(0,0)$.

This means that $f^{-1}(\beta)$ is an $(N-2)$-dimensional submanifold of $\mathbf{R} \times S^{N-1}$. Since $f(r, z)=r$ for sufficiently large $|r|, f^{-1}(\beta)$ is compact and can be written as $f^{-1}(\beta)=M_{1} \cup M_{2} \cup \cdots \cup M_{n}$, where $M_{1}, M_{2}, \cdots, M_{n}$ are $(N-2)$-dimensional compact connected submanifolds of $\mathbf{R} \times S^{N-2}$. One then shows that there exists a $j_{0} \in\{1, \cdots, n\}$ such that $M_{j_{0}} \in \mathcal{M}_{N-2}$ and $\left.\gamma\right|_{M_{j_{0}}} \in \Gamma\left(M_{j_{0}}\right)$, because then $\hat{M}=M_{j_{0}}$ and $\hat{\gamma}=\left.\gamma\right|_{M_{j_{0}}}$ satisfy

$$
\max _{u \in \gamma(\hat{M})} I(u) \leq 2 \pi^{2}+\epsilon \quad \text { and } \quad s(0) \in[0,1] \quad \text { for all } u(t)=(s(t), x(t)) \in \hat{\gamma}(\hat{M})
$$

which is what we are looking for. See [Tan2] for details.

\section{LOCATING CRITICAL POINTS VIA DUAL SETS}

Say that a set $M$ is dual to a homotopy stable class $\mathcal{F}$ with boundary $B$ if

$$
M \cap B=\emptyset \text { and } \quad M \cap A \neq \emptyset \text { for every } A \in \mathcal{F} .
$$

Any sphere $S$ centered at $u_{0}$ with radius $\rho<\left\|u_{0}-u_{1}\right\|$ is obviously dual to the mountain pass class $\mathcal{F}_{u_{0}}^{u_{1}}$. More generally, assume $X$ is a Banach space that splits into $Y \oplus Z$ where $\operatorname{dim}(Y)=n<+\infty$ and consider the class $\mathcal{F}$ of all sets of the form $h\left(B_{Y}\right)$ where $h: B_{Y} \rightarrow X$ is a continuous function on the unit ball of $Y$ which is equal to the identity on the unit sphere $S_{Y}$. Brouwer's theorem easily yields that $Z$ is dual to the class $\mathcal{F}$ which is homotopy stable with boundary $S_{Y}$.

Dual sets are inherently needed in variational settings because, in general, the number $c=c(I, \mathcal{F})$ is unknown and the hypothesis "sup $I(B)<c$ " is not easily verifiable in applications. One usually establishes first the duality between some set $M$ and the family $\mathcal{F}$ which readily implies that $\inf I(M) \leq c$ and then verifies that $\sup I(B)<\inf I(M)$ so as to ensure that the standard boundary condition holds. Systematic use of duality was initiated by Ambrosetti-Rabinowitz in their seminal paper $\mathrm{A}-\mathrm{R}$. The full impact of this notion was recognized by Ghoussoub-Preiss G-P when they showed that "mountain passes" can be found on any separating mountain range $M$ (i.e., critical points can be found on dual sets) provided $\inf I(M)=c$. They then proceeded to demonstrate that judicious choices of dual sets give valuable information on the critical points they contain. The wide ranging applications of duality were later developed by Ghoussoub [G1], G2, who formulated the following form of the min-max principle that involves duality in a fundamental way. It relaxes the boundary condition, weakens the compactness assumption while giving more information about the location of the critical point.

Theorem 3.1 (Locating critical points). Let $G$ be a discrete (or a compact Lie) group acting (differentiably) on a complete connected $C^{1}$-Finsler manifold $X$ without boundary. Let $I$ be a $G$-invariant $C^{1}$-functional on $X$ and consider a $G$ homotopy stable family $\mathcal{F}$ with closed boundary B. Set $c:=c(I, \mathcal{F})$ and let $M$ 
be a closed $G$-subset of $X$ such that:

$$
M \text { is dual to } \mathcal{F} \text { and } \inf I(M) \geq c-\epsilon,
$$

for a small enough $\epsilon>0$. Then, for any $A \in \mathcal{F}$ with $\sup I(A) \leq c+\epsilon$, there exists $x_{\epsilon} \in X$ such that:

(i) $c-\epsilon \leq I\left(x_{\epsilon}\right) \leq c+9 \epsilon$,

(ii) $\left\|I^{\prime}\left(x_{\epsilon}\right)\right\| \leq 18 \sqrt{\epsilon}$,

(iii) $\operatorname{dist}\left(x_{\epsilon}, A\right) \leq \sqrt{\epsilon}$,

(iv) $\operatorname{dist}\left(x_{\epsilon}, M\right) \leq 5 \sqrt{\epsilon}$.

Say that $I$ satisfies $(\mathrm{PS})_{M, c}$, i.e., the Palais-Smale condition around the set $M$ and at level $c$ if a sequence $\left(x_{n}\right)_{n}$ is relatively compact provided it satisfies $\lim _{n} I\left(x_{n}\right)=c, \lim _{n}\left\|I^{\prime}\left(x_{n}\right)\right\|=0$ and $\lim _{n} \operatorname{dist}\left(x_{n}, M\right)=0$. In this case, the above theorem yields a critical point at level $c$ and on the set $M$ provided inf $I(M)=c$. In other words, $K_{c} \cap M \neq \emptyset$ where $K_{c}$ is defined as $K_{c}=\{x \in X ; I(x)=$ $c$ and $\left.I^{\prime}(x)=0\right\}$.

On the surface, Theorem 3.1 looks more restrictive than the classical min-max principle because of the requirement that $\inf _{M} I=c$. However, one needs to notice that the standard boundary condition $\sup I(B)<c=c(I, \mathcal{F})$ simply means that the set $L_{c}:=\{x \in X ; I(x) \geq c\}$ is dual to the class $\mathcal{F}$. The general idea is to find smaller subsets of $L_{c}$ that are still dual to $\mathcal{F}$. For example, the boundary of $L_{c}$ is also dual to $\mathcal{F}$ and the fact that critical points can be found on it means that such points cannot be local minima. Digging a bit more and choosing more elaborate choices for dual sets, one can get additional structural information about variationally generated critical points (See Section 8). But there is much more to this formulation since:

- it helps recover compactness in borderline variational problems by only requesting compactness near dual sets (Section 3.1).

- it helps relax the boundary conditions $\sup I(B)<c=c(I, \mathcal{F})$ to allow equality in many situations (Section 3.2).

- it gives immediate and natural proofs of Ljusternik-Schnirelmann type multiplicity results and in cases involving comparable functionals with common critical levels (Section 4).

- it yields information about the location of critical points which usually translates into qualitative properties of variationally generated solutions of nonlinear equations including Morse-type information (Sections 5.1 and 8.1).

- it leads to the construction of "good paths" (Corollary 3.1 below) and therefore to Taubes' framework for a non-compact Morse theory [Tau3].

Set

$$
K_{c}(\epsilon)=\left\{x \in X ; c-\epsilon<I(x)<c+\epsilon \text { and }\left\|I^{\prime}(x)\right\| \leq \epsilon\right\} .
$$

Corollary 3.1. Let $I$ be a $C^{1}$-functional on $X$ and let $\mathcal{F}$ be a homotopy stable family with boundary $B$ such that $\sup _{B} I<c(I, \mathcal{F})=$ : c. If $I^{\prime}$ is uniformly continuous around $K_{c}(\epsilon)$, then for every $\epsilon>0$, there exist $0<\delta(\epsilon) \leq \epsilon$ and $A \in \mathcal{F}$ such that $\sup _{A} I<c+\delta$ and

$$
A \subseteq I^{c-\delta} \cup K_{c}(\epsilon)
$$


Indeed, otherwise there exists $\epsilon>0$ such that for every $\delta>0$, the set

$$
M_{\delta}:=\{x ; I(x) \geq c-\delta\} \cap\left\{x ;\left\|I^{\prime}(x)\right\| \geq \epsilon\right\}
$$

is dual to $\mathcal{F}$. Theorem 3.1 yields for $\delta$ small enough a sequence $\left(x_{\delta}\right)_{\delta}$ such that $\lim _{\delta \rightarrow 0} I\left(x_{\delta}\right)=c$ and $\lim _{\delta \rightarrow 0} I^{\prime}\left(x_{\delta}\right)=0$ while at the same time approaching the set $M_{\delta}$ and hence the set $\left\{x ;\left\|I^{\prime}(x)\right\| \geq \epsilon\right\}$. This contradicts the uniform continuity assumption on $I$.

3.1. Improved compactness around dual sets. We now exploit duality and Theorem 3.1 to find a second solution for equation (2.14) at a higher energy level. The idea originated in $\mathrm{Tar}$.

Example (4): Sign-changing solutions for a Hardy-Sobolev critical problem. Consider again the boundary value problem

$$
\left\{\begin{aligned}
-\Delta u & =\lambda u+\frac{|u|^{q-2}}{|x|^{s}} u & & \text { in } \Omega \\
u & =0 & & \text { on } \partial \Omega,
\end{aligned}\right.
$$

where $\Omega$ is a smooth bounded domain in $\mathbf{R}^{n}$ containing $0,0<\lambda<\lambda_{1}, 0 \leq s<2$, $n \geq 3$ and $q=2^{*}(s)=\frac{2(n-s)}{n-2}$. A natural class of dual sets are the "Nehari manifolds" defined as

$$
M_{1}:=\left\{u \in H_{0}^{1}(\Omega): u \neq 0 \text { and }\left\langle I_{\lambda}^{\prime}(u), u\right\rangle=0\right\} .
$$

The Sobolev-Hardy inequality and the formulation of $\lambda_{1}$ - in (2.6) - give a constant $c>0$ such that

$$
\left\langle I_{\lambda}^{\prime}(u), u\right\rangle \geq\left(1-\frac{\lambda}{\lambda_{1}}\right)\|u\|_{H_{0}^{1}(\Omega)}^{2}-c\|u\|_{H_{0}^{1}(\Omega)}^{2^{*}(s)}=\|u\|_{H_{0}^{1}(\Omega)}^{2}\left(1-\frac{\lambda}{\lambda_{1}}-c\|u\|_{H_{0}^{1}(\Omega)}^{2^{*}(s)-2}\right) .
$$

This shows that $M_{1}$ is closed and that it is dual to the mountain pass family $\mathcal{F}_{1}$ defined in (2.18). Indeed, fix $\gamma \in \mathcal{F}_{1}$ joining 0 to $v$, where $v \neq 0$ and $I_{\lambda}(v) \leq 0$. Since $\lambda<\lambda_{1}$, we have that $\left\langle I_{\lambda}^{\prime}(\gamma(t)), \gamma(t)\right\rangle>0$ for $t$ close to 0 . On the other hand, since $v \neq 0$, we have that $\left\langle I_{\lambda}^{\prime}(v), v\right\rangle<2 I_{\lambda}(v) \leq 0$. The intermediate value theorem yields a $t_{0}$ such that $\gamma\left(t_{0}\right) \in M_{1}$. This proves the duality and consequently that $c_{1} \geq \inf \left\{I_{\lambda}(u): u \in M_{1}\right\}$. Actually, we have equality since for any $u_{0} \in M_{1}, I_{\lambda}\left(u_{0}\right)$ is the maximum value of $I_{\lambda}$ over the line joining 0 to $u_{0}$. It follows that the solution $u_{1}$ obtained in Proposition 2.5 has minimal energy among all non-trivial solutions; i.e., $c_{1}=I_{\lambda}\left(u_{1}\right)=\inf _{M_{1}} I_{\lambda}$.

To find another solution, let $S_{1}=\left\{u \in H_{0}^{1}(\Omega) ;\|u\|=1\right\}$ be the unit sphere of $H_{0}^{1}(\Omega)$ and consider the family of maps $\mathcal{D}=\left\{h: H_{0}^{1}(\Omega) \rightarrow H_{0}^{1}(\Omega) ; h\right.$ odd homeomorphism\}. Denote by $\Sigma$ the class of all closed symmetric subsets of $S_{1}$. In anticipation of section 4, we define the genus (or Krasnoselski's index) on $\Sigma$ in the following way:

$$
\gamma(A)=\inf \left\{k \text {; there exists } f: A \rightarrow \mathbf{R}^{k} \backslash\{0\} \text { odd and continuous }\right\},
$$

with the usual convention, if no such $k$ exists, or if $A$ is empty. Consider now the class

$$
\mathcal{F}_{2}=\left\{A \subset H_{0}^{1}(\Omega) ; A \text { closed symmetric with } \gamma\left(h(A) \cap S_{1}\right) \geq 2, \forall h \in \mathcal{D}\right\}
$$

which is clearly $\mathbf{Z}_{2}$-homotopy (isotopy) stable. 
Now, for any $u \in L^{2^{*}(s)}(\Omega), u \neq 0$, we let $(v(u), \mu(u))$ be the first (normalized) eigen-pair of the weighted eigenvalue problem:

$$
\left\{\begin{aligned}
-\Delta v-\lambda v & =\mu \frac{|u|^{2^{*}(s)-2}}{|x|^{s}} v & & \text { on } \Omega \\
v & =0 & & \text { on } \partial \Omega .
\end{aligned}\right.
$$

In other words, for each $u \in L^{2^{*}(s)} \backslash\{0\}, v(u)$ is the unique positive solution of (3.7) satisfying $\int_{\Omega} \frac{|u|^{2^{*}(s)-2}}{|x|^{s}} v^{2} d x=1$. Consider the set

$$
M_{2}=M_{1} \cap\left\{u \in H_{0}^{1}(\Omega) ; \int_{\Omega} \frac{|u|^{2^{*}(s)-2}}{|x|^{s}} u v(u) d x=0\right\} .
$$

Proposition 3.1. Suppose $n \geq 6$ and $0<\lambda<\lambda_{1}$; then:

(i) The set $M_{2}$ is dual to the class $\mathcal{F}_{2}$.

(ii) $c_{2}:=c\left(I_{\lambda}, \mathcal{F}_{2}\right)=\inf _{u \in M_{2}} I_{\lambda}(u)<c_{1}+\frac{2-s}{2(n-s)} \mu_{s}^{\frac{n-s}{2-s}}$.

(iii) $I_{\lambda}$ satisfies $(P S)_{M_{2}, c}$ for any $c<c_{1}+\frac{2-s}{2(n-s)} \mu_{s}^{\frac{n-s}{2-s}}$.

Consequently, there is a solution $u_{2}$ for (3.4) satisfying

$$
\int_{\Omega} \frac{\left|u_{2}\right|^{2^{*}(s)-2}}{|x|^{s}} u_{2} v\left(u_{2}\right) d x=0 .
$$

Proof. The uniqueness in the eigenvalue problem (3.7) implies that the map $u \rightarrow$ $v(u)$ is continuous from $L^{2^{*}(s)} \backslash\{0\}$ to $H_{0}^{1}(\Omega)$. To prove that $M_{2}$ is dual to $\mathcal{F}_{2}$, first notice that the map $u \rightarrow \frac{u}{\|u\|}$ defines an odd homeomorphism between $M_{1}$ and the sphere $S_{1}$, so that $\gamma\left(A \cap M_{1}\right) \geq 2$ for every $A \in \mathcal{F}_{2}$. Since the map $h: A \cap M_{1} \rightarrow \mathbf{R}$ given by $h(u)=\int_{\Omega} \frac{|u|^{2^{*}(s)-2}}{|x|^{s}} u v(u) d x$ is odd and continuous, we must have $0 \in h\left(A \cap M_{1}\right)$ which means that $A \cap M_{2} \neq \emptyset$.

For (ii) note that duality implies that $c_{2} \leq \inf _{u \in M_{2}} I_{\lambda}(u)$. To show the reverse inequality, take $u \in M_{2}$, let $v(u)$ be such that $\int_{\Omega} \frac{|u|^{2^{*}(s)-2}}{|x|^{s}} u v(u) d x=0$ and consider an eigenvector $w(u)$ orthogonal to $v(u)$ that corresponds to the "next eigenvalue" of the weighted eigenvalue problem (3.7). It is easy to see that $A=\operatorname{span}\{v(u), w(u)\}$ belongs to $\mathcal{F}_{2}$ and that $I_{\lambda}(u)$ is larger that $\max _{A} I_{\lambda} \geq c_{2}$.

To show that $c_{1} \leq c_{2}<c_{1}+\frac{2-s}{2(n-s)} \mu_{s}^{\frac{n-s}{2-s}}$, it suffices to compute $\sup I_{\lambda}(A)$ where $A$ is the linear subspace spanned by the first solution $u_{1}$ and a truncated extremal function $U_{\epsilon}$ defined in (2.16) where $\epsilon$ is small enough. Again, as in Proposition 2.5, one can use the precise estimates on the truncated extremal functions to show that $c_{2} \leq \sup I_{\lambda}\left(A_{\epsilon}\right)<c_{1}+\frac{2-s}{2(n-s)} \mu_{s}^{\frac{n-s}{2-s}}($ see [G-Y]).

(iii) To show that $I_{\lambda}$ satisfies $(\mathrm{PS})_{M_{2}, c}$ for any $c<c_{1}+\frac{2-s}{2(n-s)} \mu_{s}^{\frac{n-s}{2-s}}$, consider a sequence $u_{k} \in M_{2}$ such that $\lim _{k} I_{\lambda}\left(u_{k}\right)=c$ and $\lim _{k} I_{\lambda}^{\prime}\left(u_{k}\right)=0$. Again $\left(u_{k}\right)_{k}$ is bounded in $H_{0}^{1}(\Omega)$ and hence we can suppose it weakly convergent to some $U^{0} \in H_{0}^{1}(\Omega)$. If it is not convergent, then the set by Proposition 2.4, there exist $r_{k} \rightarrow+\infty$ and a solution $U^{1}$ of

$$
-\Delta U=\frac{|U|^{2^{*}(s)-2}}{|x|^{s}} U \text { on } \mathbf{R}^{n}
$$


such that

$$
\| \nabla\left(u_{k}-U^{0}-r_{k}^{\frac{n-2}{2}} U^{1}\left(r_{k} \cdot\right) \|_{2} \rightarrow 0\right.
$$

and

$$
I_{\lambda}\left(u_{k}\right) \rightarrow I_{\lambda}\left(U^{0}\right)+\frac{2-s}{2(n-s)} \mu_{s}^{\frac{n-s}{2-s}} .
$$

It follows that $I_{\lambda}\left(U^{0}\right)<c_{1}$. However $U^{0}$ is a critical point of $I_{\lambda}$; hence it belongs to $M_{1}$ provided it is not zero. But $\inf I_{\lambda}\left(M_{1}\right)=c_{1}$; hence $U^{0}=0$, and we can therefore suppose that $u_{k} \rightarrow 0$ weakly and $\| \nabla\left(u_{k}-r_{k}^{\frac{n-2}{2}} U^{1}\left(r_{k} \cdot\right) \|_{2} \rightarrow 0\right.$.

Now let $u_{k}^{*}(x)=\left(\frac{1}{r_{k}}\right)^{\frac{n-2}{2}} u_{k}\left(\frac{x}{r_{k}}\right)$ for $x$ in $\Omega_{k}:=\left\{x \in \mathbf{R}^{n} ; \frac{x}{r_{k}} \in \Omega\right\}$. We get from (3.10) that $u_{k}^{*} \rightarrow U^{1}$ strongly. On the other hand, if we consider $v_{k}^{*}(x)=$ $\left(\frac{1}{r_{k}}\right)^{\frac{n-2}{2}} v_{k}\left(\frac{x}{r_{k}}\right)$ for $x \in \Omega_{k}$, where $v_{k}=v\left(u_{k}\right)$, then a weak limit is a function $v^{*}$ that is also a solution of the following eigenvalue problem on $\mathbf{R}^{n}$ :

$$
-\Delta \varphi=\nu \frac{\left|U^{1}\right|^{2^{*}(s)-2}}{|x|^{s}} \varphi \text { in } \mathbf{R}^{n} \text { and } \int_{\mathbf{R}^{n}} \frac{\left|U^{1}\right|^{2^{*}(s)-2}}{|x|^{s}} \varphi^{2} d x<\infty,
$$

while $\int_{\Omega} \frac{\left|U^{1}\right|^{2^{*}(s)-2}}{|x|^{s}} U^{1} v^{*} d x=0$ and $\nu \leq 1$. The contradiction comes from the fact that $U^{1}$ and $v^{*}$ are two orthogonal - yet positive - eigenfunctions (that correspond to a simple eigenvalue). The compactness around $M_{2}$ can also be understood as due to second order conditions that points on $M_{2}$ must satisfy. We shall come back to this aspect of things in Section 8.

To conclude, it suffices to apply Theorem 3.1 to get a critical point $u$ on $M_{2}$. This solution for (3.4) satisfies $\int_{\Omega} \frac{|u|^{2^{*}(s)-2}}{|x|^{s}} u v(u) d x=0$, and therefore it must change sign.

3.2. Relaxing boundary conditions in the presence of dual sets. As mentioned above, one looks for dual sets to guarantee the hypothesis "sup $I(B)<c$ ". Since duality readily implies that inf $I(M) \leq c$, one may substitute it with the more manageable condition "sup $I(B)<\inf I(M)$ ". Theorem 3.1 allows a - sometimes useful - relaxation of this inequality.

Corollary 3.2. Let $G$ be a discrete (or a compact Lie) group acting (differentiably) on a complete connected $C^{1}$-Finsler manifold $X$ without boundary. Let $I$ be a $G$ invariant $C^{1}$-functional on $X$ and consider a $G$-homotopy stable family $\mathcal{F}$ with closed boundary $B$. Let $M$ be a closed $G$-subset of $X$ such that:

$$
M \text { is dual to } \mathcal{F} \text { and } \sup I(B) \leq \inf I(M) \text {. }
$$

If $I$ satisfies $(P S)_{c}$ where $c:=c(I, \mathcal{F})$, then $c$ is a critical value for $I$.

Indeed, duality implies that inf $I(M) \leq c$. So distinguish the two cases: Either $\sup I(B)<c$, which means that Proposition 2.2 applies, or $\sup I(B)=\inf I(M)=$ $c$, which means that $M$ itself verifies the hypothesis of Theorem 3.1. Note that in the second case, a critical point can be found on $M$.

The above corollary is often used in the following context: a standard way for applying the mountain pass theorem (MPT) consists of picking $u_{0}$ and $u_{1}$ such that $I\left(u_{1}\right) \leq I\left(u_{0}\right)$. The boundary $M$ of any neighborhood $V$ of $u_{0}$ not containing $u_{1}$ is then a dual set to $\mathcal{F}_{u_{0}}^{u_{1}}$. This readily implies that $c \geq \inf _{\partial V} I$. If $u_{0}$ is a strict local 
minimum, then $V$ can be chosen in such a way that $I\left(u_{0}\right)<\inf _{\partial V} I$ and we obtain another critical point via the classical MPT. The above corollary allows $u_{0}$ to be a "flat" minimum: $I\left(u_{0}\right)=\inf _{\partial V} I$. In that case, one can obtain a connected set of critical points.

Example (5): Periodic solutions for second order systems. The following example is a less obvious application of Corollary 3.2 and was communicated to us by L. Nirenberg. It is based on the following "linking" situation:

Let $X$ be a Banach space that splits into $Y \oplus Z$ with $\operatorname{dim}(Y)<\infty$. Let $C_{R}=\{u=y+s z ; y \in Y, s \geq 0,\|u\| \leq R\}$ be a half ball in $Y \oplus \mathbf{R} z$ where $z$ is a fixed unit vector in $Z$ such that $I(u) \leq a$ for all $u \in \partial C_{R}$ while $\inf I\left(Z \cap S_{\rho}\right) \geq a$ for some positive $\rho<R$, where $S_{\rho}$ is the sphere centered at 0 of radius $\rho$. Let $\Gamma$ be the class of all continuous functions from $C_{R}$ into $X$ that are extensions of the identity on $\partial C_{R}$. One can use degree theory to show that the set $M=Z \cap S_{\rho}$ is dual to the class $\Gamma$ and therefore Corollary 3.2 applies to yield a critical point for $I$ at a level $c \geq a$. If $c>a$, then $K_{c}$ contains a point which is not a local minimum, and if $c=a$, then $K_{c} \cap Z \cap S_{\rho}=K_{c} \cap M \neq \emptyset$.

Proposition 3.2. Consider a system of $\mathbf{R}^{N}$-valued functions $x(t)$ satisfying

$$
\ddot{x}+V_{x}(t, x)=0
$$

where $V$ is a non-negative real smooth function on $\mathbf{R} \times \mathbf{R}^{N}$ that is periodic in $t$ of period $2 \pi$ and that satisfies for some $\theta\left(0<\theta<\frac{1}{2}\right)$ the following superquadraticity condition:

$$
0<V(t, x) \leq \theta x \cdot V_{x}(t, x) \text { for }|x| \text { large and all } t .
$$

Assume

$$
L:=\max _{\substack{t \\|x| \leq 1}} V(t, x) \leq \frac{3}{2 \pi^{2}} .
$$

Then, (3.13) has a non-constant periodic solution with period $2 \pi$.

The variational expression is given by $I(x)=\int_{0}^{2 \pi}\left[\frac{1}{2}|\dot{x}|^{2}-V(t, x(t))\right] d t$ on the Banach space $X=H^{1}$ of vector-valued $2 \pi$-periodic functions. A critical point $x$ of $I$ is a $2 \pi$-periodic solution of (3.13). For $u \in H^{1}$, write $w=\frac{1}{2 \pi} \int_{0}^{2 \pi} u(t) d t$, $v=u-w$ and equip $H^{1}$ with the following equivalent norm: $\|u\|=\|\dot{v}\|_{2}+\|w\|_{\infty}$. It is easy to check that $I$ is of class $C^{1}$ on $X$ and satisfies the $(P S)_{c}$ condition for any $c$.

To identify a critical level, let $Y$ be the $N$-dimensional subspace of $X$ consisting of the constant vectors and let $Z$ be the space of functions with mean zero. Consider the following $(N+1)$-dimensional subspace of $X$ :

$$
Y_{1}=\left\{x(t)=x_{0}+s e_{1} \cos t ; x_{0} \in Y, s \in \mathbf{R}\right\}
$$

where $e_{1}=(1, \ldots, 0)$, and let $C_{R}$ be the open half-ball

$$
C_{R}=\left\{x(t)=x_{0}+s e_{1} \cos t ;\left|x_{0}\right|<R, 0<s<R\right\}
$$

for some $R$ to be chosen appropriately. For $x$ in $Y_{1}$, we have

$$
I(x)=\frac{1}{2} s^{2} \int_{0}^{2 \pi} \sin ^{2} t d t-\int_{0}^{2 \pi} V(t, x(t)) d t=\frac{\pi}{2} s^{2}-\int_{0}^{2 \pi} V(t, x(t)) d t .
$$


The positivity of $V$ and the superquadraticity condition 3.14 (which implies that $V(t, x) \geq C|x|^{1 / \theta}-C^{\prime}$ for some $C, C^{\prime}>0$ ) easily yield that $I \leq 0$ on $\partial C_{R}$ provided $R$ is large enough. On the other hand, as noted above, the set $M=\{y \in Z ;\|y\|=$

$\left.\sqrt{\frac{6}{\pi}}\right\}$ is dual to the class $\Gamma$ of all continuous functions from $C_{R}$ into $X$ that are extensions of the identity on $\partial C_{R}$. Moreover, if $y \in M$, Sobolev's embedding in one dimension yields that $\|y\|_{\infty} \leq 1$. This combined with assumption (3.15) gives that for $y \in M$,

$$
I(y)=\int_{0}^{2 \pi}\left[\frac{1}{2}|\dot{y}|^{2}-V(t, y(t))\right] d t \geq \frac{3}{\pi}-2 \pi L \geq 0 .
$$

Hence we can use Corollary 3.2 to conclude that $I$ has a non-zero critical point at a level $c \geq \frac{3}{\pi}-2 \pi L \geq 0$. Now if $c>0$, the corresponding critical point must be non-constant since for constant critical points $x$ we have $I(x)=-\int V(t, x) d t \leq 0$. On the other hand, if $c=0$ (which requires that we have equality in (3.15)), then $x$ belongs to the dual set $M$ and therefore cannot be trivial.

\section{Duality And the MUltiplicity of CRITICAL POINTS}

4.1. Group actions and topological indices. A topological index $\operatorname{Ind}_{G}$ associated to a compact group $G$ acting on a manifold $X$ is defined as a mapping from the closed $G$-invariant subsets of $X$ into $\mathbf{N} \cup\{+\infty\}$ that satisfies the following properties:

(I1) $\operatorname{Ind}_{G}(A)=0$ if and only if $A=\emptyset$.

(I2) $\operatorname{Ind}_{G}\left(A_{2}\right) \geq \operatorname{Ind}_{G}\left(A_{1}\right)$ if there is a $G$-equivariant continuous map from $A_{1}$ to $A_{2}$.

(I3) If $F$ is compact invariant, there exists a neighborhood $F^{\delta}=\{x ; \operatorname{dist}(x, F) \leq \delta\}$ of $F$ so that $\operatorname{Ind}_{G}\left(F^{\delta}\right)=\operatorname{Ind}_{G}(F)$.

(I4) $\operatorname{Ind}_{G}\left(A_{1} \cup A_{2}\right) \leq \operatorname{Ind}_{G}\left(A_{1}\right)+\operatorname{Ind}_{G}\left(A_{2}\right)$ for all closed $G$-invariant sets $A_{1}, A_{2}$. The families $\mathcal{F}_{n}=\left\{A ; A\right.$ compact $G$-invariant in $X$ and $\left.\operatorname{Ind}_{G}(A) \geq \mathrm{n}\right\}$ are then clearly G-homotopy stable. When the group action is not free, the set of points with a non-trivial isotropy group may cause problems. So, if we write $\mathcal{I}(G):=$ $\left\{x ; \exists g \neq e, T_{g} x=x\right\}$, then one needs an index that also satisfies:

(I5) If $F$ is compact invariant with $F \cap \mathcal{I}(G)=\emptyset$, then $\operatorname{Ind}_{G}(F)<+\infty$ and $F$ contains at least $\operatorname{Ind}_{G}(F)$ orbits.

There are many examples of topological indices associated with the classical groups $\mathbf{Z}_{p}$ and $S^{1}$. Other cohomological indices associated with more general Lie groups were introduced by Faddell, Husseini, Rabinowitz and others (see for example F-H-R .

$\mathbf{Z}_{2}$-indices: We have already defined (in Section 3) the "cohomotopic index" of Krasnoselski. A "homological" $\mathbf{Z}_{2}$-index with additional remarkable properties is the mod 2-genus considered by Coffman [Co1]. Let $P^{N}=\left\{(x,-x): x \in S^{N}\right\}$ be the $\mathrm{N}$-dimensional real projective space. The homology groups (with $\mathbf{Z}_{2}$ coefficients) of $P^{N}$ are then $H_{k}\left(P^{N}\right)=\mathbf{Z}_{2}$ if $k \leq N$ and $H_{k}\left(P^{N}\right)=\{0\}$ if $k>N$. For a compact symmetric subset $A$ of $S^{\bar{N}}$, define the mod 2-genus $\gamma_{2}$ by $\gamma_{2}(A)=$ $\sup \left\{k ;\left(i_{*}\right)_{k-1} \neq 0\right\}$ where $i: \tilde{A}=\{(x,-x): x \in A\} \rightarrow P^{N}$ is the natural injection and $\left(i_{*}\right)_{k}: H_{k}(\tilde{A}) \rightarrow H_{k}\left(P^{N}\right)$ is the corresponding homomorphism between the $k$-dimensional homology groups. The set function $\gamma_{2}$ enjoys properties (I1)-(I5) listed above. It also satisfies the following: 
(I6) $\gamma_{2}\left(A_{1} \cap A_{2}\right) \geq \gamma_{2}\left(A_{1}\right)+\gamma\left(A_{2}\right)-N-1$ for all closed symmetric subsets $A_{1}, A_{2}$. The classes $\mathcal{F}_{n}=\left\{A ; A\right.$ closed symmetric with $\left.\gamma_{2}(A) \geq n\right\}$ are $\mathbf{Z}_{2}$-homotopy stable families and Property (I6) implies that for each $k(1 \leq k \leq N+1)$, the families $\mathcal{F}_{k}$ and $\mathcal{F}_{N-k+2}$ are dual to each other.

$S^{1}$-indices: Let $E=S^{\infty}$ be the classifying space for the group $S^{1}$. The cohomology ring (with rational coefficients) of $\mathbf{C P}^{\infty}=E / S^{1}$ is then generated by an element $\omega_{2}$ of degree 2 , in such a way that $H^{2 k}\left(\mathbf{C P}^{\infty}\right)=\mathbf{Q} \omega_{2}^{k}$ and $H^{2 k+1}\left(\mathbf{C P}^{\infty}\right)=$ $\{0\}$. Suppose now $X$ is a manifold with a free $S^{1}$-action. For any $S^{1}$-invariant compact subset $A$, define the index

$$
\operatorname{Ind}_{S^{1}}(A)=\sup \left\{k ; \tilde{f}^{*}\left(\omega_{2}\right)^{k-1} \neq 0\right\}
$$

where $f: A \rightarrow E$ is a classifying map, $\tilde{f}: A / S^{1} \rightarrow E / S^{1}$ is the map it induces on the orbit-space, and $\tilde{f}^{*}: H^{*}\left(\mathbf{C P}^{\infty}\right) \rightarrow H^{*}\left(A / S^{1}\right)$ is the corresponding homeomorphism between the cohomology algebras. Ind $_{S^{1}}$ enjoys the properties of an index listed above. Again, $\mathcal{F}_{n}=\left\{A ; A\right.$ compact invariant with $\left.\operatorname{Ind}_{S^{1}}(A) \geq n\right\}$ is an $S^{1}$-homotopy stable family.

\subsection{Multiplicity results à la Ljusternik-Schnirelmann.}

Corollary 4.1. Let $G$ be a discrete (or a compact Lie) group acting (differentiably) on a complete connected $C^{1}$-Finsler manifold $X$ and suppose $\operatorname{Ind}_{G}$ is an index associated to $G$. Let $I$ be a $G$-invariant $C^{1}$-functional satisfying $(P S)_{c}$ for every c. For each $n$, let $c_{n}=c\left(I, \mathcal{F}_{n}\right)$ where $\mathcal{F}_{n}=\{A ; A$ compact $G$-invariant in $X$ and $\left.\operatorname{Ind}_{G}(A) \geq n\right\}$. If $-\infty<c_{j}=c_{j+p}<+\infty$ and $K_{c_{j}} \cap \mathcal{I}(G)=\emptyset$, then $\operatorname{Ind}_{G}\left(K_{c_{j}}\right) \geq$ $p+1$.

Proof. By (I2), the families $\mathcal{F}_{n}$ are $G$-homotopy stable. Assume that $c_{j}=c_{j+p}$ but $\operatorname{Ind}_{G}\left(K_{c_{j}}\right) \leq p$. Use (I3) to find a neighborhood $U$ of $K_{c_{j}}$ such that $\bar{U} \cap \mathcal{I}(G)=\emptyset$ and $\operatorname{Ind}_{G}(\bar{U}) \leq p$. For every $A$ in $\mathcal{F}_{j+p}$, we have by (I4) that $A \backslash U \in \mathcal{F}_{j}$. Hence $(A \backslash U) \cap\left\{I \geq c_{j}\right\} \neq \emptyset$. It follows that $M:=\left\{I \geq c_{j+p}\right\} \backslash U=\left\{I \geq c_{j}\right\} \backslash U$ is dual to $\mathcal{F}_{j+p}$. By Theorem 3.1, the set $K_{c_{j}} \backslash U=K_{c_{j+p}} \cap M$ is then non-empty, contradicting that $K_{c_{j}} \subset U$.

Example (6): Multiple closed trajectories for Hamiltonian systems. Consider a $2 n$-dimensional linear symplectic space. This is equivalent to choosing an antisymmetric matrix $J \in \mathcal{L}\left(\mathbf{R}^{2 n}\right)$ such that $J^{2}=-I$. Given a compact smooth hypersurface $\Sigma \subset \mathbf{R}^{2 n}$, define $n(x)$ to be the exterior normal to $\Sigma$ at $x$. Since $J$ is antisymmetric, $J n(x)$ tangent to $\Sigma$ at $x$, and the equation $\dot{x}=J n(x)$ defines the symplectic flow on $\Sigma$. We are interested in finding and studying closed trajectories of the symplectic flow. Existence was first proved by Rabinowitz [R3] under the additional assumption that $\Sigma$ is star-shaped with respect to the origin, and multiplicity by Ekeland and Lasry [E-L] under more stringent conditions on $\Sigma$ (see also [E2]).

Theorem 4.1. Assume $\Sigma$ bounds a convex set in such a way that for some $r>0$, we have $r<\|x\|<r \sqrt{2}$ for all $x \in \Sigma$. Then $\Sigma$ carries at least $n$ distinct closed trajectories of the symplectic flow.

We first write a suitable variational formulation. Choose $\beta>2$, and let $\alpha \in(1,2)$ be its conjugate exponent. Let $G: \mathbf{R}^{2 n} \mapsto \mathbf{R}$ be a non-negative strictly convex $C^{1}$ function such that $G(0)=0, G(x)>0$ for $x \neq 0$ and $G(s x)=s^{\beta} G(x)$ for all $s>0$ 
and all $x$. Consider the closed subspace $V \subset L^{\beta}\left(S^{1} ; \mathbf{R}^{2 n}\right)$ consisting of all functions $v$ with mean zero and the compact linear map $\Pi: V \mapsto C\left(S^{1}, \mathbb{R}^{2 n}\right)$ defined by $[\Pi(v)](t)=\int_{0}^{t} v(s) d s, \int_{0}^{1}(\Pi v) d t=0$. Define a $C^{1}$-functional $I$ on $V$ by:

$$
I(v)=\int_{0}^{1} \frac{1}{2}(J v, \Pi v) d t+\int_{0}^{1} G(-v) d t .
$$

A critical point $v$ of $I$ satisfies the equation:

$$
J \Pi v(t)+x_{0}=G^{\prime}(v(t)) \text { a.e. }
$$

where $x_{0} \in \mathbf{R}^{2 n}$ is some constant vector. Standard convexity arguments yield that the inverse of $G^{\prime}: \mathbf{R}^{2 n} \mapsto \mathbf{R}^{2 n}$ is $H^{\prime}$, where $H: \mathbf{R}^{2 n} \mapsto \mathbf{R}$ is the Legendre-Fenchel transform of $G$, i.e., $H(x):=\max \left\{x y-G(y) ; y \in \mathbf{R}^{2 n}\right\}$. In terms of $H$, equation (4.1) becomes:

$$
v(t)=\frac{d}{d t}\left(\Pi v+x_{0}\right)=H^{\prime}\left(\Pi v(t)+x_{0}\right) \text { a.e. }
$$

so that $x(t):=\Pi v(t)+x_{0}$ is a 1-periodic solution of the Hamiltonian system:

$$
\dot{x}=J H^{\prime}(x) .
$$

It is clear that $H$ itself is a first integral of equation (4.2), that is, $H(x(t))=h$ for some constant $h>0$, and $x(t)$ is a closed trajectory of the symplectic flow on the level set $H=h$. The function $H$ satisfies properties similar to those of $G$ : it is positive at every point except 0 , where it vanishes; it is convex and $C^{1}$, and positively homogeneous of degree $\alpha$. All its level sets are homothetic to each other. Defining:

$$
\Sigma=\left\{x \in \mathbf{R}^{2 n} \mid H(x)=1\right\},
$$

we find that $y(t):=h^{-1 / \alpha} x\left(h^{\frac{(2-\alpha)}{\alpha}} t\right)$ is a closed trajectory of the symplectic flow on $\Sigma$.

Conversely, with any compact, convex hypersurface $\Sigma$ surrounding the origin, one can associate a function $G$, satisfying the above assumptions and such that (4.3) holds. Any periodic solution of equation (4.2) lying on $\Sigma$ then gives rise by time-rescaling to a closed trajectory of the symplectic flow on $\Sigma$.

We now show that the functional $I$ does indeed have critical points. The assumption on $\Sigma$ implies that $G_{r} \leq G \leq G_{R}$, where $G_{\rho}(x)=\frac{\alpha-1}{\alpha}\left(\frac{\rho^{\alpha}}{\alpha}\right)^{\frac{1}{\alpha-1}}\|x\|^{\beta}$. Hence, with obvious notations, $I_{r} \leq I \leq I_{R}$. The functions $I_{r}, I$ and $I_{R}$ all satisfy the Palais-Smale condition on $V$. Moreover, the minima of $I_{r}$ and $I_{R}$ can be computed explicitly, yielding the following estimate on the minimum of $I$ :

$$
-\left(1-\frac{\alpha}{2}\right)\left(\frac{\alpha}{2 \pi r^{2}}\right)^{\frac{\alpha}{2-\alpha}} \leq \min I \leq-\left(1-\frac{\alpha}{2}\right)\left(\frac{\alpha}{2 \pi R^{2}}\right)^{\frac{\alpha}{2-\alpha}} .
$$

We now use the $S^{1}$-invariance of the functional $I$ to get the multiplicity result. More precisely, the space $V$ is endowed with a natural $S^{1}$ action via the formula $T_{\theta} v(t)=v(t+\theta)$, in such a way that $I(\theta v)=I(v)$ for all $\theta \in S^{1}$ and $v \in V$. This action is not free: all $1 / k$-periodic functions in $V$ are $k$-periodic points of the action. Further computations, using the homogeneity of $G$, show that if $v \in V$ is $k$-periodic, then

$$
I(v) \geq a_{k}:=-\left(1-\frac{\alpha}{2}\right)\left(\frac{\alpha}{2 k \pi r^{2}}\right)^{\frac{\alpha}{2-\alpha}} .
$$


It follows that the $S^{1}$-action is free on the sublevel $I^{a_{2}}=\left\{v \in V ; I(v)<a_{2}\right\}$. Since $a_{2} \geq a_{1}=\min I$, this set is non-empty. In addition, there is an $S^{1}$-equivariant map $\varphi$ of the sphere $S^{2 n-1}$ with the standard $S^{1}$-action into $I^{a_{2}}$ : for $\xi \in S^{2 n-1}$, $v=\varphi(\xi) \in V$ is the function defined by $v(t)=\rho e^{2 \pi J t} \xi$ and the constant $\rho$ can be chosen so that $\varphi\left(S^{2 n-1}\right) \in I^{a_{2}}$. Clearly $\varphi\left(S^{2 n-1}\right)$ has $S^{1}$-index $n$, so that $\operatorname{Ind}_{S^{1}}\left(I^{a_{2}}\right) \geq n$, and we may apply Corollary 4.1 to get at least $n$ distinct critical points of $I$ in $I^{a_{2}}$ which lie on pairwise distinct $S^{1}$-orbits.

We now translate these results in geometrical terms. Two different functions $v_{1}$ and $v_{2}$ in $V$ correspond to the same closed trajectory in $\Sigma$ iff $v_{2}(t)=$ $v_{1}(k(t+\theta))$ for some $\theta \in S^{1}, k \in \mathbf{N}$. Since $v_{1}$ and $v_{2} \in I^{a_{2}}$, both have minimal period 1. It follows that $k=1$, and we must have $v_{2}=T_{\theta} v_{1}$ for some $\theta$, that is, if $v_{1}$ and $v_{2}$ belong to the same orbit of the $S^{1}$ action in $V$. So $n$ pairwise distinct $S^{1}$-orbits correspond to $n$ different closed trajectories, and the theorem is proved.

4.3. Comparable functions and other types of multiplicity. It is clear that modulo compactness conditions - two comparable functionals with the same finite infimum must share a common critical point, namely a minimum. R. Coffman Co2 used that observation to find multiple non-radial solutions for semi-linear equations on expanding annuli. When the non-linearity is non-homogeneous, constraint minimization methods are not applicable and have to be replaced by global min-max methods. The following useful application of Theorem 3.1 allowed Suzuki [Su] to extend Coffman's result to more general non-linearities.

Corollary 4.2. Let I be a $C^{1}$-functional on a complete connected $C^{1}$-Finsler manifold $X$ and consider a homotopy stable family $\mathcal{F}$ of compact subsets of $X$ with a closed boundary B. Assume I satisfies $(P S)_{c}$ where $c=c(I, \mathcal{F})$. Let $J$ be another continuous functional such that $J \leq I$ on $X$, while $c(J, \mathcal{F})=c$ and $\sup J(B)<c$. Then, there exists $x \in X$ such that $I^{\prime}(x)=J^{\prime}(x)=0$ and $I(x)=J(x)=c$.

Indeed, it is enough to realize that the set $M=\{J \geq c\}$ is then dual to $\mathcal{F}$ while $\inf I(M)=c$. Theorem 3.1 then yields the claimed result.

Example (7): Non-radial solutions on the annulus. For $a>0$, let $\Omega_{a}=$ $\{x ; a<|x|<a+1\} \subset \mathbf{R}^{2}$ be the corresponding 2-dimensional annulus and consider the equation

$$
\left\{\begin{array}{cl}
-\Delta u=f(u) & \text { if } x \in \Omega_{a} \\
u>0 & \text { if } x \in \Omega_{a} \\
u=0 & \text { if } x \in \partial \Omega_{a},
\end{array}\right.
$$

where $f: \mathbf{R} \rightarrow \mathbf{R}$ is a continuous function with $f(0)=0$. Let $F(s)=\int_{0}^{s} f(t) d t$.

Proposition 4.1. Suppose that $f$ verifies the following conditions for some $\sigma>0$ and $K>0$ :

$$
0 \leq f(u) \leq K u^{p} \quad \text { where } p>1,
$$

and

$$
F(u) \leq\left(\frac{1}{2}-\sigma\right) f(u) u,
$$

for all $u>0$. Then, for any integer $N$ and for sufficiently large a, problem (4.4) admits $N$ distinct non-radial solutions. 
Let $X^{a}=H_{0}^{1}\left(\Omega_{a}\right)$ and consider the $C^{1}$ - functional $I_{a}: X^{a} \rightarrow \mathbf{R}$ defined by

$$
I_{a}(v)=\int_{\Omega_{a}}\left(\frac{1}{2}|\nabla v|^{2}-F\left(v^{+}\right)\right) d x .
$$

$I_{a}$ verifies $(P S)_{c}$ for every $c$, and each nonzero critical point $u \in X^{a}$ of $I_{a}$ is a classical solution for (4.4). Let $\sum_{a}$ denote the solution set of (4.4) and write $\sum_{a}^{0}=\left\{u \in \sum_{a} ; u=u(|x|)\right\}$ for the set of radial solutions.

Write $x=\left(x_{1}, x_{2}\right)=(r \cos \theta, r \sin \theta)$ in polar coordinates. For $\ell=1,2, \ldots$ denote by $T_{\ell}$ the rotation by $\frac{2 \pi}{l}$

$$
T_{\ell}\left(x_{1}, x_{2}\right)=\left(r \cos \left(\theta+\frac{2 \pi}{\ell}\right), r \sin \left(\theta+\frac{2 \pi}{\ell}\right)\right)
$$

and consider the groups $G_{\ell}=\left\{T_{\ell}^{j} ; 0 \leq j \leq \ell-1\right\} \subset O(2)$ acting on $\Omega_{a}$. We write $X_{\ell}^{a}$ for the subspace of $X^{a}$ consisting of the $G_{\ell}$-invariant functions.

Introduce the transformation $S_{\ell}: \Omega_{a} \rightarrow \Omega_{a}$ by $S_{\ell} x=(r \cos \ell \theta, r \sin \ell \theta)$. It induces a bijective mapping $S_{\ell}^{*}: X^{a} \rightarrow X^{a}$. For $w \in X^{a}$ we define $I_{a, \ell}(w)=$ $I_{a}\left(S_{\ell}^{*-1} w\right)$.

The hypothesis allows us to set up the mountain pass theorem for $I_{a, \ell}$ for each $a>0$ and each integer $\ell$. Indeed, for $e \in X^{a}$, write

$$
\Gamma^{a}(e)=\left\{\gamma \in C\left([0,1] ; X^{a}\right) ; \gamma(0)=0, \gamma(1)=e\right\},
$$

and $c_{\ell}(a):=c\left(I_{a, \ell} ; \Gamma^{a}(e)\right)=\inf _{\gamma \in \Gamma(e)} \max _{0 \leq t \leq 1} I_{a, \ell}(\gamma(t))$.

Claim. The following properties hold for every $a>0$ :

(i) $I_{a, 1} \leq I_{a, 2} \leq \ldots \leq I_{a, \ell} \leq \ldots$.

(ii) If $e \in X^{a}$ is such that $I_{a, \ell}(e)<0$ and $c_{\ell}(a)>0$ for $1 \leq \ell \leq N$, then each $c_{\ell}(a)$ is a critical value for $I_{a, \ell}$ and $c_{1}(a) \leq c_{2}(a) \leq \ldots \leq c_{N}(a)$.

(iii) If $c_{\ell}(a)=c_{\ell-1}(a)$, then $c_{\ell}(a) \in I_{a}\left(\sum_{a}^{0}\right)$.

Indeed, note that

$$
I_{a, \ell}(w)=\int_{0}^{2 \pi} d \theta \int_{a}^{a+1}\left\{\frac{1}{2}\left(w_{r}^{2}+\ell^{2} r^{-2} w_{\theta}^{2}-F\left(w^{+}\right)\right\} r d r\right.
$$

which clearly implies (i). (ii) is a consequence of the mountain pass theorem and the fact that $I_{a, \ell}$ verifies $(P S)_{c}$ for any $c$. For (iii) we note that if $c_{\ell}(a)=c_{\ell-1}(a)$, then by Corollary 4.2, there exists $w \in X^{a}$ such that $\left(I_{a, \ell}\right)^{\prime}(w)=0, I_{a, \ell}(w)=c_{\ell}(a)$ and $I_{a, \ell}(w)=I_{a, \ell-1}(w)$. But the last relation implies that $w_{\theta} \equiv 0$ by $(4.7)$, and hence $w \in \sum_{a}^{0}$. In other words $c_{\ell}(a) \in I^{a}\left(\sum_{a}^{0}\right)$.

The rest of the proof of Proposition 4.1 consists of showing that, for a large enough $a$, we have that $c_{1}(a), c_{2}(a), \ldots, c_{\ell}(a) \notin I_{a}\left(\sum_{a}^{0}\right)$ and therefore the critical levels are necessarily distinct: $c_{1}(a)<c_{2}(a)<\ldots<c_{\ell}(a)<\ldots$. To do that, one needs to analyze the asymptotic behaviour of $c(a)$ as $a \rightarrow+\infty$. Actually one can show that whenever a family $\left\{u_{a}\right\}_{a \gg 1}$ with $u_{a} \in \sum_{a}^{0}$ is such that $I_{a}\left(u_{a}\right)$ is bounded as $a \rightarrow \infty$, then $I_{a}\left(u_{a}\right) \rightarrow 0$ as $a \rightarrow \infty$. This would yield the required contradiction. See $[\mathrm{Su}]$ for details.

\section{MORSE INDICES OF VARIATIONALLY GENERATED CRITICAL POINTS}

The topological properties of a homotopy stable class of sets relate to the Morse indices of the critical points obtained by min-maxing over that class. We assume that the group $G$ which acts on the manifold under study $X$ also acts on $\mathbf{R}^{k}$ as 
well as on the standard $k$-dimensional Euclidian sphere $S^{k}$. If $D$ and $E$ are two topological spaces on which $G$ acts continuously, we denote by $C_{G}(D ; E)$ the set of all continuous and $G$-equivariant maps from $D$ into $E$. $G$-invariant sets will sometimes be called $G$-sets. $B$ is for now a fixed closed $G$-subset of the manifold $X$. We introduce the following notions.

Definition 5.1. (1) A family $\mathcal{F}$ of $G$-subsets of $X$ is said to be $G$-homotopic of dimension $n$ with boundary $B$ if there exists a compact $G$-subset $D$ of $\mathbf{R}^{n}$ containing a closed subset $D_{0}$ and a continuous $G$-invariant function $\sigma$ from $D_{0}$ onto $B$ such that:

$$
\mathcal{F}=\left\{A \subset X ; A=f(D) \text { for some } f \in C_{G}(D ; X) \text { with } f=\sigma \text { on } D_{0}\right\} .
$$

(2) Dually, we can introduce the cohomotopic classes by considering a fixed $G$ equivariant continuous map $\sigma^{*}: B \rightarrow S^{k}$ and by defining for any closed $G$-subset $A$ of $X$ containing $B$, the "index":

$$
\gamma_{G}\left(A ; B, \sigma^{*}\right)=\inf \left\{n ; \exists f \in C_{G}\left(A ; S^{n}\right) \text { with } f=\sigma^{*} \text { on } B\right\} .
$$

A family $\mathcal{F}$ of subsets of $X$ is said to be $G$-cohomotopic of dimension $n$ with boundary $B$ if there exists a continuous and $G$-equivariant $\sigma^{*}: B \rightarrow S^{n}$ such that

$$
\mathcal{F}=\left\{A ; A \text { compact } G \text {-subset of } X, A \supset B \text { and } \gamma_{G}\left(A ; B, \sigma^{*}\right) \geq n\right\} .
$$

(3) A family $\mathcal{F}$ of $G$-subsets of $X$ is said to be a homological family of dimension $n$ with boundary $B$ if for some non-trivial class $\alpha$ in the $n$-dimensional relative homology group $H_{n}(X, B)$ we have that

$$
\mathcal{F}=: \mathcal{F}(\alpha)=\left\{A ; A \text { compact } G \text {-subset of } X, A \supset B \text { and } \alpha \in \operatorname{Im}\left(i_{*}^{A}\right)\right\}
$$

where $i_{*}^{A}$ is the homomorphism $i_{*}^{A}: H_{n}(A, B) \rightarrow H_{n}(X, B)$ induced by the immersion $i: A \rightarrow X$. Here, we use singular homology with rational or real coefficients.

For simplicity, we deal only with the following kind of - possibly degenerate critical points. Assume $v_{0}$ is a critical point of the $C^{2}$-functional $I$ in such a way that $I^{\prime \prime}\left(v_{0}\right)$ is a Fredholm operator on its Hilbertian domain $E$. Then $E=E_{0} \oplus E_{1}$ where $E_{0}$ and $E_{1}$ denote the kernel and range of $I^{\prime \prime}\left(v_{0}\right)$ respectively. Moreover, the dimension of $E_{0}$ is finite and $I^{\prime \prime}\left(v_{0}\right)$ is invertible on $E_{1}$. From basic spectral theory, it follows that $E_{1}=E_{+} \oplus E_{-}$where $E_{+}$and $E_{-}$are the positive and negative spaces associated with $I^{\prime \prime}\left(v_{0}\right)$ restricted to $E_{1}$. The Morse index of $v_{0}$ is defined then as $m\left(v_{0}\right)=\operatorname{dim}\left(E_{-}\right)$and the augmented Morse index of $v_{0}$ as $m^{*}\left(v_{0}\right)=\operatorname{dim}\left(E_{0}\right)+\operatorname{dim}\left(E_{-}\right)$.

The following theorem gives one-sided relations between the Morse index and the homotopic (resp. cohomotopic) dimension of the class, while for homological families, two-sided estimates are available. These results have been established in various levels of generality by [L-S], [So], Ba-L1], G2], Tan1], $\mathrm{V}]$.

Theorem 5.1. Let $G$ be a discrete (or compact Lie) group acting freely (and differentiably) on a complete $C^{2}$-Riemannian manifold $X$. Let $I$ be a $G$-invariant $C^{2}$-functional on $X$ and let $\mathcal{F}$ be a $G$-homotopy stable with boundary $B$. Assume that $I$ verifies $(P S)_{c}$ where $c:=c(I, \mathcal{F})$, that $I^{\prime \prime}(x)$ is Fredholm for each $x$ at level $c$ and that $\sup _{B} I<c$.

(i) If $\mathcal{F}$ is $G$-homotopic of dimension $n$, then there is $x \in K_{c}$ with $m(x) \leq n$.

(ii) If $\mathcal{F}$ is $G$-cohomotopic of dimension n, then there is $x \in K_{c}$ with $m^{*}(x) \geq n$. 
(iii) If $\mathcal{F}$ is $G$-homological of dimension $n$, then there is $x \in K_{c}$ with $m(x) \leq n \leq$ $m^{*}(x)$.

5.1. Distinguishing solutions via their Morse indices. One of the main applications of the above considerations has been existence results for closed trajectories of the symplectic flow on convex compact hypersurfaces in $\mathbf{R}^{2 n}$. As we pointed out in Example (5), there is a variational setting for that geometric problem, but a single closed trajectory of the symplectic flow corresponds to an infinite sequence of critical points of the associated functional. Each of these critical points is characterized by an integer $k \geq 1$ and a $\theta \in \mathbf{R} / \mathbf{Z}$, where $k$ is the number of times one runs around the fundamental trajectory and $\theta$ is the phase shift. In order to find solutions with a prescribed minimal period, one could use the above theorem to look for critical points of Morse index at most one. Unfortunately, the relevant functional is not always $C^{2}$ and therefore standard Morse theory does not apply. We remedy the situation in Section 10, by presenting a non-smooth version of Theorem 5.1.

Multiplicity results also require ways to distinguish variationally generated critical points. In Theorem 4.1, this difficulty was overcome by energy estimates, thanks to the pinching condition. The use of Morse indices for such a task was initiated by Ekeland [E0], who showed that there are infinitely many symplectic closed trajectories on a strictly convex hypersurface provided they are all non-resonant (in a precise sense involving only the Liapounov exponents and the action along each trajectory). Later on, Ekeland-Lassoued [E-L] and then Ekeland-Hofer [E-H2] proved that any compact strictly convex hypersurface in $\mathbf{R}^{2 n}$ carries at least two closed symplectic trajectories. The best results to date are due to Y. Long and his students. In [L-Z, they proved that any such hypersurface carries at least $[n / 2]+1$ closed trajectories, while in [L-L-Z, they show that there are at least $n$ if, in addition, one assumes symmetry of the surface around the origin. Unfortunately, we cannot go into the proofs of these beautiful results here and the reader is referred to [E2], [Lo1] and [Lo2] for that story. Instead, we present a very simple example to illustrate the use of Theorem 5.1.

Example (8): An asymptotically linear non-resonant problem. Consider the following asymptotically linear non-resonant boundary value problem:

$$
\left\{\begin{aligned}
-\Delta u-\lambda u & =g(u) & & \text { on } \Omega \\
u & =0 & & \text { on } \partial \Omega
\end{aligned}\right.
$$

where $\Omega \subset \mathbf{R}^{n}$ is a smoothly bounded domain. Denote by $\left(\lambda_{i}\right)_{i \geq 1}$ the eigenvalues of $-\Delta$ on $H_{0}^{1}(\Omega)$.

Proposition 5.1. Let $g$ be a bounded real $C^{1}$-function such that $g(0)=0$. Assume that $\lambda \in\left(\lambda_{k}, \lambda_{k+1}\right)$ and that $g^{\prime}(0)+\lambda \in \mathbf{R} \backslash\left(\lambda_{k}, \lambda_{k+1}\right)$. Then, problem (5.1) has at least one non-trivial solution.

To get a non-trivial solution, it suffices - in view of the hypothesis - to find a critical point $u$ with Morse index equal to $k$ for the functional

$$
I(u)=\frac{1}{2} \int_{\Omega}\left(|\nabla u|^{2}-\lambda|u|^{2}\right) d x-\int_{\Omega} G(u) d x
$$

on $X=H_{0}^{1}$, where $\mathrm{G}$ denotes a primitive of $\mathrm{g}$. To do that, let $Y$ be the subspace of $H_{0}^{1}$ spanned by the eigenfunctions corresponding to the eigenvalues $\left(\lambda_{i}\right)_{i \leq k}$ and let $Z$ be its linear supplement. For $R$ large enough, we have $\sup I\left(S_{Y}(R)\right)<\inf I(Z)$. Now regard $\sigma=\left[S_{Y}\right]$ as the generator of the homology $H_{k-1}\left(S_{Y}, \emptyset\right)$ and let $\beta \in$ 
$H_{k}\left(X, S_{Y}\right)$ be such that $\partial \beta=\sigma$. Consider $\mathcal{F}=\mathcal{F}(\beta)$ to be the corresponding homological family. Since $\sigma \neq 0$ in $H_{k-1}(X \backslash Z)$, it follows that $Z$ is dual to $\mathcal{F}$ and $\sup I\left(S_{Y}(R)\right)<\inf I(Z) \leq c(I, \mathcal{F})$. The rest follows from Theorem 5.1(iii).

5.2. Morse indices and energy estimates. Again let $\Omega$ be a smoothly bounded domain in $\mathbf{R}^{n}(n \geq 3)$ and let $f: \Omega \times \mathbf{R} \rightarrow \mathbf{R}$ be a continuous function with primitive $F(\cdot, t)=\int_{0}^{t} f(\cdot, s) d s$. Consider the $C^{1}$-functional

$$
I(u)=\frac{1}{2} \int_{\Omega}|\nabla u|^{2} d x-\int_{\Omega} F(x, u) d x
$$

on $H_{0}^{1}(\Omega)$ whose critical points are the weak solutions of

$$
\left\{\begin{aligned}
-\Delta u & =f(x, u) & & \text { on } \Omega \\
u & =0 & & \text { on } \partial \Omega .
\end{aligned}\right.
$$

Let $\left(E_{k}\right)_{k=1}^{\infty}$ be an increasing sequence of subspaces of $H_{0}^{1}(\Omega)$ such that $\operatorname{dim} E_{k}=k$ and $\overline{\cup_{k=1}^{\infty} E_{k}}=E:=H_{0}^{1}(\Omega)$, and define the min-max values $c_{k}=\inf _{h \in \mathcal{H}_{k}} \sup _{h\left(E_{k}\right)} I$, where

$$
\mathcal{H}_{k}=\left\{h \in C(E, E) ; h \text { is odd and } h(v)=v \text { for }\|v\|>R_{k} \text { for some } R_{k}>0\right\} .
$$

We now describe how the Morse indices of variationally obtained solutions of (5.3) lead to estimates on the growth of their corresponding critical levels. This was observed by Bahri-Lions [Ba-L2]. (See also Tanaka Tan1].)

Proposition 5.2. Suppose that for some $p>1,|F(x, s)| \leq A+B|s|^{p+1}$ for almost every $x \in \Omega$ and all $s \in \mathbf{R}$ where $A, B$ are non-negative constants.

(1) If $n \geq 3$ and $p<\frac{n+2}{n-2}$, there are constants $C, D>0$ such that $c_{k} \geq$ $C k^{\frac{p+1}{p-1} \frac{2}{n}}-D$.

(2) If $n=2$, then for any $\epsilon>0$ there are constants $C_{\epsilon}, D_{\epsilon}>0$ such that

$$
c_{k} \geq C_{\epsilon} k^{\frac{p+1}{p-1}-\epsilon}-D_{\epsilon} .
$$

From the hypothesis on $f$, we have

$$
I(u) \geq \frac{1}{2}\|\nabla u\|_{2}^{2}-\int_{\Omega} F(x, u) d x \geq \frac{1}{2}\|\nabla u\|_{2}^{2}-B\|u\|_{p+1}^{p+1}-A .
$$

Set $K(u)=\frac{1}{2}\|\nabla u\|_{2}^{2}-B\|u\|_{p+1}^{p+1}$ on $H_{0}^{1}(\Omega)$ and define the min-max values $b_{k}=$ $\inf _{h \in \mathcal{H}} \sup K$, so that $c_{k} \geq b_{k}-A$. To estimate $b_{k}$, one identifies a cohomotopic $h \in \mathcal{H}_{k} h\left(E_{k}\right)$

family $\mathcal{F}$ of dimension $k$ in such a way that if $D_{k}$ denotes the ball in $E_{k}$ of radius $R_{k}$ and if $\gamma \in \mathcal{H}_{k}$, then $\gamma\left(D_{k}\right) \in \mathcal{F}$. It follows from Theorem 5.1 that there is a $v_{k} \in H_{0}^{1}(\Omega)$ such that $K\left(v_{k}\right) \leq b_{k}, K^{\prime}\left(v_{k}\right)=0$ and $m^{*}\left(v_{k}\right) \geq k$. Since

$$
K^{\prime \prime}(v)(h, h)=\left(\left(-\Delta-B p(p+1)|v|^{p-1}\right) h, h\right) \text { for all } v,
$$

where $(\cdot, \cdot)$ denotes the duality product between $H^{-1}(\Omega)$ and $H_{0}^{1}(\Omega)$, we have that $-\Delta-B p(p+1)\left|v_{k}\right|^{p-1}$ possesses at least $k$ non-positive eigenvalues. Applying the following classical lemma to $V(x)=B p(p+1)\left|v_{k}(x)\right|^{p-1}$ and using the equation $\left\langle K^{\prime}\left(v_{k}\right), v_{k}\right\rangle=0=\left\|\nabla v_{k}\right\|_{2}^{2}-B(p+1)\left\|v_{k}\right\|_{p}^{p}$, we obtain the claimed energy estimate.

The following classical lemma can be found, for example, in Lieb]. 
Lemma 5.1. Let $V \in L^{n / 2}(\Omega)$ and denote by $m(V)$ the number of non-positive eigenvalues of the following eigenvalue problem:

$$
\left\{\begin{array}{rlr}
-\Delta u-V u=\lambda u & & \text { on } \Omega \\
u=0 & & \text { on } \partial \Omega .
\end{array}\right.
$$

(i) If $n \geq 3$, there is a constant $C_{n}>0$ such that $m(V) \leq C_{n}\|V\|_{n / 2}^{n / 2}$.

(ii) If $n=2$, then for any $\epsilon>0$ there is a constant $C_{\epsilon}>0$ such that $m(V) \leq$ $C_{\epsilon}\|V\|_{\epsilon+1}^{\epsilon+1}$ for all $V \in L^{\epsilon+1}(\Omega)$.

Remark 5.1. If $f$ is odd (i.e., $f(x,-s)=-f(x, s)$ ), the estimates in Proposition 5.2 combined with Proposition 2.1 yield the standard multiplicity result that problem (5.3) admits an unbounded sequence of solutions, provided $f$ satisfies the following superquadraticity condition à la Ambrosetti-Rabinowitz $[\mathrm{A}-\mathrm{R}]$ : for $q>2$ and some $R_{0}>0$

$$
0<q F(x, s) \leq f(x, s) s \text { for almost every } x \in \Omega \text { and }|s| \geq R_{0} .
$$

The superquadraticity is used to insure that almost critical sequences are bounded in $H_{0}^{1}$ and hence that $I$ satisfies the Palais-Smale condition. We shall come back to this topic in Section 7.

5.3. Morse indices, nodal sets and $L^{\infty}$-estimates. The Morse indices of the solutions of (5.3) - considered as critical points of the functional $I$ on $H_{0}^{1}(\Omega)$ - are not only relevant to the problems of existence and multiplicity of solutions but also to the study of the geometrical properties of these solutions as functions on the domain $\Omega$. For instance, they can be used to study the nodal set. Recall that, when $f(x, 0)=0$, the nodal set of a solution $u$ of $(5.3)$ is $u^{-1}(0)$. In the ODE case (Sturm-Liouville theory), the nodal set provides a complete understanding of the geometric structure of the solutions. In this case, there is a discrete set of eigenvalues and for each $k \geq 2$, the $k^{t h}$ eigenfunction has $k-1$ nodal points, which are all simple. However, Courant had shown some time ago that for PDEs, the number of nodal domains for solutions (i.e., the number of connected components of the non-zero set) may not be a good substitute for the number of nodal points in ODE's since he constructed examples where the $k^{\text {th }}$ eigenfunction has only two nodal domains for large $k$.

On the other hand, Coffman Co1 did generalize the Sturm-Liouville theory to certain super-linear eigenvalue ODE problems by relating the number of nodal points to the Morse indices of the solutions obtained variationally by means of the homological $\mathbf{Z}_{2}$-index. Later Bahri-Lions [Ba-L2] obtained estimates for superlinear elliptic equations with Dirichlet boundary conditions, finding that the number of nodal domains of a solution is always less than or equal to its Morse index and that, in the presence of symmetry, the $L^{\infty}$-norms of the min-max solutions go to $\infty$ with their Morse indices.

Another attempt to extend the ODE case came from Donnelly and Fefferman [D-F], who gave two-sided bounds for the Hausdorff measure of the nodal set for the $k^{\text {th }}$ eigenfunction of the Laplace-Beltrami operator on an $n$-dimensional analytic Riemannian manifold in terms of the $k^{\text {th }}$ eigenvalue:

$$
C_{1} \sqrt{k} \leq H^{n-1}\left(u_{k}^{-1}(0)\right) \leq C_{2} \sqrt{k},
$$

where $C_{1}, C_{2}>0$. 
In the following, we describe recent results of Xue-Fang Yang Ya where he establishes that indeed the $(n-1)$-dimensional Hausdorff measure of the nodal set may be a natural substitute for the number of nodal points in Sturm-Liouville theory when one deals with superlinear PDEs.

Example (9): Geometry of the solution set for superlinear elliptic problems. Consider again the boundary value problem (5.3) where the non-linearity $f$ and its antiderivative $F(x, u)=\int_{0}^{u} f(x, s) d s$ are $C^{1}(\Omega \times \mathbf{R})$ functions that satisfy the following hypotheses:

(H1) There exists $\mu>0$ such that $f_{u}(x, u) u^{2} \geq(1+\mu) f(x, u) u>0$ for large $|u|$ (Superlinearity).

(H2) There exists $0<\theta<1$ such that $\frac{2 n}{n-2} F(x, u) \geq(1+\theta) f(x, u) u$ for large $|u|$ (Subcritical growth).

(H3) There is a constant $C>0$ such that $|\nabla F(x, u)| \leq C(F(x, u)+1)$ for all $(x, u) \in \Omega \times \mathbf{R}$.

(H4) $f(x, 0)=0$ for all $x \in \Omega$.

If $\mathrm{f}$ is odd, we have just seen that problem (5.3) has then infinitely many solutions with both unbounded $L^{p}$-norms and Morse indices (see [R1], [St], G2] and their references). Bahri and Lions [Ba-L2] showed that if the non-linearity satisfies $f(x, t)|t|^{-p+1} t^{-1} \rightarrow c(x)>0$ uniformly on $\Omega$ as $t \rightarrow \infty$ where $c \in C(\bar{\Omega})$, then $m\left(u_{k}\right) \rightarrow \infty$ if and only if $\left\|u_{k}\right\|_{L^{\infty}} \rightarrow \infty$ where $u_{k}$ denotes the variationally obtained solutions. Their approach is an indirect method that uses a blow-up argument. More recently, Xue-Fang Yang [Ya] established the remarkable result that the $L^{\infty}$-norm of the solution is of at most polynomial growth in the Morse index. Then, using this result, a Carleman-type inequality, an iteration technique of Donelly-Fefferman [D-F] and some estimates of Hardt-Simon [H-S], he obtained exponential bounds on the vanishing order as well as on the Hausdorff measure of the nodal set.

To describe these results, recall that the vanishing order $\tau=\tau\left(u, x_{0}\right)$ of a nodal point $x_{0}$ of a solution $u$ is defined as the number $\tau$ such that for any $\epsilon>0$,

$$
\limsup _{x \rightarrow x_{0}}|u(x)|\left|x-x_{0}\right|^{-\tau+\epsilon}=0 \quad \text { and } \quad \limsup _{x \rightarrow x_{0}}|u(x)|\left|x-x_{0}\right|^{-\tau-\epsilon}=+\infty .
$$

Proposition 5.3. Assume $f$ satisfies (H1), (H2), and (H3). Then, if $u$ is a nonzero solution of (5.3) and if $m(u)$ is its Morse index as a critical point of the functional I defined in (5.2), then:

(i) There exist constants $C_{1}>0$ and $\beta>0$ depending on $\Omega, n, \mu, \theta$ and $\rho_{0}$, such that

$$
\|u\|_{L^{\infty}} \leq C_{1}(1+m(u))^{\beta} .
$$

If $f$ satisfies also $(\mathrm{H} 4)$, then

(ii) There exist $C_{2}=C_{2}\left(\Omega, n, \mu, \theta, \rho_{0}\right)>0$ and $\gamma=\gamma(\beta, \theta, n)>0$ such that for any nodal point $x_{0} \in \Omega$,

$$
\tau\left(u, x_{0}\right) \leq 2^{C_{2}(1+m(u))^{\gamma}} .
$$

(iii) There exists $C_{3}=C_{3}\left(\Omega, n, \mu, \theta, \rho_{0}\right)>0$ such that

$$
H^{n-1}\left(u^{-1}(0)\right) \leq 2^{2^{C_{3}(1+m(u))^{\gamma}}} \text {. }
$$




\section{Creating AND PReserving topological obstructions}

Now consider the problem

$$
\left\{\begin{array}{cl}
-\Delta u=|u|^{p-1} u+f & \text { on } \Omega \\
u=u_{0} & \text { on } \partial \Omega,
\end{array}\right.
$$

where $\Omega$ is a smooth, bounded, open subset of $R^{n}(n \geq 3), f \in C(\bar{\Omega}, R)$ and $u_{0} \epsilon C^{2}(\partial \Omega, R)$. In the case where $f=0$ and $u_{0}=0$, the problem is symmetric and its corresponding energy functional is even. As noted before, min-max principles for even functionals yield a multiplicity of solutions. In particular, in this case, there are infinitely many solutions with norms going to infinity for all subcritical values of $p$ (i.e., $1<p<\frac{n+2}{n-2}$ ).

If $u_{0}=0$ but $f$ is arbitrary, this symmetry is lost, and some partial success in dealing with this problem has been found in methods of "perturbation from symmetry". Bahri and Lions [Ba-L1] have the best result in this case, showing that (6.1) has an infinite number of solutions provided $1<p<\frac{n}{n-2}$.

When $u_{0}$ is non-trivial, the symmetry is again broken and the perturbationdue to the non-homogeneous boundary condition- is of higher order. The standard perturbative method can be applied but yields the result for an even smaller range of $p$ values. It was to deal with this type of perturbation that Bolle $\mathrm{Bo}$ developed his new approach (Theorem 6.1 below). Eventually, Bolle, Ghoussoub and Tehrani B-G-T tested this approach on several other problems, including (6.1), proving the existence of infinitely many solutions for a larger range, namely for $1<p<\frac{n+1}{n-1}$.

6.1. Preserving min-max levels after deformation of symmetry. We now present Bolle's Theorem: Consider two continuous functions $\rho_{1}, \rho_{2}:[0,1] \times \mathbf{R} \rightarrow \mathbf{R}$ with $\rho_{1} \leq \rho_{2}$, which are uniformly Lipschitz continuous with respect to the second variable. Associate to $\rho_{1}$ and $\rho_{2}$ the scalar flows $\psi_{1}, \psi_{2}:[0,1] \times \mathbf{R} \rightarrow \mathbf{R}$ defined by

$$
\left\{\begin{array}{l}
\psi_{i}(0, s)=s \\
\frac{\partial}{\partial \theta} \psi_{i}(\theta, s)=\rho_{i}\left(\theta, \psi_{i}(\theta, s)\right) .
\end{array}\right.
$$

They are continuous, increasing in $s$ and satisfy $\psi_{1} \leq \psi_{2}$.

Definition 6.1. Let $I_{0}$ be a $C^{2}$-functional satisfying $(P S)_{c}$ on a Hilbert space $E$. Say that a $C^{2}$-functional $I:[0,1] \times E \rightarrow \mathbf{R}$ is a good path of functionals starting at $I_{0}$ and controlled by $\rho_{1}, \rho_{2}$ if $I(0,)=.I_{0}$ and if it satisfies the following:

(I1) A Palais-Smale condition: Any sequence $\left(\theta_{n}, v_{n}\right)$ in $[0,1] \times E$ such that $I\left(\theta_{n}, v_{n}\right)$ is bounded and $\lim _{n \rightarrow \infty}\left\|I^{\prime}\left(\theta_{n}, v_{n}\right)\right\|=0$ has a convergent subsequence.

(I2) For each $b>0$, there exists a constant $C(b)$ depending on $b$ such that if $\left|I_{\theta}(v)\right| \leq b$, then $\left|\frac{\partial}{\partial \theta} I(\theta, v)\right| \leq C(b)\left(\left\|I_{\theta}^{\prime}(v)\right\|+1\right)(\|v\|+1)$.

(I3) For any critical point $v$ of $I_{\theta}$, we have $\rho_{1}\left(\theta, I_{\theta}(v)\right) \leq \frac{\partial}{\partial \theta} I(\theta, v) \leq \rho_{2}\left(\theta, I_{\theta}(v)\right)$.

(I4) For any finite dimensional subspace $W$ of $E$ and any $\theta \in[0,1]$, we have $\lim _{\|w\| \rightarrow \infty, w \in W} \sup _{\mu \in[0, \theta]} I(\mu, w)=-\infty$.

Here is the main idea behind Bolle's theorem: if one starts with a min-max critical level for the initial functional $I_{0}$, then only the deformation velocities $\rho_{1}$ and $\rho_{2}$ will determine whether this critical level persists along the path of functionals. We use the following notation: $\bar{\rho}_{i}(\theta, t)=\sup _{\mu \in[0, \theta]}\left|\rho_{i}(\mu, t)\right|$. 
- For subsets $U \subset V$ of $E$, set $c_{U}(\theta)=\sup _{U} I_{\theta}$ and $c_{V, U}(\theta)=\inf _{g \in S_{V, U}} \sup _{g(V)} I_{\theta}$ where

$$
S_{V, U}=\{g \in C(V, E) ; g(v)=v \text { on } U \text { and } g(v)=v \text { for }\|v\| \text { large }\} .
$$

- Say that a functional $I_{0}$ has a min-max configuration $(c, b)$ if there are two closed subsets $B \subset A \subset E$ such that $I_{0}$ is bounded on $A$ and $c:=c_{B, A}(0)>$ $b:=c_{B}(0)$.

If $c_{B, A}(0)>c_{B}(0)$, then, as seen before, $I_{0}$ has a critical point at level $c_{B, A}(0)$. Bolle's theorem states that, if $\psi_{1}\left(\theta, c_{B, A}(0)\right)>\psi_{2}\left(\theta, c_{B}(0)\right)$, this min-max critical level is preserved along any good path of functionals. More precisely, there are sets $A(\theta)$ and $B(\theta)$ in $E$ such that $c_{B(\theta), A(\theta)}(\theta)>c_{B(\theta)}(\theta)$, which means that again $I_{\theta}$ has a critical point at level $c_{B(\theta), A(\theta)}(\theta)$. This is the content of the following theorem.

Theorem 6.1. Let $I_{0}$ be a $C^{2}$-functional on $E$ with a min-max configuration $c_{B, A}(0)>C_{B}(0)$ associated to 2 closed subsets $B \subset A$ of $E$. Let $\rho_{1} \leq \rho_{2}$ be 2 uniformly Lipschitz velocity fields and let $\psi_{1}, \psi_{2}$ be the corresponding scalar flows. If $\psi_{1}\left(\theta_{0}, c_{B, A}(0)\right)>\psi_{2}\left(\theta_{0}, c_{B}(0)\right)$ for some $\theta_{0} \in[0,1]$, then for any path of functionals $I:[0,1] \times E \rightarrow \mathbf{R}$ satisfying (H1)-(H3) as well as

$$
\lim _{\substack{|v| \rightarrow \infty \\ v \in B}} \sup _{\theta \in\left[0, \theta_{0}\right]} I_{\theta}(v)=-\infty
$$

$I_{\theta_{0}}$ has a critical point at some level in the interval $\left[\psi_{1}\left(\theta_{0}, c_{B, A}(0)\right), \psi_{2}\left(\theta_{0}, c_{B, A}(0)\right)\right]$.

Here is an application as formulated by Chambers-Ghoussoub [C-G].

Theorem 6.2. Let $\rho_{1} \leq \rho_{2}$ be 2 velocity fields and let $\psi_{1}, \psi_{2}$ be the corresponding scalar flows. Assume the Hilbert space $E=E_{-} \oplus E_{+}$with $E_{-}$finite dimensional and let $\left(E_{k}\right)_{k=0}^{\infty}$ be subspaces of $E$ such that $E_{0}=E_{-}$and $E_{k}=E_{k-1} \oplus \mathbf{R} e_{k}$. Let $I_{0}$ be an even $C^{2}$-functional on $E$ and consider the levels $c_{k}=\inf _{h \in \mathcal{H}} \sup _{h\left(E_{k}\right)} I_{0}$, where

$$
\mathcal{H}=\{h \in C(E, E) ; h \text { is odd and } h(v)=v \text { for }\|v\| \text { large }\} .
$$

(i) If $\psi_{1}\left(\theta, c_{k}\right) \uparrow+\infty$ as $k \rightarrow \infty$, then for every integer $N$, there exists $\theta_{N} \in[0,1]$ -depending only on $I_{0}$ and $\rho_{1}, \rho_{2}$ - such that for any good path of functionals $I:[0,1] \times E \rightarrow \mathbf{R}$ starting at $I_{0}$ and controlled by $\rho_{1}, \rho_{2}$, the functional $I_{\theta}$ has at least $N$ distinct critical levels, for any $\theta \in\left[0, \theta_{N}\right]$.

(ii) If $c_{k} \geq B_{1}+B_{2} k^{\beta}$ where $\beta>0, B_{1} \in \mathbf{R}, B_{2}>0$ and $\bar{\rho}_{i}(\theta, t) \leq A_{1}+A_{2}|t|^{\alpha}$ where $0 \leq \alpha<1$ and $A_{1}, A_{2} \geq 0$, then $I_{1}$ has an unbounded sequence of critical levels provided $\beta>\frac{1}{1-\alpha}$.

Theorem 6.2 will follow from the following alternative: There is $C>0-$ depending only on $\rho_{1}$ and $\rho_{2}$ - such that for every $k \in \mathbf{N}$ and every $\theta \in[0,1]$ :

(1) either $\psi_{2}\left(\theta, c_{k}\right)<\psi_{1}\left(\theta, c_{k+1}\right)$,

(2) or $c_{k+1}-c_{k} \leq C \theta\left(\overline{\rho_{1}}\left(\theta, c_{k+1}\right)+\overline{\rho_{2}}\left(\theta, c_{k}\right)+1\right)$.

Moreover, in case (1) there exists a level $\ell_{k}(\theta)$ - only depending on $I_{0}$ and $\rho_{1}, \rho_{2}-$ such that for any good path of functionals $I:[0,1] \times E \rightarrow \mathbf{R}$ starting at $I_{0}$, controlled by $\rho_{1}, \rho_{2}$, there exists a critical level $\overline{c_{k}}(\theta)$ for $I_{\theta}$ with $\psi_{1}\left(\theta, c_{k+1}\right) \leq \overline{c_{k}}(\theta) \leq \ell_{k}(\theta)$.

If we assume that $\psi_{2}\left(\theta, c_{k}\right) \geq \psi_{1}\left(\theta, c_{k+1}\right)$, then alternative (2) follows from the fact that $\rho_{i}$ is uniformly Lipschitz in the second variable. 
Now suppose that $\psi_{2}\left(\theta, c_{k}\right)<\psi_{1}\left(\theta, c_{k+1}\right)$. Let $\varepsilon>0$ be such that $\psi_{2}\left(\theta, c_{k}+\varepsilon\right)<$ $\psi_{1}\left(\theta, c_{k+1}\right)$ and choose $h \in \mathcal{H}$ such that $\sup _{h\left(E_{k}\right)} I_{0}<c_{k}+\varepsilon$. Let $E_{k+1}^{+}=E_{k} \oplus$ $\mathbf{R}^{+} e_{k+1}$ and set $A_{k}=h\left(E_{k+1}^{+}\right)$and $B_{k}=h\left(E_{k}\right)$. We claim that the hypotheses of Bolle's theorem are satisfied for such sets.

Indeed, to establish that $\psi_{1}\left(\theta, c_{B_{k}, A_{k}}(0)\right)>\psi_{2}\left(\theta, c_{B_{k}}(0)\right)$, consider any $g \in$ $S_{B_{k}, A_{k}}$ and note that $p=\left.g \circ h\right|_{E_{k+1}^{+}}$is odd on $E_{k}$ since $h$ is odd and $g$ is the identity on $B_{k}=h\left(E_{k}\right)$. Since $E_{k+1}^{+}=E_{k} \oplus \mathbf{R}^{+} e_{k+1}$, use the Tietze extension theorem to extend $p$ to an odd function on $E_{k+1}$ and consequently to a function $\bar{p}$ in $\mathcal{H}_{k}$. We now find, since $I_{0}$ is even:

$$
\sup _{g\left(A_{k}\right)} I_{0}=\sup _{p\left(E_{k+1}^{+}\right)} I_{0}=\sup _{\bar{p}\left(E_{k+1}\right)} I_{0} \geq \inf _{h \in \mathcal{H}} \sup _{h\left(E_{k+1}\right)} I_{0}=c_{k+1}
$$

and hence $c_{B_{k}, A_{k}}(0)=\inf _{g \in S_{B_{k}, A_{k}}} \sup _{g\left(A_{k}\right)} I_{0} \geq c_{k+1}$. Note also that

$$
c_{B_{k}}(0)=\sup _{B_{k}} I_{0}=\sup _{h\left(E_{k}\right)} I_{0}<c_{k}+\varepsilon<c_{k+1} .
$$

These two estimates imply $c_{B_{k}, A_{k}}(0)>c_{B_{k}}(0)$ and

$$
\psi_{1}\left(\theta, c_{B_{k}, A_{k}}(0)\right) \geq \psi_{1}\left(\theta, c_{k+1}\right)>\psi_{2}\left(\theta, c_{k}+\varepsilon\right) \geq \psi_{2}\left(\theta, c_{B_{k}, A_{k}}(0)\right) .
$$

So we can indeed apply Bolle's theorem and conclude that $I_{\theta}$ has a critical level $\overline{c_{k}}(\theta)$ in the interval $\left[\psi_{1}\left(\theta, c_{B_{k}}, A_{k}(0)\right), \psi_{2}\left(\theta, c_{B_{k}, A_{k}}(0)\right)\right]$. Thus, as announced:

$$
\ell_{k}(\theta):=\psi_{2}\left(\theta, c_{B_{k}, A_{k}}(0)\right) \geq \overline{c_{k}}(\theta) \geq \psi_{1}\left(\theta, c_{B_{k}, A_{k}}(0)\right) \geq \psi_{1}\left(\theta, c_{k+1}\right)>\psi_{2}\left(\theta, c_{k}\right) .
$$

To prove (i) of Theorem 6.2 , define for every $k$ the necessarily positive values:

$$
\eta_{k}=\inf \left\{\theta ; c_{k+1}-c_{k} \leq C \theta\left(\overline{\rho_{1}}\left(\theta, c_{k+1}\right)+\overline{\rho_{2}}\left(\theta, c_{k}\right)+1\right)\right\},
$$

and use the fact that the sequences $c_{k}$ and $\psi_{1}\left(\theta, c_{k}\right)$ are unbounded.

For (ii) first note that $\psi_{1}(1, s) \geq s-\overline{\rho_{1}}(1, s) \geq s-A_{1}-A_{2}|s|^{\alpha}$. Since $c_{k}$ is unbounded, so is $\psi_{1}\left(1, c_{k}\right)$. If now $I_{1}$ has only finitely many solutions, then alternative (2) above must hold for large $k$; hence

$$
c_{k+1}-c_{k} \leq C\left(\overline{\rho_{1}}\left(1, c_{k+1}\right)+\overline{\rho_{2}}\left(1, c_{k}\right)\right) \leq C\left(A_{1}+A_{2}\left|c_{k+1}\right|^{\alpha}+B_{1}+B_{2}\left|c_{k}\right|^{\alpha}\right) .
$$

This implies $c_{k} \leq C_{1}+C_{2} k^{\frac{1}{1-\alpha}}$ for some $C_{1} \in \mathbf{R}$ and $C_{2}>0$. However, by assumption, $c_{k} \geq B_{1}+B_{2} k^{\beta}$. This is a contradiction whenever $\beta>\frac{1}{1-\alpha}$.

\subsection{Multiplicity results for non-homogeneous equations.}

Example (10): Superlinear equations with a non-homogeneous perturbative term. We now apply Theorem 6.2 to obtain multiple solutions for semi-linear elliptic partial differential equations of the form:

$$
\left\{\begin{aligned}
-\Delta u & =p(x, u)+f(\theta, x, u) & & \text { on } \Omega \\
u & =0 & & \text { on } \partial \Omega,
\end{aligned}\right.
$$

where $p(x, \cdot)$ is an odd non-linearity and $f$ is a perturbative term. Again, define $P$ and $F$ as $P(x, \zeta)=\int_{0}^{\zeta} p(x, z) d z$ and $F(\theta, x, \zeta)=\int_{0}^{\zeta} f(\theta, x, z) d z$ respectively and make the following standard superlinearity assumptions on the symmetric term $p$.

(A) (i) $p \in C(\bar{\Omega} \times \mathbf{R}, \mathbf{R})$ and $p(x, \cdot)$ is odd.

(ii) $|p(x, \zeta)| \leq a_{1}+a_{2}|\zeta|^{s}$ for some $a_{1}, a_{2}>0$ and $0 \leq s<\frac{n+2}{n-2}$.

(iii) There is $r>2$ such that $0 \leq r P(\theta, x, \zeta) \leq \zeta p(\theta, x, \zeta)$ for $|\zeta|$ large. 
Here are our assumptions on the perturbative term $f$ :

(B) (i) $f \in C([0,1] \times \bar{\Omega} \times \mathbf{R}, \mathbf{R})$ and $f(0, \cdot, \cdot)=0$.

(ii) There are $1 \leq s_{1}<r$ and $c_{1}, c_{2}>0$ such that $\left|\frac{\partial}{\partial \theta} F(\theta, x, \zeta)\right| \leq$ $c_{1}|\zeta|^{s_{1}}+c_{2}$.

(iii) There is $b>0$ and $g \in L_{+}^{2^{*}-1}(\Omega)$ such that $f(\theta, x, \zeta) \leq$ $b|\xi|^{s_{1}-1}+g(x)$ for $|\zeta|$ large.

(iv) There are $0<s_{2}<r$ and $d_{1}, d_{2}>0$ such that $\left|\int_{\Omega} \frac{\partial}{\partial \theta} F(\theta, x, u) d x\right| \leq d_{1}\|u\|_{r}^{s_{2}}+d_{2}$ whenever $u$ is a solution of $(6.2)$.

The following is a recent result of Chambers-Ghoussoub $\mathrm{C}-\mathrm{G}$.

Theorem 6.3. (i) Suppose $p$ satisfies (A). Then, for every $N$, there exists $\theta_{N} \in$ $(0,1]$ such that $(6.2)$ has at least $N$ solutions for each $\theta \in\left[0, \theta_{N}\right]$ and any $f$ satisfying (B).

(ii) Suppose (A) and (B) are satisfied. If in addition $\frac{s+1}{s-1} \frac{2}{n}>\frac{r}{r-s_{2}}$, then problem (6.2) has an infinite number of solutions for all $\theta \in[0,1]$.

Clearly (B)(ii) implies (B)(iv) with $s_{2}=s_{1}$. However, as Theorem 6.3 indicates, the smaller $s_{2}$ is, the sharper the result obtained. The fact that (B)(iv) is only required for a-priori solutions of (6.2) allows us to go for lower exponents $s_{2}$ and hence for higher non-linearities $s$.

To sketch a proof, consider the path of functionals $I:[0,1] \times E \rightarrow \mathbf{R}$ by

$$
I(\theta, u)=I_{\theta}(u)=\int_{\Omega}\left(\frac{1}{2}|\nabla u|^{2}-P(x, u)-F(\theta, x, u)\right) d x
$$

on the Hilbert space $E=H_{0}^{1}(\Omega)$. It is clear that $I_{0}$ is an even functional and that the solutions of (6.2) are the critical points of $I_{\theta}$. It is relatively standard to check that $I$ satisfies (I1), (I2) and (I4). Now let $E_{k}$ be the subspace of $E$ spanned by the first $k$ eigenfunctions of $\Delta$. Let

$$
\mathcal{G}=\{g \in C(E, E) ; g \text { is odd and } g(x)=x \text { for large }\|x\|\} .
$$

Set $c_{k}=\inf _{g \in G} \sup _{g\left(E_{k}\right)} I_{0}$. From Section 5.2, we know that these $c_{k}$ 's are critical levels of $I_{0}$ and that $c_{k} \geq B_{1} k^{\frac{s+1}{s-1} \frac{2}{n}}-B_{2}$ for some $B_{1}, B_{2}>0$. Finally, one shows that (I3) holds with

$$
\rho_{1}(\theta, t)=-C\left(t^{2}+1\right)^{\frac{s_{2}}{2 r}} \text { and } \rho_{2}(\theta, t)=C\left(t^{2}+1\right)^{\frac{s_{2}}{2 r}} .
$$

So we can apply Theorem 6.2(ii) with $\alpha=\frac{s_{2}}{r}$ and $\beta=\frac{s+1}{s-1} \frac{2}{n}$ to obtain that $I_{1}$ has an infinite number of solutions when $\frac{s+1}{s-1} \frac{2}{n}=\beta \geq \frac{1}{1-\alpha}=\frac{r}{r-s_{2}}$, which is the claim in Theorem 6.3(ii). Theorem 6.3(i) also follows directly from Theorem 6.3(i), since $\psi_{1}\left(\theta, c_{k}\right) \uparrow+\infty$ as $k \rightarrow \infty$ for the $\rho_{i}$ defined above. We now apply this result to two classical examples.

Example (11): Superlinear equations with non-homogeneous Dirichlet boundary conditions. Consider the following boundary value problem:

$$
\left\{\begin{aligned}
-\Delta u & =|u|^{p-1} u+f & & \text { on } \Omega \\
u & =u_{0} & & \text { on } \partial \Omega,
\end{aligned}\right.
$$

and associate the following parametrized version for $\theta \in[0,1]$ :

$$
\left\{\begin{aligned}
-\Delta u & =|u|^{p-1} u+\theta f & & \text { on } \Omega \\
u & =\theta u_{0} & & \text { on } \partial \Omega .
\end{aligned}\right.
$$


Proposition 6.1. With the above notation:

(i) If $1<p<\frac{n+2}{n-2}$, then for any integer $N$, there exist $\theta_{N}>0$ such that for any $f$ with $\|f\| \frac{p+1}{p} \leq \theta_{N}$ and any $u_{0}$ with $\left\|u_{0}\right\|_{p+1} \leq \theta_{N}$, problem (6.3) has at least $N$ solutions.

(ii) If $u_{0}=0$ and if $1<p<\frac{n}{n-2}$, then (6.3) has an infinite number of solutions for any $f$ in $H^{-1}(\Omega)$.

(iii) If $1<p<\frac{n+1}{n-1}$, then for any $f \in H^{-1}(\Omega)$ and any $u_{0} \in C(\partial \Omega)$, problem (6.3) has infinitely many solutions.

If $u_{0}=0$, then by applying Theorem 6.3 with $s=p, r=p+1, s_{1}=s_{2}=1$, one recovers the result in [Ba-L1] stating that there is an infinite number of solutions provided $p$ is below $\frac{n}{n-2}$.

If $u_{0}$ is not zero, use the change of variables $v=u-\theta u_{0}$ where $u_{0}$ denotes the harmonic extension of its values on $\partial \Omega$, and rewrite equation (6.4) in terms of $v$ :

$$
\left\{\begin{array}{cl}
-\Delta v=\left|v+\theta u_{0}\right|^{p-1}\left(v+\theta u_{0}\right)+\theta f & \text { on } \Omega \\
v=0 & \text { on } \partial \Omega .
\end{array}\right.
$$

To establish (i) and (iii), one applies Theorem 6.3 with $p(x, v)=|v|^{p-1} v$ and

$$
f(\theta, x, u)=\left|v+\theta u_{0}\right|^{p-1}\left(v+\theta u_{0}\right)-|v|^{p-1} v+\theta f .
$$

Hypotheses (A) and (B) (i-iii) are easily checked, with $s=p, r=p+1$ and $s_{1}=p$. That (B)(iv) is satisfied with $s_{2}=\frac{p+1}{2}$ is more involved and requires a Pohozaev type argument (see $[\mathrm{B}-\mathrm{G}-\mathrm{T}]$ ). One then obtains that $I_{1}$ has an infinite number of solutions provided $p<\frac{n+1}{n-1}$. Whether the above results still hold for $p$ all the way up to the critical Sobolev exponent $2^{*}-1=\frac{n+2}{n-2}$ remains open.

6.3. Tensor products of min-max classes. E. Séré introduced in $\mathrm{Se}$ a powerful and novel variational approach to deal with the multiplicity of homoclinic orbits for a family of first order periodic Hamiltonian systems. This method was later developed by Coti-Zelati and Rabinowitz [C-R1], [C-R2] and others to obtain multibump solutions for elliptic partial differential equations.

The basic idea in Séré's method is as follows: given one low energy solution, translate its support in different directions and far apart until their interference with each other is sufficiently weakened, then patch the pieces together to create "multi-bump" solutions, i.e., solutions with most of their mass lying in a finite disjoint union of balls.

From a variational point of view, the least-energy solutions are usually obtained by the mountain pass procedure. That is, assuming 0 is a local minimum for the functional $I$ in question, the least non-trivial critical level is usually given by the mountain pass value $c$. Since the expected energy of $k$ glued and non-mutually interfering solutions is supposed to be $k c$, then one may try the idea of "adding up" mountain pass classes in an appropriate fashion.

One way to do it, assuming $E$ is now a function space, is to consider a finite family of disjoint open sets $\left\{\Omega_{i} ; i=1, \ldots, k\right\}$ and to define a "tensor product" of mountain pass families as follows: For $\theta=\left(\theta_{1}, \ldots, \theta_{k}\right) \in[0,1]^{k}$, let $0_{i}=$ $\left(\theta_{1}, \ldots, \theta_{i-1}, 0, \theta_{i+1}, \ldots, \theta_{k}\right)$ and $1_{i}=\left(\theta_{1}, \ldots, \theta_{i-1}, 1, \theta_{i+1}, \ldots, \theta_{k}\right)$ where $i=1,2, \ldots, k$. For any function $u \in E$, use $\operatorname{supp}(u)$ to denote its support in $\mathbf{R}^{n}$. Set

$$
\Gamma_{k}=\left\{\mathcal{H}=h_{1}+h_{2}+\cdots+h_{k} ; h_{i} \text { satisfies }(H 1)-(H 3), i=1,2, \ldots, k\right\}
$$

where 
(H1) $h_{i} \in C\left([0,1]^{k}, E\right)$;

(H2) $h_{i}\left(0_{i}\right)=0$ and $I\left(h_{i}\left(1_{i}\right)\right)<0$;

(H3) $\operatorname{supp} h_{i}(\theta) \subset \Omega_{i}$ for all $\theta \in[0,1]^{k}$.

Call an element in $\Gamma_{k}$ a " $k$-path" and let $b=\inf _{\mathcal{H} \in \Gamma_{k}} \sup _{\theta \in[0,1]^{k}} I(\mathcal{H}(\theta))$.

Such a class is not homotopy stable, and therefore one cannot conclude that $b$ is a new critical level. However, it turns out that in many cases, one can approximate a $k$-path deformed along the negative gradient flow with another $k$-path without changing the energy level by much and the usual deformation lemmas apply to get critical levels.

Even though this procedure has been applied in many situations, no general principle has yet been developed and each application seems to need its own adhoc argumentation. We now describe Gui's adaptation of this method to obtain multi-peak solutions for Neumann problems Gu1, Gu2].

Example (12): A positive solution to a Neumann problem involving the critical exponent. We are here interested in the existence and in the qualitative behavior as $\lambda \rightarrow+\infty$ of solutions to the following problem:

$$
\left\{\begin{aligned}
\Delta u-\lambda u+u^{p}=0 & \text { on } \Omega \\
u>0 & \text { on } \Omega \\
\frac{\partial u}{\partial \nu}=0 & \text { on } \partial \Omega,
\end{aligned}\right.
$$

where $\Omega \subset \mathbf{R}^{n}$ is a bounded smooth domain, $n \geq 3, \lambda \geq 0$, and $p=\frac{n+2}{n-2}$. The corresponding energy functional is

$$
I_{\lambda}(u)=\frac{1}{2} \int_{\Omega}|\nabla u|^{2}+\frac{\lambda}{2} \int_{\Omega}|u|^{2}-\frac{1}{p+1} \int_{\Omega}|u|^{p+1}
$$

which is $C^{2}$ on the space $E_{\lambda}=\left\{u \in H^{1}(\Omega) ;\|u\|_{\lambda}^{2}:=\int_{\Omega}\left(|\nabla u|^{2}+\lambda|u|^{2}\right) d x<+\infty\right\}$.

To find non-trivial solutions, one has to deal with the lack of compactness in the Sobolev embedding. Using a variational approach in the same spirit as $[\mathrm{B}-\mathrm{N}]$ for the Dirichlet problem (see also Section 2), Adimurthi, Comte, Mancini, Yadava, Pacella, Ni, Pan, Takagi, Wang, and others obtained results in this direction. For details see the references in $\mathrm{G}-\mathrm{G}$. Let

$$
S:=\inf _{\substack{\varphi \in H^{1}\left(\mathbf{R}^{n}\right) \\ \varphi \neq 0}} \frac{\|\nabla \varphi\|_{L^{2}\left(\mathbf{R}^{n}\right)}^{2}}{\|\varphi\|_{L^{p}\left(\mathbf{R}^{n}\right)}^{2}}=n(n-2) \pi\left[\frac{\Gamma(n / 2)}{\Gamma(n)}\right]^{\frac{2}{n}}
$$

be the best constant in the Sobolev embedding. In order to use the mountain pass procedure, define again $\mathcal{F}_{1}=\left\{h \in C^{1}\left([0,1]: E_{\lambda}\right) ; h(0)=0, I_{\lambda}(h(1))<0\right\}$ which is dual to the Nehari manifold $M_{1}=\left\{u \in E_{\lambda}: u \neq 0\right.$ and $\left.\left\langle I_{\lambda}^{\prime}(u), u\right\rangle=0\right\}$.

Theorem 6.4. Suppose $n \geq 3$ and $\lambda>0$. Then the following hold:

(i) $c_{\lambda}:=c\left(I_{\lambda}, \mathcal{F}_{1}\right)=\inf _{M_{1}} I_{\lambda}<\frac{1}{2 n} S^{n / 2}$.

(ii) $I_{\lambda}$ satisfies $(P S)_{c}$ for any $c<\frac{1}{2 n} S^{n / 2}$, and consequently, (6.5) has a non-zero least energy solution $u_{\lambda}$ at energy level $c_{\lambda}$.

(iii) If $n \geq 5$, then for $\lambda$ large enough, $u_{\lambda}$ has a unique maximum point $P_{\lambda}$ near the points of $\partial \Omega$ where the mean curvature $H$ attains its maximum.

Actually, if one sets $U(x)=\left(\frac{n(n-2)}{n(n-2)+|x|^{2}}\right)^{\frac{n-2}{2}}$ and $U_{\epsilon, y}(x)=\epsilon^{-\frac{n-2}{2}} U\left(\frac{x-y}{\epsilon}\right)$ for $\epsilon>0$ and $y \in \mathbf{R}^{n}$, then the following hold: 
- $\lim _{\lambda \rightarrow \infty}\left\|\left(u_{\lambda}-U_{\epsilon_{\lambda}, P_{\lambda}}\right)\right\|_{L^{2}}=0$ where $\epsilon_{\lambda}^{(n-2) / 2}=1 / u_{\lambda}\left(P_{\lambda}\right)$.

- The limit points of $\left(P_{\lambda}\right)_{\lambda}$ as $\lambda \rightarrow \infty$ are contained in the set of points of maximum mean curvature of $\partial \Omega$.

One estimates the maximum (in $t$ ) of $I_{\lambda}\left(t U_{\epsilon, y}\right)$, where $y \in \partial \Omega$ and $\epsilon \rightarrow 0, \lambda \rightarrow+\infty$ in such a way that $\frac{1}{\epsilon \sqrt{\lambda}} \rightarrow \infty$. Careful computations lead to

$$
I_{\lambda}\left(t U_{\epsilon, y}\right) \leq \frac{1}{2 n} S^{n / 2}-p_{n} H(y)^{2} \frac{1}{\lambda}+o\left(\frac{1}{\lambda}\right),
$$

where $p_{n}$ is a positive constant and $H$ is the curvature at $y$ and from that to the crucial estimate

$$
c_{\lambda}=\frac{1}{2 n} S^{n / 2}-p_{n} M^{2} \frac{1}{\lambda}+o\left(\frac{1}{\lambda}\right),
$$

where $M$ is the maximum of the mean curvature of $\partial \Omega$. By localizing the argument, one can actually show that there are one-peak solutions which concentrate near any local maximum point of the boundary's mean curvature.

6.4. Multi-peak solutions for singularly perturbed problems. More recently, the problem of finding multi-peak solutions that concentrate near several local maxima of the mean curvature was tackled by Gui Gu1, Gu2 in the subcritical case and by Ghoussoub and Gui [G-G] in the critical case. Now, from the asymptotic analysis, one can see these one-peak solutions concentrating around some $P_{i}$, being exponentially small in $-\sqrt{\lambda}$ in the region near the other $P_{j}$ 's. Therefore, intuitively these solutions interact very little with each other, and there should be a solution to (6.5) which is close to the superposition of these one-peak solutions when $\lambda$ is sufficiently large. This could have been done via the implicit function theorem if the required spectrum properties of the linearized equation could be obtained. However, these conditions are not satisfied here.

On the other hand, it is clear that in any attempt to obtain such solutions variationally, one needs to carefully choose the procedure in order to get the appropriate critical level (roughly the sum of the levels of the basic localized one-peak solutions) and to force the critical point to be close to the basic multi-peak functions $\Sigma_{i} c_{i} U_{\epsilon_{i}, y_{i}}$. Again, additional care needs to be taken to deal with the lack of compactness.

The variational method pioneered by Séré discussed above does provide the right tool for "gluing" one-peak solutions. The main idea is now to min-max the functional over a "tensor product" of "mountain passes" which may eventually catch the information on the local "mountain pass-type" structure of the functions that concentrate near $P_{i}$ for each $i=1,2, \ldots, k$, and therefore to obtain a multi-peak solution.

Example (13): Multi-peak solutions for a singularly perturbed Neumann problem involving the critical exponent. Assume the following condition on the smooth domain $\Omega$ :

- There exist $k$ disjoint smooth patches $\Lambda_{i}, 1 \leq i \leq k$ in $\partial \Omega$, each one being the closure of a connected open set with smooth boundary such that $H(y) \geq 0$ for $y \in \Lambda_{i}$ and

$$
M_{i}:=\max _{y \in \Lambda_{i}} H(y)>m_{i}:=\max _{y \in \partial \Lambda_{i}} H(y), \quad i=1,2, \ldots, k .
$$

Here is the result of Gui-Ghoussoub [G-G]. 
Theorem 6.5. Suppose that $n \geq 5$ and that (6.7) holds. Then, for $\lambda$ sufficiently large, problem (6.5) has a classical positive solution $u_{\lambda}$ that possesses exactly $k$ local maximum points $P_{\lambda, i}, i=1,2, \ldots, k$ with the following properties:

(i) Each $P_{\lambda, i}$ belongs to $\Lambda_{i}$;

(ii) $\lim _{\lambda \rightarrow \infty} H\left(P_{\lambda, i}\right)=M_{i}$;

(iii) $\lim _{\lambda \rightarrow \infty} u_{\lambda}\left(P_{\lambda, i}\right)=\infty$.

(iv) For any $\epsilon>0$, there exist positive constants $\lambda_{0}$ and $R$ such that for $\lambda \geq \lambda_{0}$ and $x$ in $\Omega \backslash \cup_{i=1}^{k} B_{R / \sqrt{\lambda}}\left(P_{\lambda, i}\right)$, we have $0<u_{\lambda}(x) \leq C \epsilon \exp (-\sigma d(x) \sqrt{\lambda})$, where $C>0, \sigma>0$ are positive constants only dependent on $\Omega$, and $d(x)$ is the distance function to the peaks, i.e. $d(x)=\min \left\{\left|x-P_{\lambda, i}\right|, i=1,2, \ldots, k\right\}$.

We sketch the main steps of the proof. First, construct $k$ subdomains $\Omega_{i}, i=$ $1,2, \ldots, k$ of $\Omega$ such that $\bar{\Omega}_{i} \cap \bar{\Omega}_{j}=\emptyset, \forall i \neq j, \partial \Omega_{i} \cap \partial \Omega=\Lambda_{i}$, and $\partial \Omega_{i}^{+}:=\partial \Omega_{i} \backslash \partial \Omega$ is smooth and orthogonal to $\partial \Omega$ at $\partial \Lambda_{i}$ for $i=1,2, \ldots, k$. Then, localize the equation to each patch in such a way that the mountain pass solution of the corresponding functional peaks in that patch. For that, define for $i=1,2, \ldots, k$,

$$
g_{i}(x, u)= \begin{cases}|u|^{p-1} u & \text { if } x \in \Omega_{i} \text { or }|u| \leq 1 \\ u & \text { if } x \in \Omega \backslash \Omega_{i} \text { and }|u| \geq 1\end{cases}
$$

and

$$
I_{\lambda, i}(u)=\int_{\Omega}\left(\frac{1}{2}\left(|\nabla u|^{2}+\lambda u^{2}\right)-G_{i}(x, u)\right) d x, \quad u \in E_{\lambda}
$$

where $G_{i}(x, u)$ is the primitive of $g_{i}(x, u)$. As above, if we define

$$
\mu_{\lambda, i}=\inf _{h \in \mathcal{F}_{1}} \sup _{t \in[0,1]} I_{\lambda, i}(h(t))=\inf _{\substack{u \neq 0 \\ u \in E_{\lambda}}} \sup _{t>0} I_{\lambda, i}(t u),
$$

then

$$
\mu_{\lambda, i}=\frac{1}{2 n} S^{n / 2}-p_{n} M_{i}^{2} \frac{1}{\lambda}+o\left(\frac{1}{\lambda}\right)
$$

for some $p_{n}>0$, and one obtains mountain pass points for $I_{\lambda, i}$ corresponding to one-peak solutions for each $i=1,2, \ldots, k$ that do concentrate for large $\lambda$.

To obtain multi-peak solutions, use the tensor products of mountain passes described above. Consider the levels

$$
b_{\lambda}=\inf _{\mathcal{H} \in \Gamma_{\lambda}} \sup _{\theta \in[0,1]^{k}} I_{\lambda}(\mathcal{H}(\theta)) \quad \text { and } \quad \mu_{\lambda}=k \frac{1}{2 n} S^{n / 2}-p_{n}\left(\sum_{i=1}^{k} M_{i}^{2}\right) \frac{1}{\lambda}
$$

where $\Gamma_{\lambda}=\Gamma_{\lambda, k}$ is the set of $k$-paths corresponding to $I_{\lambda}$ defined above. As in the case of one-peak solutions, the initial step is to show that $b_{\lambda}=\mu_{\lambda}+o\left(\frac{1}{\lambda}\right)$ for large $\lambda$. For that, note that if each $h_{i}$ satisfies (H1)-(H3), a standard topological argument yields the existence of a $\bar{\theta} \in[0,1]^{k}$ such that $I_{\lambda}\left(h_{i}(\bar{\theta})\right) \geq \mu_{\lambda, i}$ for all $i$. Since $I_{\lambda}(u)=I_{\lambda, i}(u)$ when $\operatorname{supp}(u) \subset \Omega_{i}$, this combined with (6.6) yields that $b_{\lambda} \geq \mu_{\lambda}+o\left(\frac{1}{\lambda}\right)$. For the reverse inequality, we need to construct a $\mathcal{H}_{\lambda}(\theta) \in \Gamma_{\lambda}$ with

$$
\sup _{\theta \in[0,1]^{k}} I_{\lambda}\left(\mathcal{H}_{\lambda}(\theta)\right) \leq \mu_{\lambda}+o\left(\frac{1}{\lambda}\right) \text { as } \lambda \rightarrow \infty .
$$

For that, choose $y_{i} \in \Lambda_{i}$ with $H\left(y_{i}\right)=M_{i}$ and hence satisfying $d\left(y_{i}, \Omega \backslash \Omega_{i}\right)>0$ for $i=1,2, \ldots, k$. Pick a smooth cut-off function $\chi(x)$ such that $\chi(x)=1$ when $|x| \leq 1$ 
and $\chi(x)=0$ when $|x| \geq 2$. Let $\chi_{\rho}(x)=\chi(x / \rho)$ for $\rho>0$. For any $\epsilon>0, y \in R^{n}$, let $\rho=\frac{1}{\epsilon \sqrt{\lambda}}$, and define

$$
U_{\epsilon, y}=\epsilon^{-\frac{n-2}{2}} U\left(\frac{x-y}{\epsilon}\right), \quad U_{\epsilon, y, \lambda}=\epsilon^{-\frac{n-2}{2}}\left(\chi_{\rho} U\right)\left(\frac{x-y}{\epsilon}\right) \quad \text { and } u_{i}=U_{\bar{\epsilon}, y_{i}, \lambda},
$$

where $\bar{\epsilon}$ is appropriately chosen. It is easy to see that there exists a constant $T>0$ such that $I_{\lambda}\left(T u_{i}\right)<0$ for $i=1,2, \ldots, k$. Now define $h_{i}(\theta)=\theta_{i} T u_{i}$ for $\theta \in[0,1]^{k}$ and $\mathcal{H}_{\lambda}(\theta)=h_{1}(\theta)+h_{2}(\theta)+\cdots+h_{k}(\theta)$. When $\lambda$ is sufficiently large, $\mathcal{H}_{\lambda} \in \Gamma_{\lambda}$ and $I_{\lambda}\left(\mathcal{H}_{\lambda}(\theta)\right)=\sum_{i=1}^{k} I_{\lambda}\left(h_{i}(\theta)\right)$. Therefore

$$
\sup _{\theta \in[0,1]^{k}} I_{\lambda}\left(\mathcal{H}_{\lambda}(\theta)\right) \leq \mu_{\lambda}+o\left(\frac{1}{\lambda}\right) \quad \text { as } \quad \lambda \rightarrow \infty .
$$

Unlike the case of a one-peak solution, there are still many problems to overcome:

(P1) The class $\Gamma_{\lambda}$ is not homotopy stable and therefore it is not at all clear that $b_{\lambda}$ is a critical level. This problem can be overcome as one can approximate the $k$-path deformed along the negative gradient flow by another $k$-path. This is due to the weak interference between peaks that concentrate in different regions.

(P2) Unlike the case $k=1$, the fact that $b_{\lambda}=\mu_{\lambda}+o\left(\frac{1}{\lambda}\right)$ will not suffice to show that the critical point obtained is a $k$-peak solution. For that, one needs to locate critical points that are close - in an appropriate metric - to the set

$$
\mathcal{M}=\left\{v ; v=\sum_{i=1}^{k} U_{\epsilon_{i}, y_{i}} ; \epsilon_{i}>0 \quad \text { and } \quad y_{i} \in \Lambda_{i} \text { for all } i=1, \ldots, k\right\}
$$

of "elementary" multi-peak functions.

(P3) There is also a lack of compactness due to the critical exponent. But this could be overcome by proving the Palais-compactness "around the set" $\mathcal{M}$.

Again, we refer to Gu1], Gu2 and [G-G] for more details and references.

\section{Parameter Dependent Functionals AND COMPACTNESs}

In certain variational problems which depend on some parameter $\lambda \in T \subset \mathbf{R}^{+}$, one can associate energy levels $c_{\lambda}$ of the form $c\left(I_{\lambda}, \mathcal{F}_{\lambda}\right)$ to some functionals $I_{\lambda}$ and homotopy stable families $\mathcal{F}_{\lambda}$. If the function $\lambda \rightarrow c_{\lambda}$ happens to have a monotonicity property, then the fact that it is differentiable on some dense subset $S$ of $T$ can sometimes lead to better bound estimates on the approximate critical sequences and therefore to a weakening of the required Palais-Smale condition. This trick introduced by Struwe in the context of surfaces of prescribed mean curvature St1. - has found many applications: in the context of Ginzburg-Landau functionals St], in Chern-Simon theory [St-Ta and in mean field theory D-J-L-W. Recently, Jeanjean $[J]$ formulated an abstract result of this type in the mountain pass setting where only the functionals are parameter dependent. Here is a description in a more general situation.

\subsection{Constructing bounded Palais-Smale sequences.}

Theorem 7.1. Let $\left(I_{\lambda}\right)_{\lambda \in T}$ be a family of $C^{1}$-functionals on a Banach space $X$, of the form $I_{\lambda}(x)=J_{1}(x)-\lambda J_{2}(x)$ where $J_{2} \geq 0$ and $J_{2}(x) \rightarrow+\infty$ when $\|x\| \rightarrow+\infty$. Suppose $\mathcal{F}$ is a homotopy stable family with boundary $B$ such that for each $\lambda$, $\sup I_{\lambda}(B)<c_{\lambda}:=c\left(I_{\lambda} ; \mathcal{F}\right)$. Then, for almost all $\lambda \in T$, there exists a sequence $\left(x_{n}\right)_{n}$ in $X$ such that: 
(i) $\lim _{n} I_{\lambda}\left(x_{n}\right)=c_{\lambda}$,

(ii) $\lim _{n}\left\|I_{\lambda}^{\prime}\left(x_{n}\right)\right\|=0$ and

(iii) $\left(x_{n}\right)_{n}$ is bounded.

The additional information (iii) leads to a substantial weakening of the PalaisSmale condition required for compactness. Say that a functional satisfies the Weak$P S$ condition (WPS) if any sequence $\left(x_{n}\right)_{n}$ satisfying (i), (ii), (iii) above is relatively compact. In this case, $I_{\lambda}$ will have a critical point at level $c_{\lambda}$ for a dense subset $S$ of $\lambda$ 's in $T$.

Remark 7.1. (1) One cannot expect such bounded (PS)-sequences for all $\lambda$. Indeed, as remarked by $\mathrm{H}$. Brezis, there exists $\varepsilon>0$ such that the family of functions $\left(I_{\lambda}\right)_{\lambda \in[-\varepsilon, \varepsilon]}$ defined on $\mathbf{R}^{2}$ by

$$
I_{\lambda}(x, y)=x^{2}-(x-1)^{3} y^{2}-\lambda\left(x^{2}+y^{2}\right)
$$

satisfies the assumptions of Theorem 7.1 with $\mathcal{F}$ being the mountain pass class, yet there is no bounded Palais-Smale sequence for $I=I_{0}$ at the mountain-pass level.

(2) In the presence of sets $M_{\lambda}$ that are dual to $\mathcal{F}$ in such a way that inf $I_{\lambda}\left(M_{\lambda}\right)=$ $c_{\lambda}$, one can show the existence of an almost critical sequence $\left(x_{n}\right)_{n}$ which is bounded and which also satisfies $\operatorname{dist}\left(x_{n} ; M_{\lambda}\right) \rightarrow 0$.

To prove the theorem, note first that the map $\lambda \rightarrow c_{\lambda}$ is non-increasing and therefore differentiable almost everywhere. We show that $I_{\lambda}$ has a bounded PalaisSmale sequence at each level $c_{\lambda}$ where the derivative $c_{\lambda}^{\prime}$ of $c_{\lambda}$ with respect to $\lambda$ exists.

Let $\lambda \in T$ be such a value and let $\left\{\lambda_{n}\right\} \subset T$ be a strictly increasing sequence such that $\lambda_{n} \uparrow \lambda$. Start with a sequence of sets $A_{n} \in \mathcal{F}$ and a constant $K=K\left(c_{\lambda}^{\prime}\right)>0$ verifying:

$$
\max _{A_{n}} I_{\lambda} \leq c_{\lambda}+\left(2-c_{\lambda}^{\prime}\right)\left(\lambda-\lambda_{n}\right)
$$

and

$$
x \in A_{n} \cap\left\{I_{\lambda} \geq c_{\lambda}-\left(\lambda-\lambda_{n}\right)\right\} \quad \Longrightarrow \quad\|x\| \leq K .
$$

For that, it suffices to choose $A_{n} \in \mathcal{F}$ such that $\max _{A_{n}} I_{\lambda_{n}} \leq c_{\lambda_{n}}+\left(\lambda-\lambda_{n}\right)$. Indeed, if $x \in A_{n} \cap\left\{I_{\lambda} \geq c_{\lambda}-\left(\lambda-\lambda_{n}\right)\right\}$, then

$$
\begin{aligned}
\frac{I_{\lambda_{n}}(x)-I_{\lambda}(x)}{\lambda-\lambda_{n}} \leq & \frac{c_{\lambda_{n}}+\left(\lambda-\lambda_{n}\right)-c_{\lambda}+\left(\lambda-\lambda_{n}\right)}{\lambda-\lambda_{n}} \\
& =\frac{c_{\lambda_{n}}-c_{\lambda}}{\lambda-\lambda_{n}}+2 .
\end{aligned}
$$

Since $c_{\lambda}^{\prime}$ exists, there is $n(\lambda) \in \mathbf{N}$ such that $\forall n \geq n(\lambda)$,

$$
J_{2}(x)=\frac{I_{\lambda_{n}}(x)-I_{\lambda}(x)}{\lambda-\lambda_{n}} \leq-c_{\lambda}^{\prime}+3 .
$$

Also $J_{1}(x)=I_{\lambda_{n}}(x)+\lambda_{n} J_{2}(x) \leq c_{\lambda_{n}}+\left(\lambda-\lambda_{n}\right)+\lambda_{n}\left(-c_{\lambda}^{\prime}+3\right) \leq C$. Using the assumption that $J_{2}(x) \rightarrow+\infty$ as $\|x\| \rightarrow \infty$, one finds that the uniform boundedness of $J_{2}(x)$ proves (7.1). Relation (7.2) then follows from the fact that $I_{\lambda_{n}} \geq I_{\lambda}$ and that for all $n \geq n(\lambda)$, we have $c_{\lambda_{n}} \leq c_{\lambda}+\left(-c_{\lambda}^{\prime}+1\right)\left(\lambda-\lambda_{n}\right)$. 
Roughly speaking, we have shown that there exists a sequence $A_{n} \in \mathcal{F}$ such that $\max _{A_{n}} I_{\lambda} \rightarrow c_{\lambda}$ and for which, if $n \in \mathbf{N}$ is large enough, the higher part of the path $A_{n}$ is contained in the ball of radius $K=K\left(c_{\lambda}^{\prime}\right)>0$. Now for $\alpha>0$, define

$$
F_{\alpha}=\left\{x \in X:\|x\| \leq K+1 \text { and }\left|I_{\lambda}(x)-c_{\lambda}\right| \leq \alpha\right\} .
$$

It is not difficult to show - using the standard deformation lemma - that for all $\alpha>$ $0, \inf \left\{\left\|I_{\lambda}^{\prime}(x)\right\|: x \in F_{\alpha}\right\}=0$. Note that this follows directly from Corollary 3.1, in the case where $I^{\prime}$ is uniformly continuous.

7.2. The superquadraticity condition. In dealing with functionals of the form

$$
I(u)=\frac{1}{2} \int_{\mathbf{R}^{n}}\left(|\nabla u|^{2}+K u^{2}\right) d x-\int_{\mathbf{R}^{n}} F(x, u) d x
$$

one often encounters the following condition $(\mathrm{A}-\mathrm{R})$ :

There is $\mu>2$ such that $0 \leq \mu F(x, s) \leq f(x, s) s$ for all $s \geq 0$ and a.e. $x \in \mathbf{R}^{n}$ where $f(x,$.$) is the derivative of F(x,$.$) . This condition - originally introduced by$ Ambrosetti-Rabinowitz in A-R - usually plays a dual purpose. First, it insures that we are dealing with the following superquadratic nonlinearity (SQC):

$$
f(x, s) \geq C_{1}|s|^{\mu-1}+C_{2} \text { for all } s .
$$

But also it is needed to insure -via the interplay between the functional and its derivative-that Palais-Smale sequences at a given level are actually bounded. It is commonly understood that it is desirable to do away with (A-R) since it is not as natural an assumption as (SQC). We show, in the following example due to Jeanjean [J], how Theorem 7.1 above can help. The example will also give a glimpse at another widely applied technique in the analysis of Palais-Smale sequences: the method of concentration compactness developed by P. L. Lions [L1].

Example (14): A Landesman-Lazer type problem on $\mathbf{R}^{n}$. Consider the problem

$$
\left\{\begin{array}{l}
-\Delta u(x)+K u(x)=f(x, u(x)) \\
u \in H^{1}\left(\mathbf{R}^{n}\right), \quad u>0
\end{array}\right.
$$

where $K>0$ and $n \geq 3$. Without loss, we may assume that $f(x, s)=0, \forall s<0$, a.e. $x \in \mathbf{R}^{n}$, leading to a Landesman-Lazer type problem (i.e., where $f(x, s)$ has different behaviours as $s$ approaches $+\infty$ or $-\infty)$. We consider the following hypothesis on $f$ and its antiderivative $F$ :

(H1) $f: \mathbf{R}^{n} \times \mathbf{R}^{+} \rightarrow \mathbf{R}$ is a Caratheodory function such that $f(., s) \in L^{\infty}\left(\mathbf{R}^{n}\right)$ and $f(., s)$ is 1 -periodic in $x_{i}, 1 \leq i \leq n$.

(H2) There is $p \in\left(2, \frac{2 n}{n-2}\right)$ such that $\lim _{s \rightarrow \infty} f(x, s) s^{1-p}=0$ uniformly in $x \in \mathbf{R}^{n}$.

(H3) $\lim _{s \rightarrow 0} f(x, s) s^{-1}=0$ uniformly in $x \in \mathbf{R}^{n}$.

(H4) There is $a \in(0, \infty]$ such that $\lim _{s \rightarrow+\infty} f(x, s) s^{-1}=a$ uniformly in $x \in \mathbf{R}^{n}$.

Let $G: \mathbf{R}^{n} \times \mathbf{R}^{+} \rightarrow \mathbf{R}$ be defined by $G(x, s)=\frac{1}{2} f(x, s) s-F(x, s)$ and introduce the additional assumptions:

(A1) $G(x, s) \geq 0$ for $s \geq 0$ and almost all $x \in \mathbf{R}^{n}$, and there is $\delta>0$ such that

$$
f(x, s) s^{-1} \geq K-\delta \Longrightarrow G(x, s) \geq \delta \text {. }
$$


(A2) There is $C \in\left[1, \infty\left[\right.\right.$ such that for almost all $x \in \mathbf{R}^{n}$,

$$
G(x, s) \leq C G(x, t), \forall(t, s) \in \mathbf{R}^{+} \times \mathbf{R}^{+} \text {with } s \leq t .
$$

Remark 7.2. If for almost all $x \in \mathbf{R}^{n}, f(x, s) s^{-1}$ is a non-decreasing function of $s \geq 0$, then both (A1) and (A2) are satisfied. In particular, (A2) then holds with $C=1$. Note also that (A2) implies (A1) and thus the assumption on $G$ is weaker when the non-linearity is asymptotically linear. Finally, observe that (H2) always holds when $a<\infty$ in (H4). Here is a result of Jeanjean [J].

Proposition 7.1. Suppose $f$ satisfies (H1)-(H4). Also assume one of the following two conditions:

(i) $a<\infty$ in (H4) and (A1) holds with $K \in(0, a)$.

(ii) $a=\infty$ in (H4) and (A2) holds.

Then, there exists a non-trivial positive solution of (7.6).

Critical points of the functional $I_{\lambda}: H^{1}\left(\mathbf{R}^{n}\right) \rightarrow \mathbf{R}$ defined by

$$
I_{\lambda}(u)=\frac{1}{2} \int_{\mathbf{R}^{n}}\left(|\nabla u|^{2}+K u^{2}\right) d x-\lambda \int_{\mathbf{R}^{n}} F(x, u) d x
$$

are solutions of problem (7.6) when $\lambda=1$. Also by the weak maximum principle, they are positive solutions. Hypotheses (H1)-(H4) imply (when $K \in(0, a)$ ) that $I$ possesses a mountain pass geometry.

In the special case where $f$ is autonomous (i.e., the non-linearity does not depend explicitly on $x \in \mathbf{R}^{n}$ ) the existence of one solution of (7.6) (and even infinitely many) was proved by Berestycki-Lions [Be-L] under the hypotheses (H1)-(H4). To obtain the existence of one solution, they develop a subtle Lagrange multiplier procedure which ultimately relies on a Pohozaev-type identity. The lack of compactness due to the translation invariance of (7.6) is regained by working in the subspace of $H^{1}\left(\mathbf{R}^{n}\right)$ consisting of radially symmetric functions. In the general case where $f$ is not autonomous, Pohozaev's identity provides no information, and usually one assumes (A-R) in addition to (H1)-(H4).

In Proposition 7.1, (A-R) is replaced by (A1) if $a<\infty$ or by (A2) if $a=\infty$ in (H4). Since (A-R) implies that $f(x,$.$) increases at least like s^{\mu-1}$ for $s \rightarrow \infty$, that condition cannot hold when $a<\infty$. On the other hand, when $a=\infty$ it may happen that (A-R) is satisfied but the hypotheses on $f$ do not necessarily imply it. For example (A-R) is not true for the non-linearity $f(x, s)=f(s)=s \ln (s+1)$ for $s \geq 0$ which satisfies (H1)-(H4) and (A2).

To sketch the proof of Proposition 7.1, we start by noticing that the family of functionals is of the form $I_{\lambda}=I_{1}-\lambda I_{2}$ for $\lambda \in[1,2]$ and satisfies the assumptions of Theorem 7.1. Thus, for almost every $\lambda \in[1,2]$ there exists a bounded sequence $\left\{v_{m}\right\} \subset H^{1}\left(\mathbf{R}^{n}\right)$ such that $I_{\lambda}\left(v_{m}\right) \rightarrow c_{\lambda}$ and $I_{\lambda}^{\prime}\left(v_{m}\right) \rightarrow 0$ in $H^{-1}\left(\mathbf{R}^{n}\right)$. Using the translation invariance of (7.6) one constructs a sequence $\left\{y_{m}\right\} \subset \mathbf{Z}^{n}$ such that $u_{m}(x):=v_{m}\left(x-y_{m}\right)$ satisfies $u_{m} \rightarrow u_{\lambda} \neq 0$ weakly in $H^{1}\left(\mathbf{R}^{n}\right)$ with $I_{\lambda}\left(u_{\lambda}\right) \leq c_{\lambda}$ and $I_{\lambda}^{\prime}\left(u_{\lambda}\right)=0$. From the weak maximum principle one gets that $u_{\lambda} \geq 0$ a.e. So far, we have a sequence $\left\{\left(\lambda_{k}, u_{k}\right)\right\} \subset[1,2] \times H^{1}\left(\mathbf{R}^{n}\right)$ with $u_{k} \geq 0$ a.e. such that $\lambda_{k}$ is decreasing to $1, u_{k} \neq 0, I_{\lambda_{k}}\left(u_{k}\right) \leq c_{\lambda_{k}}$ and $I_{\lambda_{k}}^{\prime}\left(u_{k}\right)=0$.

To prove the boundedness of $\left\{u_{k}\right\}$, use the P. L. Lions concentration compactness principle [L1] by assuming that $\left\|u_{k}\right\| \rightarrow \infty$, then setting $w_{k}=u_{k}\left\|u_{k}\right\|^{-1}$ and constructing a subsequence $w_{k} \rightarrow w$ in $H^{1}\left(\mathbf{R}^{n}\right)$ that satisfies one of the two following alternatives. 
(1) (non-vanishing) $\exists \alpha>0, y \in \mathbf{Z}^{n}$ and $R<\infty$ such that $\lim _{k \rightarrow \infty} \int_{y+B_{R}} w_{k}^{2} d x \geq$ $\alpha>0$.

(2) (vanishing) $\lim _{k \rightarrow \infty} \sup _{y \in \mathbf{Z}^{n}} \int_{y+B_{R}} w_{k}^{2} d x=0, \forall R<\infty$.

To eliminate the first alternative where $w \neq 0$, we distinguish the cases $a<\infty$ and $a=\infty$ in (H4). When $a<\infty$ one can show that $w \neq 0$ satisfies $-\Delta w+K w=$ aw on $\mathbf{R}^{n}$, which contradicts the fact that the operator $-\Delta$ has no eigenvector in $H^{1}\left(\mathbf{R}^{n}\right)$. When $a=\infty$, the condition $f(x, s) s^{-1} \rightarrow \infty$ as $s \rightarrow \infty$ for almost all $x \in \mathbf{R}^{n}$ prevents the set $\Omega=\left\{x \in \mathbf{R}^{n} ; w(x)>0\right\}$ from having a non-zero Lebesgue measure which contradicts that $w \neq 0$.

To eliminate the second alternative, one notices first that $\int_{\mathbf{R}^{n}} G\left(x, u_{k}\right) d x \leq$ $\frac{c_{\lambda_{k}}}{\lambda_{k}} \leq c$ for all $k \in \mathbf{N}$. But if $a<\infty$ and (A1) holds, the integral goes to $+\infty$. Finally when $a=\infty$, the vanishing of $\left\{w_{k}\right\}$ is incompatible with the radial behaviour of $I$ which is guaranteed by (A2). Having proved the boundedness of $\left\{u_{k}\right\} \subset H^{1}\left(\mathbf{R}^{n}\right)$, one finds that the proof of the convergence to a non-trivial critical point of $I$ is straightforward.

\section{RECOVERING COMPACTNESS FROM SECOND ORDER INFORMATION}

This method consists of constructing "approximate critical points" with some information on the Hessian at these points that can be useful in establishing their convergence. This program -initiated in full generality by Fang-Ghoussoub in [F-G], G3 - was originally motivated by the work of P. L. Lions on the Hartree-Fock equations [L2, where the associated energy functional $I$ lacks the Palais-Smale condition on its domain $X$. P. L. Lions first noticed that in the search for the steady state, one can use the perturbed minimization principle of Ekeland [E1] to find a sequence $\left(x_{k}\right)_{k}$ that is minimizing $\left(I\left(x_{k}\right) \rightarrow \inf _{X} I\right)$, almost critical $\left(I^{\prime}\left(x_{k}\right) \rightarrow 0\right)$ and with the following additional second order information:

$$
\liminf _{k \rightarrow \infty}\left\langle I^{\prime \prime}\left(x_{k}\right) w, w\right\rangle \geq 0 \text { for all } w \in X
$$

This extra property turned out to be crucial in the proof of convergence of such sequences and therefore in solving the initial minimization problem. Lions then used ad-hoc arguments to extract second order information at higher critical levels so as to establish compactness and therefore the existence of an infinite number of excitable states. Recently, we have come to realize that Taubes had already followed this strategy in his work on the Yang-Mills-Higgs equations [Tau1], [Tau2], [Tau3]. In F-G], G3] general results were obtained in the context of unstable critical points obtained by min-max methods. The existence of Palais-Smale sequences with the appropriate second order information as dictated by the dimension of the minmax class (and therefore by the energy level) was established, provided we have an assumption of Hölder continuity on the first and second derivatives of the functional.

To analyze the higher dimensional analogues of almost minima, define for any $u$ in a Hilbert space $E$ and $\rho>0$, the following approximate Morse indices:

$$
m_{\rho}(u)=\sup \left\{\operatorname{dim}(L) ; L \text { subspace of } E,\left\langle I^{\prime \prime}(u) w, w\right\rangle<-\rho\|w\|^{2}, \forall w \in L\right\}
$$

and

$$
m_{\rho}^{*}(u)=\inf \left\{\operatorname{codim}(L) ; L \text { subspace of } E,\left\langle I^{\prime \prime}(u) w, w\right\rangle>\rho\|w\|^{2}, \forall w \in L\right\} .
$$


Note that if $\rho=0$, then $m_{\rho}(u)$ (resp. $m_{\rho}^{*}(u)$ ) coincide with the Morse index $m(u)$ (resp., the augmented Morse index $m^{*}(u)$ ) of $u$.

8.1. Variational principles with second order information. The following results were established in Fang-Ghoussoub [F-G], G3].

Theorem 8.1. Let $I$ be a $C^{2}$-functional on a Hilbert space $E$ and let $\mathcal{F}$ be a homotopic (resp. a homological) family of dimension $n$ with boundary $B$ and assume that $I^{\prime}$ and $I^{\prime \prime}$ are Hölder continuous around the level $c:=c(I, \mathcal{F})$. Assume $M$ is a closed subset of $E$ such that:

$$
M \text { is dual to } \mathcal{F} \text { and } \inf _{x \in M} I(x) \geq c-\epsilon,
$$

for $\epsilon$ small enough. Then, there exists $\delta(\epsilon)>0$ with $\lim _{\epsilon \rightarrow 0} \delta(\epsilon)=0$ such that for any $A \in \mathcal{F}$ with $\max _{x \in A} I(x) \leq c+\epsilon$, there exists $x_{\epsilon} \in E$ that satisfies:

(i) $c-\epsilon \leq I\left(x_{\epsilon}\right) \leq c+\epsilon$;

(ii) $\left\|I^{\prime}\left(x_{\epsilon}\right)\right\| \leq \delta(\epsilon)$;

(iii) $x_{\epsilon} \in A$;

(iv) $\operatorname{dist}\left(x_{\epsilon}, M\right) \leq \delta(\epsilon)$;

(v) $m_{\delta(\epsilon)}\left(x_{\epsilon}\right) \leq n \quad$ (resp. $\left.m_{\delta(\epsilon)}\left(x_{\epsilon}\right) \leq n \leq m_{\delta(\epsilon)}^{*}\left(x_{\epsilon}\right)\right)$.

The following definitions are now in order:

(1) A $C^{2}$-functional $I$ on a Hilbert space $E$ is said to satisfy $(P S)_{c, n^{-}}$if a sequence $\left(x_{k}\right)_{k}$ in $E$ is relatively compact whenever it satisfies the following conditions: $\lim _{k} I\left(x_{k}\right)=c, \lim _{k}\left\|I^{\prime}\left(x_{k}\right)\right\|=0$, and $m_{\rho_{k}}\left(x_{k}\right) \leq n$ for some sequence $\rho_{k} \downarrow 0$.

(2) It is said to satisfy $(P S)_{c, n}$ if in the above definition, $\left(x_{k}\right)_{x}$ is also assumed to satisfy $m_{\rho_{k}}\left(x_{k}\right) \leq n \leq m_{\rho_{k}}^{*}\left(x_{k}\right)$ for some sequence $\rho_{k} \downarrow 0$.

8.2. Compactness via upper estimates on approximate Morse indices. Upper estimates -on the approximate indices- in the context of higher dimensional homotopic classes were also used by Taubes in the context of the Yang-MillsHiggs equations [Tau1, Tau2], Tau3] and by Esteban-Séré [E-S] in their work on the Dirac-Fock equations. We shall describe below the simpler settings of the Hartree-Fock equations mentioned above as well as the problem of existence of closed geodesics on $S^{N} \times \mathbf{R}$ discussed in Section 2 .

Example (15): The closed geodesics problem revisited. Looking back at Proposition 2.6 which analyzes the behaviour of Palais-Smale sequences, we can now add the following to the properties of the associated functional $I$ on $\Lambda$.

(1) $I$ satisfies $(P S)_{c}$ for any $c \in \mathbf{R} \backslash\left\{2 \pi^{2} k^{2} ; k=1,2, \ldots\right\}$.

(2) For each $k \geq 2, I$ satisfies $(P S)_{c, n^{-}}$for $c=2 \pi^{2} k^{2}$ and $n=N-1$.

Indeed, in the case where $\left(u_{j}\right)_{j=1}^{\infty}=\left(s_{j}, x_{j}\right)_{j=1}^{\infty} \subset \Lambda$ is a Palais-Smale sequence such that $I\left(u_{j}\right) \rightarrow 2 \pi^{2} k^{2}$, then by Proposition 2.6, $\tilde{u}_{j}(t) \equiv\left(s_{j}(t)-s_{j}(0), x_{j}(t)\right) \rightarrow$ $\left(0, y_{k}(t)\right)$ in $\Lambda$ as $j \rightarrow \infty$, where $y_{k}(t)$ is given by $y_{k}(t)=e_{1} \cos 2 \pi k t+e_{2} \sin 2 \pi k t$ and $e_{1}, \cdots, e_{N} \in \mathbf{R}^{N}$ is an orthonormal basis of $\mathbf{R}^{N}$. Since $I^{\prime \prime}\left(u_{j}\right) \rightarrow I_{0}{ }^{\prime \prime}\left(0, y_{k}\right)$ where $I_{0}$ is the functional associated to the standard metric $g^{0}$, one notes that the Morse index of $I_{0}$ at $\left(0, y_{k}\right)$ is larger than $(N-2)(2 k-1)$. This can be easily seen by noticing that $I_{0}{ }^{\prime \prime}\left(0, y_{k}\right)((0, v),(0, v))<0$ for all $v \in V \backslash\{0\}$, where $V$ is spanned by $e_{i} \cos 2 \pi j t$ and $e_{i} \sin 2 \pi j t$ for $i=3,4, \cdots, N$ and $j=0,1, \cdots k-1$, which has 
dimension $(N-2)(2 k-1)$. Assertion (2) follows because the lack of compactness of $\left(u_{j}\right)_{j}$ would then imply:

$$
(N-2)(2 k-1) \leq \liminf _{j \rightarrow \infty} m_{\delta_{j}}\left(u_{j}\right) \leq N-1 .
$$

Now, we can deal with the unfinished business in that example. That is when $\bar{b}=2 \pi^{2} k_{0}^{2}$ with $k_{0}=2,3, \cdots$. Choose $L_{0} \geq 2$ such that $I\left(r, \sigma_{0}(z)\right) \leq 3 \pi^{2}$ for all $|r| \geq L_{0}$ and $z \in S^{N-2}$, and set

$$
\begin{gathered}
\bar{\Gamma}_{0}=\left\{\gamma \in C\left(\left[-L_{0}, L_{0}\right] \times S^{N-2}, \Lambda\right) ; \gamma\left( \pm L_{0}, z\right)=\left( \pm L_{0}, \sigma_{0}(z)\right)\right\}, \\
\bar{b}_{0}=\inf _{\gamma \in \bar{\Gamma}_{0}(r, z) \in\left[-L_{0}, L_{0}\right] \times S^{N-2}} E(\gamma(r, z)) .
\end{gathered}
$$

One easily checks that $\bar{b}_{0}=\bar{b}$, which means that $\bar{b}$ can be achieved by a homotopic family of dimension $(N-1)$. In view of Theorem 8.1 above, the remaining case of Proposition 2.8 is also settled.

Example (16): The Hartree-Fock equations. Consider the purely Coulombic $N$-body Hamiltonian

$$
H=-\sum_{i=1}^{N} \Delta_{x_{i}}+\sum_{i=1}^{N} V\left(x_{i}\right)+\sum_{i<j} \frac{1}{\left|x_{i}-x_{j}\right|}
$$

where $V(x)=-\sum_{j=1}^{m} z_{j}\left|x-\bar{x}_{j}\right|^{-1}, m \geq 1, z_{j}>0, \bar{x}_{j} \in \mathbf{R}^{3}$ are fixed. Write $Z=\sum_{j=1}^{m} z_{j}$ for the total charge of the nuclei. We are looking for the critical points of the functional

$$
\begin{aligned}
I\left(u_{1}, \ldots, u_{N}\right)= & \sum_{i=1}^{N} \int_{\mathbf{R}^{3}}\left|\nabla u_{i}\right|^{2}+V\left|u_{i}\right|^{2} d x \\
& +\frac{1}{2} \iint_{\mathbf{R}^{3} \times \mathbf{R}^{3}} \varrho(x) \frac{1}{|x-y|} \varrho(y) d x d y \\
& -\frac{1}{2} \iint_{\mathbf{R}^{3} \times \mathbf{R}^{3}} \frac{1}{|x-y|}|\varrho(x, y)|^{2} d x d y
\end{aligned}
$$

over the manifold

$$
M=\left\{\left(u_{1}, \ldots, u_{N}\right) \in H^{1}\left(\mathbf{R}^{3}\right)^{N} ; \int_{\mathbf{R}^{3}} u_{i} u_{j}^{*} d x=\delta_{i j}\right\}
$$

where $z^{*}$ denotes the conjugate of the complex $z$, while $\varrho(x)=\sum_{i=1}^{N}\left|u_{i}\right|^{2}(x)$ is the density and $\varrho(x, y)=\sum_{i=1}^{N} u_{i}(x) u_{i}^{*}(y)$ is the density matrix associated to $\left(u_{1}, \ldots, u_{n}\right)$.

The Euler-Lagrange equations corresponding to the above problem (after a suitable diagonalization) are the following so-called Hartree-Fock equations: For $1 \leq i \leq N$,

$$
-\Delta u_{i}+V u_{i}+\left(\rho * \frac{1}{|x|}\right) u_{i}-\int_{\mathbf{R}^{3}} \rho(x, y) \frac{1}{|x-y|} u_{i}(y) d y+\epsilon_{i} u_{i}=0
$$

where for each $i, \lambda_{i}=-\epsilon_{i}$ is the Lagrange multiplier and $\left(u_{1}, \ldots, u_{N}\right) \in M$. Here is a result of P. L. Lions [L2].

Proposition 8.1. If $Z>N$, then the Hartree-Fock equations (8.4) have infinitely many solutions. 
One first shows that the functional $I$ restricted to $M$ verifies $(P S)_{c, k^{-}}$for every $c \in \mathbf{R}$ and any $k \in \mathbf{N}$. Indeed, let $\left(u^{n}\right)_{n}=\left(u_{1}^{n}, \ldots, u_{N}^{n}\right) \in M$ be a sequence such that $\left(I\left(u^{n}\right)\right)_{n}$ is bounded and

$$
\left(I_{\mid M}\right)^{\prime}\left(u^{n}\right) \rightarrow 0 \text { while }\left\langle\left(I_{\mid M}\right)^{\prime \prime}\left(u^{n}\right) v, v\right\rangle \geq-\gamma^{n}\|v\|^{2} \text { for every } v \text { in } E_{n},
$$

where $E_{n}$ is a subspace of $T_{u^{n}}(M)$ of codimension at most $k$ and where $\left(\gamma_{n}\right)_{n}$ is a sequence of scalars decreasing to 0 . The first and second derivative conditions yield the existence of $\left(\varepsilon_{1}^{n}, \ldots, \varepsilon_{N}^{n}\right) \in \mathbf{R}^{N}$ such that the following holds in $L^{2}\left(\mathbf{R}^{3}\right)$ :

$$
\lim _{n}\left(-\Delta u_{i}^{n}+V u_{i}^{n}+\left(\varrho^{n} * \frac{1}{|x|}\right) u_{i}^{n}-\int_{\mathbf{R}^{3}} \varrho^{n}(x, y) u_{i}^{n}(y) \frac{1}{|x-y|} d y+\varepsilon_{i}^{n} u_{i}^{n}\right)=0,
$$

and for each fixed $i$,

$$
\int_{\mathbf{R}^{3}}\left(|\nabla w|^{2}+V|w|^{2}+\left(\varrho^{n} * \frac{1}{|x|}\right)|w|^{2}\right) d x+\left(\varepsilon_{i}^{n}+\gamma^{n}\right) \int_{\mathbf{R}^{3}}|w|^{2} d x \geq 0
$$

for all $w$ in a closed subspace of $H^{1}\left(\mathbf{R}^{3}\right)$ of codimension at most $k+N$.

We now use this second order information to find lower bounds for $\varepsilon_{i}^{n}$. Indeed, (8.7) implies that the Schrödinger operator $H_{n}=-\Delta+V+\varrho^{n} * \frac{1}{|x|}$ has at most $k+N$ eigenvalues less than $-\left(\varepsilon_{i}^{n}+\gamma^{n}\right)$. On the other hand, we have the following classical lemma (see [L2]).

Lemma 8.1. Assume that $\mu$ is a bounded non-negative measure on $\mathbf{R}^{3}$ such that $\mu\left(\mathbf{R}^{3}\right)<Z$ and let $H_{\mu}$ be the Hamiltonian given by $H_{\mu}=-\Delta+V+\mu * \frac{1}{|x|}$. Then, for each integer $k$, there is $\delta>0$ such that $H_{\mu}$ admits at least $k$ eigenvalues strictly below $-\delta$.

Back to the proof of the proposition. Since $Z>N$, the lemma yields a $\delta>0$ such that $H_{n}$ admits for all $n$ at least $k+N$ eigenvalues below $-\delta$. It follows that $\varepsilon_{i}^{n}+\gamma^{n} \geq \delta$ which means that for $n$ large enough, we have for every $i$,

$$
\varepsilon_{i}^{n} \geq \delta / 2>0
$$

Now note that the Palais-Smale sequence $\left(u^{n}\right)_{n}$ is bounded in $H^{1}\left(\mathbf{R}^{3}\right)^{N}$. This can be seen by combining the fact that $I\left(u_{1}^{n}, \ldots, u_{N}^{n}\right)$ is bounded with the CauchySchwarz inequalities

$$
|\rho(x, y)|^{2} \leq \rho(x) \rho(y) \text { on } \mathbf{R}^{3} \times \mathbf{R}^{3},
$$

and the following estimate:

$$
\int_{\mathbf{R}^{3}} \frac{1}{|x-\bar{x}|}|u(x)|^{2} d x \leq C\|u\|_{L^{2}\left(\mathbf{R}^{3}\right)}\|\nabla u\|_{L^{2}\left(\mathbf{R}^{3}\right)}
$$

which holds for some $C$ independent of $\bar{x} \in \mathbf{R}^{3}$ and $u \in H^{1}\left(\mathbf{R}^{3}\right)$. We can also deduce that $\varepsilon_{i}^{n}$ is bounded, and thus we may assume - by extracting subsequences if necessary - that $u_{i}^{n}$ converges weakly in $H^{1}\left(\mathbf{R}^{3}\right)$ (and a.e. in $\mathbf{R}^{3}$ ) to some $u_{i}$ and that $\varepsilon_{i}^{n}$ converges to $\varepsilon_{i}$ which satisfies $\varepsilon_{i} \geq \delta / 2>0$. Passing to the limit in (8.6), we get

$$
-\Delta u_{i}+V u_{i}+\left(\varrho * \frac{1}{|x|}\right) u_{i}+\varepsilon_{i} u_{i}-\int_{\mathbf{R}^{3}} \varrho(x, y) \frac{1}{|x-y|} u_{i}(y) d y=0
$$


and

$$
\begin{aligned}
\lim \sup _{n} \sum_{i} \varepsilon_{i}^{n} \int_{\mathbf{R}^{3}}\left|u_{i}^{n}\right|^{2} d x= & -\liminf _{n}\left\{\sum_{i} \int_{\mathbf{R}^{3}}\left(\left|\nabla u_{i}^{n}\right|^{2} \quad+V\left|u_{i}^{n}\right|^{2}\right) d x\right. \\
& \left.+\iint_{\mathbf{R}^{3} \times \mathbf{R}^{3}}\left(\varrho^{n}(x) \varrho^{n}(y)-\left|\varrho^{n}(x, y)\right|^{2}\right) \frac{1}{|x-y|} d x d y\right\} \\
\leq & -\left\{\sum_{i} \int_{\mathbf{R}^{3}}\left(|\nabla u|^{2}+V\left|u_{i}\right|^{2}\right) d x\right. \\
& \left.+\iint_{\mathbf{R}^{3} \times \mathbf{R}^{3}}\left(\varrho(x) \varrho(y)-|\varrho(x, y)|^{2}\right) \frac{1}{|x-y|} d x d y\right\} \\
= & \sum_{i} \varepsilon_{i} \int_{R^{3}}\left|u_{i}\right|^{2} d x .
\end{aligned}
$$

Hence $\lim _{n}\left\|u_{i}^{n}\right\|_{2}=\left\|u_{i}\right\|_{2}$ for every $i$, and consequently, $u_{i}^{n}$ converges in $L^{2}\left(\mathbf{R}^{3}\right)$ to $u_{i}$. This concludes the proof that $I$ satisfies $(P S)_{c, k^{-}}$.

To set up the min-max principles, consider for each $k \in \mathbf{N}$ the following $k$ dimensional $\mathbf{Z}_{2}$-homotopy stable family $\mathcal{F}_{k}=\left\{A ; A=f\left(S^{k-1}\right), f: S^{k-1} \rightarrow M\right.$ odd and continuous $\}$. Letting $c_{k}=c\left(I, \mathcal{F}_{k}\right)$, we have

$$
-\infty<c_{k} \leq c_{k+1}<0 \text { for each } k \in \mathbf{N} \text { and } \lim _{k} c_{k}=0 .
$$

Indeed, $-\infty<c_{k}$ because $I$ is bounded below on $M$. Lemma 8.1 yields the existence of a $k$-dimensional subspace $V_{k}$ of $H^{1}\left(\mathbf{R}^{3}\right)$ such that for all $u \in V_{k}$ with $\|u\|_{2}=1$, we have $\int_{\mathbf{R}^{3}}|\nabla u|^{2}+V|u|^{2} d x \leq-\nu$ for some $\nu>0$. Such a sphere is homeomorphic to $S^{k-1}$ and is contained in $M$. This implies that $c_{k}<0$, and therefore we can find $A_{k} \in \mathcal{F}_{k}$ such that

$$
c_{k} \leq \max _{A_{k}} I<c_{k} / 2 .
$$

Now consider a nested sequence $E_{k}$ of finite dimensional subspaces of $H^{1}\left(\mathbf{R}^{3}\right)^{N}$ such that $\operatorname{dim}\left(E_{k}\right)=k$ and $\cup_{k} E_{k}$ is dense in $H^{1}\left(\mathbf{R}^{3}\right)^{N}$. Consider the orthogonal complement $F_{k}$ of $E_{k-1}$ which is necessarily dual to the class $\mathcal{F}_{k}$; hence we can find $v_{k} \in A_{k} \cap F_{k}$. Note that $I\left(v_{k}\right) \leq c_{k} / 2<0=I(0)$ and $v_{k} \rightarrow 0$ weakly. Since $I$ is weakly lower semi-continuous, we obtain $0=I(0) \leq \liminf _{k} I\left(v_{k}\right) \leq 0$ which implies that $\lim _{k} c_{k}=0$. Proposition 8.1 now follows from the above and Theorem 8.1.

Remark 8.1. For the Dirac-Fock equations, one replaces the Laplacian with the Dirac operator. Recently, Esteban and Séré $[\mathrm{E}-\mathrm{S}]$ established an analoguous result for these equations but under the condition that $\alpha \max \{Z, 3 N-1\}<\frac{2}{\pi / 2+2 / \pi}<$ $Z+1$ where $\alpha$ is a dimensionless physical constant. They use second order information (Theorem 8.1 above) to establish the compactness of Palais-Smale sequences. However, many more problems are open in the D-F case, not the least of which being that the condition used above by Séré-Esteban is quite restrictive. Indeed, with $\alpha \sim 1 / 137$ and $Z$ an integer, the conditions become $Z \leq 124, N \leq 41$ and $N \leq Z$. This obviously does not cover all known physical possibilities.

\section{TOWARDS A $C^{0}$-THEORY OF CRITICAL POINTS}

While the notions of minimum and maximum of a functional are purely topological, the classical Morse classification of saddle-type critical points involves in 
a crucial way the differential structure of the functional and of its domain. In recent years, many functionals associated to various important variational problems lacked the smoothness properties that are normally needed for the application of the classical theory. For example, $W^{1,2}(M, N)$ is not a Banach manifold when $M$ is a manifold of dimension larger than 2 , and this usually complicates the variational approach for constructing harmonic maps between $M$ and $N$ by finding critical points of the energy functional. Another example is the $C^{1}$ but not $C^{2}$ dual functional associated to a Hamiltonian system E2. Partly, to deal with this kind of difficulty, Hofer $[\mathrm{H}]$ isolated the purely topological notion of a critical point of mountain pass type in order to analyze saddle points obtained in the mountain pass theorem for functionals that fail to be $C^{2}$. In the case of a (smooth) Morse function, these points would have had a Morse index at most one. There are also purely topological (non-smooth) analogues corresponding to higher Morse indices. These were developed by Fang $[\mathrm{F}$ ) and will be summarized later on in this section.

Before that, we mention another reason to consider a $C^{0}$-theory. Given a class of compact subsets $\mathcal{F}$ in $X$, it is easy to see that the function $I \mapsto c(I, \mathcal{F}):=$ $\inf _{A \in \mathcal{F}} \max _{A} I$ is continuous in the $C^{0}$ topology, i.e.,

$$
\max _{x \in X}\|I(x)-J(x)\| \rightarrow 0 \Rightarrow|c(I, \mathcal{F})-c(J, \mathcal{F})| \rightarrow 0 .
$$

On the other hand a $C^{0}$-perturbation on $I$ wreaks havoc on the equation $I^{\prime}(x)=0$. Even a $C^{1}$-perturbation can destroy a critical value if the corresponding critical points are degenerate. The preceding observations tell us that critical values obtained by min-max do enjoy very special stability properties. This simple idea has been very fruitful in the theory of Hamiltonian systems and is at the basis of the emergence of symplectic topology that we discuss in the next section. For now, we describe the extent to which the calculus of variations in the large is a $C^{0}$-theory as opposed to a $C^{1}$-theory (resp., $C^{2}$-theory) for the standard existence theory (resp., for the Morse classification).

Topological definition of critical points. We now assume that $X$ is simply a complete metric space -unless otherwise explicitly specified. Introduce the following notion of "derivative" for a continuous function that was already considered by Morse himself. See also [C-D-M].

Definition 9.1. Let $I: X \rightarrow \mathbf{R}$ be a continuous function and $u \in X$. Denote by $|d I|(u)$ the supremum of the $\sigma$ 's in $[0, \infty)$ such that there exist $\delta>0$ and a continuous deformation $\mathcal{H}: B(u, \delta) \times[0, \delta] \rightarrow X$ satisfying for all $(v, t) \in B(u, \delta) \times$ $[0, \delta]:$

$$
\operatorname{dist}(\mathcal{H}(v, t), v) \leq t \quad \text { and } \quad I(\mathcal{H}(v, t))-I(v) \leq-\sigma t .
$$

The extended real number $|d I|(u)$ is called the weak slope of $I$ at $u$. The latter will be called critical if $|d I|(u)=0$. If $X$ is a $C^{1}$-Finsler manifold and $I$ is a $C^{1}$-function, then $|d I|(u)=\left\|I^{\prime}(u)\right\|$ which amply justifies the definitions. Before considering general min-max principles, one can easily check that with this notion, Ekeland's perturbed minimization principle reads as follows:

Proposition 9.1. Let $I$ be a bounded below continuous functional on a complete metric space $X$. Then, for any minimizing sequence $\left(y_{n}\right)_{n}$, there exists a minimizing sequence $\left(x_{n}\right)_{n}$ such that $\operatorname{dist}\left(x_{n}, y_{n}\right) \rightarrow 0$ and $|d I|\left(x_{n}\right) \rightarrow 0$. 
9.1. Structure of the critical set in the mountain pass theorem. Assume that the complete metric space $X$ is contractible and locally connected. If $u, v \in X$, then a closed subset $F$ of $X$ is dual to $\mathcal{F}_{v}^{u}$ if it separates $u$ and $v$, i.e, if $u, v$ do not belong to the same connected component of $X \backslash F$. To classify the various types of critical points, we use the following notation:

$M_{c}=\left\{x \in K_{c} ; x\right.$ is a local min. of $\left.I\right\}, P_{c}=\left\{x \in K_{c} ; x\right.$ is a proper local max. of $I\}$; that is $x \in P_{c}$ if it is a local maximum of $I$ and if $x \notin \overline{I^{c}}$.

Let $S_{c}=\left\{x \in K_{c} ; x\right.$ is a saddle point of $\left.I\right\}$; that is $x \in S_{c}$ if in each neighborhood of $x$ there exist two points $y$ and $z$ such that $I(y)<I(x)<I(z)$.

Following Hofer $[\mathrm{H}]$, say that a point $x$ in $K_{c}$ is of mountain-pass type if for any neighborhood $N$ of $x$, the set $N \cap I^{c}$ is neither empty nor path connected. We denote by $H_{c}$ the set of critical points of mountain-pass type at level $c$.

Finally, say that 2 disjoint sets $A_{1}$ and $A_{2}$ are connected through a third set $C$ if there is no relatively closed and open subset $F$ of $A_{1} \cup A_{2} \cup C$ that contains one of the $A_{i}$ while being disjoint from the other. The following is due to Fang $[\mathrm{F}$. It extends various results of Pucci-Serrin and Ghoussoub-Preiss (see the references in G2]).

Theorem 9.1. Let $I: X \rightarrow \mathbf{R}$ be a continuous function on $X$. Take two points $u$ and $v$ in $X$ such that $\max \{I(u), I(v)\}<c=c\left(I, \mathcal{F}_{v}^{u}\right)$. If I verifies $(P S)_{c}$, then one of the following three assertions must hold:

(i) $P_{c}$ contains a compact subset that is dual to $\mathcal{F}_{u}^{v}$.

(ii) $K_{c}$ contains a saddle point of mountain-pass type.

(iii) There are finitely many components of $I^{c}$, say $C_{i}(i=1 \cdots, n)$, such that $S_{c}=\cup_{i=1}^{n} S_{c}^{i}$ and $S_{c}^{i} \cap S_{c}^{j}=\emptyset(1 \leq i \neq j \leq n)$ where $S_{c}^{i}=S_{c} \cap \overline{C_{i}}$. Moreover, there are at least two of them, $S_{c}^{i}, S_{c}^{j}(1 \leq i \neq j \leq n)$, such that the sets $\overline{M_{c}} \cap S_{c}^{i}, \overline{M_{c}} \cap S_{c}^{j}$ are non-empty and connected through $M_{c}$.

Note that (i) cannot occur if for instance $X$ is an infinite dimensional Banach space. The theorem then implies that either $K_{c}$ contains a saddle point of mountainpass type or $\overline{M_{c}} \backslash M_{c} \neq \emptyset$. In both cases, we obtain a saddle point. Let's give a quick proof of this fact to illustrate the role of duality. Let $F$ be the boundary of $\{I \geq c\}$. It is clearly dual to the class $\mathcal{F}_{u}^{v}$. By a classical topology result, $F$ contains a closed connected subset $\hat{F} \subset F$ that also separates $u$ and $v$; i.e. $\hat{F}$ is also dual to $\mathcal{F}_{u}^{v}$. Note that $F \cap M_{c}$ is necessarily empty so that if $F \cap S_{c}$ is also empty, then $\hat{F} \cap K_{c}=\hat{F} \cap P_{c}$ and the latter is relatively open in $\hat{F}$ while $\hat{F} \cap K_{c}$ is closed. Since $\hat{F}$ is connected, then either $\hat{F} \cap P_{c}=\emptyset$ or $\hat{F} \cap P_{c}=\hat{F}$. But the first case is impossible since we have $\hat{F} \cap P_{c}=\hat{F} \cap K_{c} \neq \emptyset$ by Theorem 3.1. Hence $\hat{F} \subset P_{c}$ is a compact set separating $u$ and $v$, which is impossible since $X$ is infinite dimensional.

Here is an interesting application of the above.

Corollary 9.1. Suppose I has a local maximum and a local minimum on a Banach space $X$. If I satisfies $(P S)_{c}$ for any $c$ and if $\operatorname{dim}(X) \geq 2$, then necessarily $I$ has a third critical point.

Example (17): Minimal periods for convex Hamiltonian systems. We go back to the problem of finding periodic solutions for Hamiltonian systems. We are 
given a smooth convex function $H: \mathbf{R}^{2 n} \mapsto \mathbf{R}$ and we want to solve the boundaryvalue problem:

$$
\begin{cases}\dot{x} & =J H^{\prime}(x) \\ x(0) & =x(T)\end{cases}
$$

where $J$ is antisymmetric and $J^{*} J=-I$. The first general result concerning this problem is due to Rabinowitz [R4, who showed that problem (9.1) has a non-zero solution provided there is some number $\alpha>2$ such that $0 \leq \alpha H(x) \leq\left(x, H^{\prime}(x)\right)$ for large $|x|$. His method yields no information on the minimal period of this solution, which might be much smaller than $T$. The question was answered much later, by Ekeland and Hofer E-H1.

Proposition 9.2. Assume $H$ is convex, $H(x)>H(0)=0$ for all $x \neq 0$, and that for some $\alpha>2, \omega>0, r>0$ and $R>0$, we have:

$$
\begin{gathered}
\sup _{\|x\| \leq r} H(x)\|x\|^{-2} \leq \omega / 2, \\
H(\lambda x) \geq \lambda^{\alpha} H(x) \text { for }\|x\| \geq R \text { and } \lambda \geq 1, \\
\limsup _{\|x\| \rightarrow \infty} H(x)\|x\|^{-\alpha}<\infty .
\end{gathered}
$$

Then, for every $T<2 \pi / \omega$, problem (9.1) has a solution with minimal period $T$.

To prove the existence of a $T$-periodic solution, define as in example (6):

$$
I(v)=\int_{0}^{T} \frac{1}{2}(J v, \Pi v) d t+\int_{0}^{T} G(-v) d t,
$$

where $v \in V \subset L^{\beta}\left(S^{1} ; \mathbf{R}^{2 n}\right)$ has mean zero, $\beta$ is the conjugate of $\alpha, \frac{d \Pi(v)}{d t}=v(t)$ and $\int_{0}^{1}(\Pi v)(t) d t=0$. The Fenchel transform $G$ of $H$ satisfies dual relations to (9.2), (9.3), (9.4); roughly speaking, it behaves like $\frac{1}{2 \omega}\|x\|^{2}$ near zero, and like $\|x\|^{\beta}$ at infinity. Using these estimates it is not difficult to show that $I$ has a strict local minimum at the origin, with $I(0)=0$, and that there is some function $v$ such that $I(h v) \rightarrow-\infty$ when $h \rightarrow \infty$. The functional $I$ still satisfies the Palais-Smale condition, so the mountain pass theorem yields a non-zero critical point $\bar{v}$ of $I$, corresponding to a $T$-periodic solution $\bar{x}$ of the problem.

By Theorem 9.1, a suitable choice of $\bar{v}$ will leave us with two possibilities: either $\bar{v}$ is a saddle-point of mountain-pass type, or it is a saddle-point that is a limit of a sequence of local minima $\left(v_{n}\right)_{n}$ such that $I\left(v_{n}\right)=I(\bar{v})$.

We will now try to get some information on the topology of the level sets from another source. The obvious place to look is the quadratic form $Q$ on $V$ defined by:

$$
(Q v, v)=\int_{0}^{1} \frac{1}{2}(J v, \Pi v) d t+\int_{0}^{1} \frac{1}{2}\left(G^{\prime \prime}(-\bar{v}(t)) v(t), v(t)\right) d t .
$$

It is not difficult to see that $Q_{x}$ is a compact perturbation of a positive definite quadratic form (the second term on the right) so that it has finite index $m$ (the number of negative terms in an orthogonal splitting). Unfortunately, the function $I$ is not $C^{2}$, so $m$ is not the Morse index of $I$ at $\bar{v}$, and we cannot apply the standard $C^{2}$ tools. The regularity of $H$ or $G$ is not in question; it is simply the fact that, for $\beta \leq 2$, there are no $C^{2}$-functionals on $L^{\beta}$ except quadratic ones. 
To overcome this difficulty, consider the subspace $V_{\infty}=L^{\infty} \cap V$ and the restriction $I_{\infty}$ and $Q_{\infty}$ of $I$ and $Q$ to $V_{\infty}$. It is an easy regularity result that all critical points of $I$ belong to $V_{\infty}$. So $I_{\infty}$ is $C^{2}, \bar{v} \in V_{\infty}$ is a critical point of $I_{\infty}$, and the Hessian of $I_{\infty}$ at $\bar{v}$ is $Q_{\infty}$. It is not obvious (but true) that $\bar{v}$ as a critical point of $I_{\infty}$ inherits the topological properties of $\bar{v}$ as a critical point of $I$ : if $\bar{v}$ is a local minimum, isolated critical point, saddle point of mountain-pass type of $I$, then it is also a local minimum, isolated critical point, saddle point of mountain-pass type of $I_{\infty}$. Here and later, we refer to [E2], section IV.3, for details.

We are now in a $C^{2}$ (but not Hilbertian) setting. If $\bar{v}$ is a limit of local minima, then its Morse index must be zero: $m=0$. If $\bar{v}$ is a saddle-point of mountain-pass type, its Morse index is at most one. If $m=1$, then $\{I<I(\bar{v})\}$ has exactly two path components, $\mathcal{P}_{1}$ and $\mathcal{P}_{2}$, and there is an eigenvector $w$ of $Q$ associated with the negative eigenvalue, such that $\bar{v}+\epsilon w \in \mathcal{P}_{1}$ and $\bar{v}-\epsilon w \in \mathcal{P}_{2}$ for small enough $\epsilon>0$.

Because of the $S^{1}$-invariance of $I$, critical points cannot be isolated: for any $\theta \in S^{1}, T_{\theta} \bar{v}$ is again a critical point. So $Q$ must be degenerate: differentiating with respect to $\theta$ the equation $I_{\infty}^{\prime}\left(T_{\theta} \bar{v}\right)=0$, we find that $\frac{d}{d t} \bar{v} \in \operatorname{Ker} Q_{\infty}$. Assume now that $\bar{v}$ is $1 / k$-periodic for some integer $k \geq 1$. In that case, the $k$ vectors $w_{1}, \ldots, w_{k}$ defined by $w_{i}(t)=\frac{d}{d t} \bar{v}(t)$ for $t \in\left(\frac{i-1}{k}, \frac{i}{k}\right)$ and 0 elsewhere generate a $k$-dimensional subspace on which $Q_{\infty}$ is zero. From this, it is one small step to show that $Q_{\infty}$ has index at least $m \geq k-1$ (and nullity at least 1 ). If $m=0$, then $k=1$ and we have found a solution with minimal period $T$. If $m=1$, then $k=1$ or 2 .

Let us eliminate the case $k=2$. Since $m=1$, it can be checked that the eigenvector $w$ associated with the negative eigenvalue is a $T / 2$-antiperiodic function, i.e. $w(t+T / 2)+w(t)=0$. Without loss of generality, assume that $0 \in \mathcal{P}_{1}$, and connect it with $\bar{v}+\epsilon w$ by a continuous path $c_{1}:[0,1] \mapsto V_{\infty}$ such that $c_{1}(0)=0$ and $c_{1}(1)=\bar{v}+\epsilon w$. Apply a $T / 2$ phase shift to the entire path, thereby defining a new path $c_{2}$ such that $c_{2}(0)=0$ and $c_{2}(1)=\bar{v}-\epsilon w$. Since $k=2, \bar{v}(t)$ is $T / 2$-periodic, and so is $G^{\prime \prime}(\bar{v}(t))$ : the phase shift does not affect the value of the integral. We have $I_{\infty}\left(c_{1}(t)\right)=I_{\infty}\left(c_{2}(t)\right)<I(\bar{v})$ for all $t$, so that $\bar{v}+\epsilon w$ and $\bar{v}-\epsilon w$ belong to the same path component of $\{I<I(\bar{v})\}$, which contradicts the definition of $\mathcal{P}_{1}$ and $\mathcal{P}_{2}$ and concludes the proof.

In later work, Coti Zelati-Ekeland-P.L. Lions C-E-L] (or E2], IV.5) have done away with the growth assumption on $H$ at infinity, at the price of increased smoothness.

Proposition 9.3. Assume that $H(x) \geq H(0)=0$ for all $x$, that the Hessian $H^{\prime \prime}(x)$ is positive definite at every $x \neq 0$, and that $H^{\prime \prime}(x)^{-1} \rightarrow 0$ when $\|x\| \rightarrow \infty$. Assume moreover that $H(x) \rightarrow \infty$ when $\|x\| \rightarrow \infty$, and there is some $\omega>0$ and $r>0$ such that:

$$
\sup _{\|x\| \leq r} H(x)\|x\|^{-2}<\omega / 2
$$

Then, for every $T<2 \pi / \omega$, the boundary-value problem (9.1) has a solution with minimal period $T$.

Choose $h$ so large that $H^{\prime \prime}(x) \geq \frac{2 \pi}{T} I$ whenever $H(x) \geq h$. Set $\Omega:=\{x \mid H(x)<h\}$. Then $\Omega$ is an open, convex and bounded set, and we can find a $C^{2}$-function $\tilde{H}$ such that $H$ and $\tilde{H}$ coincide on $\Omega, \tilde{H}^{\prime \prime}(x)>\frac{2 \pi}{T} I$ whenever $x \notin \Omega$, and $\tilde{H}(x)=\|x\|^{4}$ for $\|x\| \geq R$, where $R$ is some large constant. Applying Proposition 9.2 to $\tilde{H}$, we find 
a solution of the problem:

$$
\begin{cases}\dot{x} & =J \tilde{H}^{\prime}(x) \\ x(0) & =x(T)\end{cases}
$$

with minimal period $T$. We know that $\tilde{H}(x(t))=: \tilde{h}$ is a constant. If $\tilde{h} \geq h$, then $\tilde{H}^{\prime \prime}(x(t))>\frac{2 \pi}{T} I$ for all $t$, so $\tilde{G}^{\prime \prime}(x(t))<\frac{T}{2 \pi} I$, where $\tilde{G}$ is the Fenchel transform of $\tilde{H}$, and it follows easily that the quadratic form

$$
(Q v, v)=\int_{0}^{1} \frac{1}{2}(J v, \Pi v) d t+\int_{0}^{1} \frac{1}{2}\left(\tilde{G}^{\prime \prime}(-\dot{x}(t)) v(t), v(t)\right) d t
$$

has index at least $2 n$. But $\bar{v}:=\dot{x}$ has been shown to have index 0 or 1 . So this is impossible, and therefore $\tilde{h}<h$. But then, the solution $x(t)$ lies entirely in $\Omega$, where $H=\tilde{H}$, and so it is also a solution of the original boundary value problem.

9.2. Classification of higher dimensional saddle points. In this section, we investigate analogues of the Morse indices in a non-smooth setting. Group actions will not be considered here and to avoid complications, we shall assume that $X$ is a Banach space. For any subset $D$ of $X$, we let

$$
\mathcal{L}_{I}(D)=\{\gamma \in C(X, X) ; I \circ \gamma \leq I, \gamma(D) \subseteq D \text { and } \gamma(x)=x \text { on } X \backslash D\},
$$

where $I$ is a given functional on $X$. Recall that the topological dimension (or covering dimension) of a metric space $D$ (in short, topdim $D$ ) is the least integer $m$ such that any finite open covering $\mathcal{O}$ of $D$ can be refined by an open covering $\mathcal{O}_{1}$ such that any $x \in D$ belongs to at most $m+1$ elements of $\mathcal{O}_{1}$.

Definition 9.2. Let $I$ be a continuous functional on $X$ and let $x$ be a point in $K_{c}$, the critical set at level $c$.

(1) Define $\operatorname{sad}(x)$ to be the least integer $k$ for which there is a neighborhood $N$ of $x$ with the following property: for any sub-neighborhood $M \subset N$ of $x$, the set $M \cap I^{c}$ is not $(k-1)$-connected.

(2) Define $\operatorname{sad}^{*}(x)$ to be the least integer $k$ such that for any neighborhood $N$ of $x$, there exists a sub-neighborhood $M \subset N$ of $x$ and $\gamma$ in $\mathcal{L}_{I}(N)$ with topdim $\gamma(M) \leq k$.

(3) For a point $x$ in $K_{c}$, define $\operatorname{Ord}(x)$ to be the set of all integers $k \geq 1$ for which there is a neighborhood $N$ of $x$ with the following property: for any open sub-neighborhood $M \subset N$ of $x$ with $H_{k}(M)=0$, the homology group $H_{k-1}\left(I^{c} \cap M\right)$ is not trivial.

If we agree to say that a set is -1-connected if it is non-empty, then $x$ is a minimum if and only if $\operatorname{sad}(x)=0$. Also, a critical point $x$ of mountain-pass type is a critical point with $\operatorname{sad}(x)=1$. It is clear that -in the smooth setting- these indices are closely related to the Morse indices of the critical points.

The following is a particular case of a result of Fang $[\mathrm{F}]$. We consider the case where $K_{c}$ consists of only isolated points. Otherwise, one needs to alter slightly the definitions and settle for statements on subsets of - as opposed to points in $-K_{c}$.

Theorem 9.2. Let $I$ be a continuous functional on $X$ and let $\mathcal{F}$ be a homotopy stable family with boundary $B$. Assume that $I$ verifies $(P S)_{c}$ where $c:=c(I, \mathcal{F})$, that $\sup _{B} I<c$ and that $K_{c}$ consists of isolated critical points.

(1) If $\mathcal{F}$ is homotopic of dimension $n$, then there is $x \in K_{c}$ with $\operatorname{sad}(x) \leq n$. 
(2) If $\mathcal{F}$ is cohomotopic of dimension $n$, then there is $x \in K_{c}$ with $\operatorname{sad}(x) \geq n$.

(3) If $\mathcal{F}$ is homological of dimension $n$, then there is an $x \in K_{c}$ with $n \in \operatorname{Ord}(x)$.

\section{Elements OF SYMPLECTIC TOPOLOGY}

In this last example, we show how the study of critical points leads to new invariants in symplectic geometry. A crucial property of these invariants is monotonicity (with respect to inclusion), which they inherit from the min-max procedure.

Consider one last time the symplectic space $\left(\mathbf{R}^{2 n}, J\right)$. A differentiable map $\varphi$ from an open subset $\Omega$ of $\mathbf{R}^{2 n}$ into itself is symplectic if its tangent map at every point preserves the symplectic structure: $\left(J \varphi^{\prime}(x) \xi, \varphi^{\prime}(x) \xi\right)=(J \xi, \xi)$ for all $(x, \xi) \in \Omega \times \mathbf{R}^{2 n}$.

A symplectic capacity is a function which associates to every subset $A$ of $\mathbf{R}^{2 n}$ an extended real number $c(B) \geq 0$ with the following properties:

- (Monotonicity) If $A \subset B$, then $c(A) \leq c(B)$;

- (Invariance) If $\varphi$ is a symplectic map, then $c(\varphi(A))=c(A)$ for all $A$;

- (2-Homogeneity) $c(\lambda A)=\lambda^{2} c(A)$ for all $\lambda \in \mathbf{R}$.

Note that if $n>1$, the volume is not a symplectic capacity because of the last condition. The first nontrivial symplectic capacity was discovered by Gromov, with spectacular implications, and many others have been discovered since. We will follow the approach of Ekeland-Hofer [E-H3].

Denote by $\omega$ the differential two-form on $\mathbf{R}^{2 n}$ defined by $\omega_{x}(\xi, \eta)=(J \xi, \eta)$ for all $x \in \mathbf{R}^{2 n}, \xi, \eta \in T_{x} \mathbf{R}^{2 n}$. Note that $\omega=d \Omega$, where $\Omega_{x}(\xi)=(J x, \xi)$. Given a smooth, compact hypersurface $\Sigma \subset \mathbf{R}^{2 n}$, denote by $i: \Sigma \mapsto \mathbf{R}^{2 n}$ the imbedding, and by $i^{\star} \omega$ the pullback of $\omega$ on $\Sigma$. Say that $\Sigma$ is of contact type if there is a non-vanishing one-form $\Theta$ on $\Sigma$ such that $i^{\star} \omega=d \Theta$, and $\Theta$ does not vanish on the one-dimensional space $\operatorname{Ker}\left(i^{\star} \omega\right)$. Every convex hypersurface and, more generally, every $\Sigma$ which is star-shaped around some point (which may be assumed to be the origin) are of contact type. Indeed, $\Theta:=i^{\star} \Omega$ will do the job.

Choose a basis in $\mathbf{R}^{2 n}$ where $(J x, y)=\sum_{i=1}^{n}\left(x_{i} y_{i+n}-x_{i+n} y_{i}\right)$. Denote by $B$ the unit ball in $\mathbf{R}^{2 n}$ and by $D$ the cylinder $\left\{x ; x_{1}^{2}+y_{1}^{2} \leq 1\right\}$. Finally, define the action of any loop $x(t)$ in $\mathbf{R}^{2 n}$ by $A(x)=\frac{1}{2} \oint(J x, d x)$.

Theorem 10.1. There is a symplectic capacity $c$ on $\mathbf{R}^{2 n}$ such that $c(B)=c(D)=$ $\pi$. If an open subset $U$ is bounded by a smooth compact hypersurface of contact type, then there is a closed symplectic trajectory $x$ on its boundary $\partial U$ such that $c(U)=k A(x)$ for some integer $k \geq 1$.

The proof starts with investigating periodic solutions of a special class of Hamiltonian systems. With any $C^{2}$-function $H$ in $\mathbf{R}^{2 n}$, associate the boundary-value problem

$$
\begin{cases}\dot{x} & =J H^{\prime}(x) \\ x(0) & =x(1)\end{cases}
$$

and consider the functional $I_{H}(x)=A(x)-\int_{0}^{1} H(x(t)) d t$ on the Hilbert space $X=$ $H^{1 / 2}\left(S^{1} ; \mathbf{R}^{2 n}\right)$. More than 150 years ago, Hamilton showed that the critical points of $I_{H}$ over $C^{1}\left(S^{1} ; \mathbf{R}^{2 n}\right)$ solve problem (10.1). It still holds true in $H^{1 / 2}\left(S^{1} ; \mathbf{R}^{2 n}\right)$, although there is a technical difficulty because elements of this space need not be continuous functions. 
Lemma 10.1. Assume that $H$ is $C^{2}$ and non-negative on $\mathbf{R}^{2 n}$, that it vanishes in a neighbourhood $U$ of the origin, and that there are constants $R>0, C>\pi$ and $1=\alpha_{1} \geq \ldots \geq \alpha_{n}>0$, such that $H(x) \geq C \sum_{i=1}^{n} \alpha_{i}\left(x_{i}^{2}+x_{i+n}^{2}\right)$ for all $|x|>R$. Then the function $I_{H}$ has a critical point $\bar{x}$ satisfying $I_{H}(\bar{x})>0$.

The lemma is proved by setting up an appropriate gradient-stable class of sets. Note that, because of the assumptions on $H$, every critical point $x_{0}$ of $H$ in $\mathbf{R}^{2 n}$, for instance every point in $U$, yields a critical point $x(t)=x_{0}$ of $I_{H}$ on $X$ with $I_{H}(x)<0$. The estimate $I_{H}(\bar{x})>0$ is therefore crucial in finding non-trivial solutions.

Write an orthogonal splitting $X=X^{-} \oplus X^{0} \oplus X^{+}$, where $X^{-}$(resp. $\left.X^{+}\right)$ consists of those $x$ whose Fourier coefficients $x_{k}$ vanish for $k>0$ (resp. $k<0$ ), and $X^{0}=\mathbf{R}^{2 n}$ are just the constants. We then have $A(x)=\frac{1}{2}\left\|x^{+}\right\|^{2}-\frac{1}{2}\left\|x^{-}\right\|^{2}$. Define $e_{1}^{+} \in X^{+}$by $e_{1}^{+}(t)=e^{2 \pi J t} x_{0}$, and consider the following subsets $\Sigma$ and $\Gamma$ of $X$ :

$$
\begin{aligned}
\Sigma & =\left\{x=s e_{1}^{+}+x^{0}+x^{-} ;\left\|x^{0}+x^{-}\right\| \leq R \text { and } 0 \leq s \leq R\right\} \\
\Gamma & =\left\{x \in X^{+} ;\|x\|=r\right\}
\end{aligned}
$$

where $r>0$ and $R>0$. Using the assumptions on $H$ (in particular the fact that $C>\pi$ ), one shows that $R$ can be chosen so large that $I_{H}(x) \leq 0$ for all $x \in \partial \Sigma$, and that $r>0$ can be chosen so small that for some $\beta>0$ we have $I_{H}(x) \geq \beta$ for all $x \in \partial \Gamma$. We then consider the gradient flow $\varphi_{t}$ on $X$, associated with the equation $\dot{x}=-I_{H}^{\prime}(x)$. It is well-defined because $I_{H}^{\prime}(x)$ is Lipschitz, as a consequence of the assumptions on $H$. Moreover, we have $I_{H}^{\prime}(x)=x^{+}-x^{-}-j H^{\prime}(x)$, where $j: H^{-1 / 2} \mapsto H^{1 / 2}$ is the compact operator defined -by way of Fourier series- by $j\left(\Sigma_{n} e^{2 \pi J n t} x_{n}\right)=\Sigma_{n} \frac{1}{\max (1,|n|)} e^{2 \pi J n t} x_{n}$. The map $\varphi_{t}$ can be decomposed as the sum of an isomorphism and a (non-linear) compact map. Since $I_{H}$ is non-positive on $\partial \Sigma$ and decreases on the gradient lines, we have $I_{H}\left(\varphi_{t}(\partial \Sigma)\right) \leq 0$, so that $\varphi_{t}(\partial \Sigma) \cap \Gamma=\emptyset$. Using this fact, and applying Leray-Schauder degree theory, one shows that:

$$
\varphi_{t}(\Sigma) \cap \Gamma \neq \emptyset, \text { for all } t \text {. }
$$

In other words, $\Gamma$ is dual to the family $\mathcal{F}=\left\{\varphi_{t}(\Sigma) \mid t \geq 0\right\}$. Consider the inf-sup value $c\left(I_{H}, \mathcal{F}\right)$. It is seen to be finite, and because of duality:

$$
0<\beta \leq \inf _{\Gamma} I_{H}(x) \leq \sup _{\varphi_{t}(\Sigma)} I_{H}(x) \leq c(I, \mathcal{F})
$$

A further argument shows that $I_{H}$ satisfies the Palais-Smale condition. It follows that $c\left(I_{H}, \mathcal{F}\right)$ is a critical value. Since it is positive, the corresponding periodic solution is non-constant.

We now proceed to the proof of Theorem 10.1. Given any subset $U$ of $\mathbf{R}^{2 n}$, define $\mathcal{H}(U)$ to be the set of all Hamiltonians $H$ satisfying the assumptions of Lemma 10.1 and vanishing in a neighbourhood of $\bar{U}$, the closure of $U$. We then define $c(U)$, the symplectic capacity of $U$, by the formula:

$$
c(U)=\inf _{H \in \mathcal{H}(U)} c\left(I_{H}, \mathcal{F}\right)
$$

It is clear that $c$ is monotonic: if $U \subset V$, then $\mathcal{H}(V) \subset \mathcal{H}(U)$ and $c(U) \leq c(V)$. It also follows from the definition that $c$ is non-negative, is homogeneous of degree two, and is invariant under symplectic tranformations. To show that it is not identically zero, take a sequence of Hamiltonians $H_{n} \in \mathcal{H}(U)$ such that $c_{n}:=c\left(I_{H_{n}}, \mathcal{F}\right) \rightarrow$ $c(U)$. For each of them, there is a periodic solution $x_{n}(t)$ of the corresponding 
Hamiltonian system, and $A\left(x_{n}\right)-\int_{0}^{1} H_{n}\left(x_{n}(t)\right) d t=c_{n}$. A detailed analysis shows that $H_{n}\left(x_{n}(t)\right)$, which is constant in $t$, goes to zero, so that the trajectories $x_{n}$ converge to $\bar{U}$ in the Hausdorff topology, and $A\left(x_{n}\right) \rightarrow c(U)$. Since $\dot{x}_{n}=J H_{n}^{\prime}\left(x_{n}\right)$ and $H_{n}$ vanishes on $U$, we have that $x_{n}(t) \notin U$ for all $t$, so that $x_{n}$ converges to $\partial U$.

If $\partial U$ is of contact type, pleasant things happen. First of all, we can foliate a neighbourhood of $\partial U$ by symplectic images of $\partial U$, and choose the Hamiltonians $H_{n}$ so that their level sets $H_{n}=h$, for small enough $h$, are precisely the leaves of the foliation. Then any periodic solution of $\dot{x}=J H_{n}^{\prime}(x)$, lying on one of these energy levels, is the image of a closed trajectory of the symplectic flow on $\partial U$. As $n \rightarrow \infty$ and $H_{n}\left(x_{n}\right) \rightarrow 0$, we eventually enter the foliated neighbourhood, and we get a sequence of closed trajectories $y_{n}$ of the symplectic flow on $\partial U$ and integers $k_{n}$ such that $k_{n} A\left(x_{n}\right) \rightarrow c(U)$, the presence of the $k_{n}$ paying due regard to the fact that $y_{n}$ may be run around several times.

Next, since $\partial U$ is of contact type, we have $(J x, \dot{x}) \geq a|\dot{x}|$ for some constant $a>0$ depending only on $\partial U$. It follows that $A\left(y_{n}\right) \geq a$ Length $\left(y_{n}\right)$, so the lengths of the $y_{n}$ are uniformly bounded, and since they all are trajectories of the symplectic flow on $\partial U$, the Ascoli theorem implies that they converge $C^{1}$ to some $x(t)$, which also is a closed trajectory of the symplectic flow on $\partial U$. We have $c(U)=k A(y)>0$ for some $k \geq 1$. In the cases of the ball $B$ and the cylinder $D$, one can show that $k=1$, and $A(x)=\pi$.

\section{BY WAY OF CONCLUSION}

There have been other quite successful -and more global- ways to study critical point theory and its ramifications. There is the point of view taken by Taubes in his study of the Yang-Mills equations [Tau3] where he develops a Morse theory for the Yang-Mills functional $I_{Y M}$ even though it fails the Palais-Smale condition. Taubes considers what he calls the "relevant non-compact ends" of the Banach manifold (i.e., the sets of $\epsilon$-critical points around level $c$ ) and analyzes the functional on these sets. There are special values of the energy $c$ where the behaviour of $I_{Y M}$ restricted to $K_{c}(\epsilon)$ has yielded to analysis. For example, in one of the amenable cases (where $c \leq|k|+2, k$ being the first Pontrjagin class of the associated vector bundle) Taubes uses this approach to prove that the moduli spaces of self-dual connections on $S^{4}$ are path connected. We refer to the references in [Tau3] for a list of related work by Taubes and others.

A closely related approach has been developed by Bahri Ba and his collaborators where a related analysis is done via the notion of "critical points at infinity" (essentially those elements in $K_{c}(\epsilon)$ that lie on negative-gradient flows). Roughly speaking, these objects can sometimes be described for $\epsilon$ small enough as $\delta(\epsilon)$ neighborhoods (in an appropriate metric) of the union $K \cup W$ of two sets: $K$ being a set of true critical points in $I^{c+\epsilon} \backslash I^{c-\epsilon}$ while $W$ (a set of virtual critical points!) is a finite dimensional spinal set that is parametrizable by a finite dimensional space of data. The idea is to show that the topology of $W$ alone does not account for the change in topology between $I^{c+\epsilon}$ and $I^{c-\epsilon}$ and therefore some true critical points exist.

This type of description is not easy and has been a recurrent theme in borderline variational problems. But this formidable task has been accomplished in many 
important cases: Saks-Uhlenbeck for harmonic maps [S-U], Bahri-Coron on semilinear elliptic equations involving the critical Sobolev exponent Ba-Co2, BahriRabinowitz [Ba-Ra] on the 3-body problem, as well as the work of Taubes mentioned above.

There are also the infinite dimensional extensions of R. Thom's investigation of the so-called Morse complex (consisting of all the paths of the negative-gradient flow connecting one critical point to another at a lower level). The work of Milnor, Smale, Witten and others has been extended to some important infinite dimensional settings by Floer. This approach has been successful in the study of periodic trajectories for the symplectic flow on a compact symplectic manifold, where the negative gradient flow for the action functional reduces to the Cauchy-Riemann equations. This is an ongoing program which was initiated by A. Floer [Fl, with spectacular results [HTWZ], and this is where we stop.

\section{REFERENCES}

[A-R] A. Ambrosetti, P. H. Rabinowitz: Dual variational methods in critical point theory and applications. J. Funct. Anal. 14 (1973), 349-381. MR 51:6412

[A-CZ] A. Ambrosetti, V. Coti Zelati, Periodic solutions of singular Lagrangian systems, Birkhauser. Boston, Basel, Berlin (1993). MR 95b:58054

[Au] T. Aubin: Nonlinear analysis on manifolds. Monge-Ampère equations, Grundlehren 252, Springer, New York-Heidelberg-Berlin (1982). MR 85j:58002

[Ba] A. Bahri: Critical points at infinity in some variational problems, Pitman Research Notes Math. 182, Longman House, Harlow (1989). MR 91h:58022

[Ba-Be] A. Bahri, H. Berestycki: A perturbation method in critical point theory and applications T.A.M.S. 267 (1981) 1-32. MR 82j:35059

[Ba-Co1] A. Bahri, J. M. Coron: The scalar-curvature problem on the standard threedimensional sphere, J. Funct. Anal. 95 (1991) 106-172. MR 92k:58055

[Ba-Co2] A. Bahri, J. M. Coron: On a nonlinear elliptic equation involving the critical Sobolev exponent: the effect of the topology of the domain, Comm. Pure Appl. Math. 41 (1988), 253-294. MR 89c:35053

[Ba-L1] A. Bahri, P. L. Lions: Morse index of some min-max critical points I. Application to multiplicity results. Comm. Pure \& App. Math. 41 (1988), 1027-1037. MR 90b:58035

[Ba-L2] A. Bahri, P. L. Lions: Solutions of superlinear elliptic equations and their Morse indices, Comm. Pure Appl. Math. XLV (1992), 1205-1215. MR 93m:35077]

[Ba-Ra] A. Bahri, P. Rabinowitz: Periodic Solutions of Hamiltonian Systems of three-body type, A.I.H.P., Analyse nonlinéaire (1991), 561-649. MR 92k:58223

[B-R] V. Benci, P. H. Rabinowitz: Critical point theorems for indefinite functionals, Invent. Math., 52 (1979), 241-273. MR 80i:58019

[Be-L] H. Berestycki, P. L. Lions: Nonlinear scalar field equations, Part I, Arch. Rat. Mech. Anal., 82 (1983), 313-346. Part II 82 (1983), 347-376. MR 84h:35054a

[Bo] P. Bolle: On the Bolza problem, J. Diff. Equations, 152 (2) (1999), 274-288. MR 99m:58043

[B-G-T] P. Bolle, N. Ghoussoub, H. Tehrani: The Multiplicity of solutions in non-homogenous boundary value problems, Manuscripta Matematica, 101 (2000), 325-350. MR 2001c:58010

[B-N] H. Brezis and L. Nirenberg: Positive solution of nonlinear elliptic equations involving critical Sobolev exponents, Comm. Pure Appl. Math. 36 (1983), 437-477. MR 84h:35059

[B-C] H. Brezis and J. M. Coron: Multiple solutions of H-systems and Rellich's conjecture, Comm. Pure App. Math. 37 (1984), 149-187. MR 85i:53010

[C-K-N] L. Caffarelli, R. Kohn, L. Nirenberg: First Order Interpolation Inequality with Weights, Compositio Math. 53 (1984), 259-275. MR 86c:46028

[C-G] C. Chambers and N. Ghoussoub: Deformation from symmetry and multiplicity of solutions in non-homogeneous problems, Journal of Discrete and Continuous Dynamical Systems 8, No. 1 (2002), 267-281. 
[Ch] K. C. Chang: Infinite dimensional Morse theory and multiple solution problems, Progress in nonlinear diff. equations, Birkhäuser, Boston (1993). MR 94e:58023

[Co1] C. V. Coffman: Ljusternik-Schnirelman theory: Complementary principles and the Morse index. Nonlinear Analysis, Theory \& Applications. Vol 12, No. 5 (1988), 507529. MR 89g:58037

[Co2] C. V. Coffman: A nonlinear boundary value problem with many positive solutions, Journal of Diff. Equations, 54 (3) (1984), 429-437. MR 86e:35055

[Con] C. Conley: Isolated invariant sets and the Morse index, CBMS 38, AMS, Providence (1978). MR 80c:58009

[C-D-M] J.N. Corvellec, M. Degiovanni, M. Marzocchi: Deformation properties for continuous functionals and critical point theory, Top. Meth. Nonl. Anal. 1 (1993) 151-171. MR 94c:58026

[C-E-L] V. Coti Zelati, I. Ekeland, P.L. Lions: Index estimates and critical points of functionals not satisfying Palais-Smale, Annali della Scuola Normale Superiore di Pisa, Scienze Fisiche e Matematiche, Serie IV, Vol XVII, Fasc.4 (1990), 569-582. MR 92a:58027

[C-R1] V. Coti Zelati, P.H. Rabinowitz, Homoclinic orbits for second order Hamiltonian systems possessing superquadratic potentials, J. Amer. Math. Soc. 4 (1991), 693-727. MR 93e:58023

[C-R2] V. Coti Zelati and P.H. Rabinowitz, Homoclinic type solutions for a semilinear elliptic PDE on $R^{n}$, Comm. Pure Appl. Math. 45 (1992), 1217-1269. MR 93k:35087

[D-J-L-W] W. Ding, Y. Yost, J. Li, X. Wang, Existence results for mean field equations, AIHP, 16, 5 (1999), 653-666. MR 2000i:35061

[D-F] H. Donnelly and C. Fefferman, Nodal sets of eigenfunctions on Riemannian manifolds, Invent. Math. 93 (1988), 161-183. MR 89m:58207

[Eg] H. Egnell: Positive Solutions of Semilinear Equations in Cones, Trans. AMS 330, 1 (1992), 191-201. MR 92f:35018

[E0] I. Ekeland: Une theorie de Morse pour les systèmes Hamiltoniens convexes, Annales de l'IHP, Analyse non linéaire, 1 (1984), 19-78. MR 85f:58023

[E1] I. Ekeland: Nonconvex minimization problems, Bull. AMS 1 (1979), 443-474.

[E2] I. Ekeland: Convexity Methods in Hamiltonian Mechanics. Springer-Verlag, Berlin, Heidelberg, New-York (1990). MR 91f:58027

[E-H1] I. Ekeland, H. Hofer: Periodic solutions with prescribed period for convex autonomous Hamiltonian systems. Inv. Math. 81 (1985), 155-188. MR 87b:58028

[E-H2] I. Ekeland, H. Hofer: Convex Hamiltonian energy surfaces and their periodic trajectories. Commun. Math. Phys. 113 (1987), 419-469. MR 89c:58035

[E-H3] I. Ekeland, H. Hofer: Symplectic topology and Hamiltonian dynamics I and II, Math. Zeitschrift 200 (1989), 355-378 and 203 (1990), 553-567. MR 90a:58046, MR 91e:58053

[E-L] I. Ekeland, J.M. Lasry: On the number of periodic trajectories for a Hamiltonian flow on a convex energy surface. Ann. of Math. 112 (1980), 283-319. MR 81m:58032

[E-S] M. Esteban, E. Séré: Solutions of the Dirac-Fock Equations for Atoms and Molecules, Commun. Math. Phys. 203 (1999), 499-530. MR 2000j:81057

[F-H-R] E. Fadell, S. Husseini, P.H. Rabinowitz: Borsuk-Ulam theorems for arbitrary $S^{1}$ actions and applications. T.A.M.S. 274 (1982), 345-360. MR 84b:55001

[F] G. Fang: On the existence and the classification of critical points for non-smooth functionals, Canadian Journal of Mathematics, Vol. 47 (1995), 684-717. MR 96j:58029

[F-G] G. Fang, N. Ghoussoub: Morse-type information on Palais-Smale sequences obtained by Min-Max principles. Comm. Pure \& Applied Math., XLVII, (1994), 1595-1653. MR 95m:58028

[Fl] A. Floer: Witten's complex and infinite dimensional Morse theory, J. Diff. Geom. 30 (1989), 207-221. MR 90d:58029

[G1] N. Ghoussoub: Location, multiplicity and Morse Indices of min-max critical points, J. Reine. und. angew. Math. 417 (1991), 27-76. MR 92e:58040

[G2] N. Ghoussoub: Duality and Perturbation Methods in Critical Point Theory, Cambridge Tracts in Mathematics, Cambridge University Press (1993). MR 95a:58021

[G3] N. Ghoussoub: Morse theory up to epsilon, Preprint (2000).

[G-K] N. Ghoussoub, X. Kang: Semilinear equations with Hardy-Sobolev singularities on the boundary, Preprint (2001). 
[G-P] N. Ghoussoub, D. Preiss: A general mountain pass principle for locating and classifying critical points. A.I.H.P-Analyse non linéaire, 6, No. 5 (1989), 321-330. MR 91a:58043

[G-Y] N. Ghoussoub, C. Yuan: Multiple Solutions for Quasi-linear PDEs Involving the Critical Sobolev and Hardy Exponents, Trans. A.M.S, 352, 12 (2000) 5703-5743. MR 2001b:35109

[G-N-N] B. Gidas, W.-M. Ni, L. Nirenberg: Symmetry of positive solutions of nonlinear elliptic equations in $R^{n}$, Advances in Math. Supplementary Studies, 7A, Academic, New York (1981) 369-402. MR 84a:35083

[G-G] C. Gui, N. Ghoussoub: Multi-peak solutions for a semilinear Neumann problem with critical exponent, Math. Z. 229 (1998), 443-474. MR 2000k:35097

[Gu1] C. Gui: Existence of multi-bump solutions for nonlinear Schrödinger equations via variational method, Comm. Partial Diff. Eq., 21 (1996), 787-820. MR 98a:35122

[Gu2] C. Gui: Multi-peak solutions for a semilinear Neumann problem, Duke Math. J. 84 (1996), 739-769. MR 97i:35052

[H-S] R. Hardt and L. Simon: Nodal sets for solutions of elliptic equations, J. Differential Geom. 30 (1989), 505-522. MR 90m:58031

[H] H. Hofer: A geometric description of the neighborhood of a critical point given by the mountain pass theorem, J. London Math. Soc., 31 (1985), 566-570. MR 87e:58041

[HTWZ] H. Hofer, C. Taubes, A. Weinstein, E. Zehnder: Floer memorial volume, Birkhaüser, 1994. MR 96f:58001

[J] L. Jeanjean: On the existence of bounded Palais-Smale sequences and application to a Landesman-Lazer type problem set on $\boldsymbol{R}^{N}$, Proc. Roy. Soc. Edinburgh, 129A (1999), 787-809. MR 2001c:35034

[J-K] D. Jerison, C. Kenig: Unique continuation and absence of positive eigenvalues for Schrödinger operators, Ann. Math. 121 (1985), 463-494. MR 87a:35058

[L-S] A. Lazer, S. Solimini: Nontrivial solutions of operator equations and Morse indices of critical points of Min-Max type. Non Linear Analysis, Methods \& Applications,12, No. 8 (1988) 761-775. MR 89i:58018

[Lieb] E. H. Lieb: Sharp Constants in the Hardy-Littlewood-Sobolev and Related Inequalities, Ann. of Math. 118 (1983), 349-374. MR 86i:42010

[L1] P. L. Lions: The concentration-compactness principle in the calculus of variations. The locally compact case. Part I and II., Ann. Inst. H. Poincaré, Anal. non-lin., 1 (1984), 109-145 and 223-283. MR 87e:49035a, MR 87e:49035b

[L2] P. L. Lions: Solutions of Hartree-Fock equations for Coulomb systems. Comm. Math. Physics, 109 (1987), 33-97. MR 88e:35170

[L-L-Z] C-G. Liu, Y. Long, C. Zhu: Multiplicity of closed characteristics on symmetric convex hypersurfaces in $\mathbf{R}^{2 n}$, Nankai Inst. Math., Preprint (1999).

[L-Sc] L. Ljusternik, L. Schnirelmann: Méthodes topologiques dans les problèmes variationels. Hermann, Paris (1934).

[Lo1] Y. Long: Bott formula of the Maslow-type index theory, Pacific J. of Math., 187 (1999), 113-143 MR 2000d:37073

[Lo2] Y. Long: Precise iteration formulae of the Maslow-type index theory and ellipticity of closed characteristics, Advances in Math., 154 (2000), 76-131. MR 2001j:37111

[L-Z] Y. Long, C. Zhu: Closed characteristics on compact convex hypersurfaces in $\mathbf{R}^{2 n}$, Nankai Inst. Math., Preprint (1999).

[M-W] J. Mawhin, M. Willem: Critical point theory and Hamiltonian systems. Applied Mathematical Sciences, 74, Springer Verlag (1989). MR 90e:58016

[N-T2] W. -M. Ni, I. Takagi, Locating the peaks of least energy solutions to a semilinear Neumann problem, Duke Math. J. 70 (1993), 247-281. MR 94h:35072

[N] L. Nirenberg: Variational Methods in non-linear problems. Lecture notes in Math, 1365, Springer-Verlag (1989), 100-119. MR 90c:58034

[P] S. Pohozaev: Eigenfunctions of the equation $\Delta u+\lambda f(u)=0$, Soviet Math. Dokl., 6, (1965), 1408-1411. MR 33:411.

[P-S] P. Pucci, J. Serrin: The structure of the critical set in the mountain pass theorem. T.A.M.S. 299, No. 1 (1987), 115-132. MR 88b:58025

[R1] P. H. Rabinowitz: Minimax methods in critical point theory with applications to differential equations. C.B.M.S., A.M.S., No. 65 (1986). MR 87j:58024 
[R2] P. Rabinowitz, Multibump solutions of differential equations: an overview, Chinese Journal of Mathematics, 24, 1 (1996), 1-36. MR 97h:58084

[R3] P. Rabinowitz: Periodic solutions of a Hamiltonian system on a prescribed energy surface. J. Diff. Eq. 33 (1979), 336-352. MR 81a:58030a

[R4] P. Rabinowitz: Periodic solutions of Hamiltonian systems. Comm. Pure App. Math. 31 (1978), 157-184. MR 57:7674]

[S-U] J. Sacks, K. Uhlenbeck: On the existence of minimal immersions of 2-spheres. Ann. of Math. 113 (1981), 1-24. MR 82f:58035

[Se] E. Séré, Existence of infinitely many homoclinic orbits in Hamiltonian systems, Math. Z. 209 (1992), 27-42. MR 92k:58201

[So] S. Solimini: Morse index estimates in Min-Max theorems. Manuscripta Mathematica, 63 (1989), 421-433. MR 90f:58028

[St] M. Struwe: Variational methods and their applications to non-linear partial differential equations and Hamiltonian systems. Springer-Verlag (1990). MR 92b:49002

[St1] M. Struwe: The existence of surfaces of constant mean curvature with free boundaries, Acta Math. 160 (1988), 19-64. MR 89a:53012

[St-Ta] M. Struwe, G. Tarantello: On multivortex solutions in Chern-Simons gauge theory, Bollettino U.M.I. 1 (1998), 109-121. MR 99c:58041

[Su] T. Suzuki: Positive solutions for semilinear elliptic equations on expanding annuli: mountain pass approach, Funkcial. Ekvac. 39, 1 (1996), 143-164. MR 97e:35065

[Tan1] K. Tanaka: Morse indices at critical points related to the symmetric mountain pass theorem and applications, Comm. in part. diff. equations, 14 (1), (1989), 99-128. MR 90i:58019

[Tan2] K. Tanaka: Periodic solutions for singular Hamiltonian systems and closed geodesics on non-compact Riemannian manifolds, AIHP, Analyse non-linéaire, 17, 1 (2000) 1-33. MR 2001e:37081

[Tar] G. Tarantello: Nodal Solutions of Semi-linear Elliptic Equations with Critical Exponent, Differential and Integral Equations, 5, No 1 (1992), 25-42. MR 92k:35109

[Tau1] C. H. Taubes: Path-Connected Yang-Mills Moduli Spaces, J. Differential Geometry, 19 (1984), 337-392. MR 85m:58049

[Tau2] C. H. Taubes: Min-Max Theory for the Yang-Mills-Higgs Equations, Commun. Math. Phys. 97 (1985), 473-540. MR 86m:58042

[Tau3] C. H. Taubes: A framework for Morse theory for the Yang-Mills functional, Invent. Math. 94 (1988), 327-402. MR 90a:58035

[V] C. Viterbo: Indice de Morse des points critiques obtenus par minimax, A.I.H.P. Analyse non linéaire, 5, No. 3 (1988), 221-225. MR 89k:58055

[Ya] Xue-Fang Yang: Nodal sets and Morse indices of solutions of super-linear elliptic equations, J. Funct. Analysis 160, 1 (1998), 223-253. MR 99j:35058

CEREmade, Université Paris-Dauphine, Paris, France

E-mail address: Ivar.Ekeland@dauphine.fr

Pacific Institute for the Mathematical Sciences, University of British Columbia, Vancouver, B.C., CAnada V6T 1 Z2

E-mail address: nassif@math.ubc.ca 